\title{
Efficient Collaborations in Supply Chains
}

Citation for published version (APA):

Jung, V. (2020). Efficient Collaborations in Supply Chains. [Doctoral Thesis, Maastricht University, Universiteit Hasselt]. ProefschriftMaken. https://doi.org/10.26481/dis.20200520vj

Document status and date:

Published: 01/01/2020

DOI:

10.26481/dis.20200520vj

Document Version:

Publisher's PDF, also known as Version of record

\section{Please check the document version of this publication:}

- A submitted manuscript is the version of the article upon submission and before peer-review. There can be important differences between the submitted version and the official published version of record.

People interested in the research are advised to contact the author for the final version of the publication, or visit the DOI to the publisher's website.

- The final author version and the galley proof are versions of the publication after peer review.

- The final published version features the final layout of the paper including the volume, issue and page numbers.

Link to publication

\footnotetext{
General rights rights.

- You may freely distribute the URL identifying the publication in the public portal. please follow below link for the End User Agreement:

www.umlib.nl/taverne-license

Take down policy

If you believe that this document breaches copyright please contact us at:

repository@maastrichtuniversity.nl

providing details and we will investigate your claim.
}

Copyright and moral rights for the publications made accessible in the public portal are retained by the authors and/or other copyright owners and it is a condition of accessing publications that users recognise and abide by the legal requirements associated with these

- Users may download and print one copy of any publication from the public portal for the purpose of private study or research.

- You may not further distribute the material or use it for any profit-making activity or commercial gain

If the publication is distributed under the terms of Article $25 \mathrm{fa}$ of the Dutch Copyright Act, indicated by the "Taverne" license above, 
Doctoral dissertation submitted to obtain the degree of Doctor at Maastricht University and Doctor of Business Economics at UHasselt, to be defended by

\section{Verena Jung}

\section{Efficient Collaborations in Supply Chains}

\section{Maastricht University}

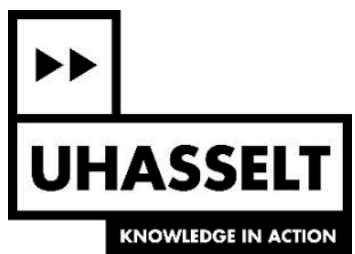

Supervisor:

Prof. dr. Alexander Grigoriev

Co-supervisor:

Dr. Tjark Vredeveld
Supervisor:

Prof. dr. Katrien Ramaekers

Co-supervisor:

Dr. Lotte Verdonck 



\title{
Efficient Collaborations in Supply Chains
}

\author{
Verena Jung
}


(C) Verena Jung, Maastricht 2020.

All rights reserved. No part of this publication may be reproduced, stored in a retrieval system, or transmitted in any form, or by any means, electronic, mechanical, photocopying, recording or otherwise, without the prior permission in writing from the author.

This book was typeset by the author using $\mathrm{LAT}_{\mathrm{E} X}$.

Published by ProefschriftMaken; www.proefschriftmaken.nl ISBN: 978-94-6380-825-5

D/2020/2451/20

Printed in The Netherlands by ProefschriftMaken; www.proefschriftmaken.nl 


\section{EFFICIENT COLLABORATIONS IN SUPPLY CHAINS DISSERTATION}

to obtain the degree of Doctor at the Maastricht University, on the authority of the Rector Magnificus, Prof.dr. Rianne M. Letschert in accordance with the decision of the Board of Deans, to be defended in public on Wednesday 20 May 2020 at 10.00 hours by

Verena Jung 


\title{
Supervisors:
}

Prof. dr. A. Grigoriev

Prof. dr. K. Ramaekers (UHasselt)

\section{Co-supervisors:}

Dr. T. Vredeveld

Dr. L. Verdonck (UHasselt)

\author{
Assessment Committee: \\ Prof. dr. ir. C.P.M. van Hoesel (chair) \\ Prof. dr. A. Caris (UHasselt) \\ Prof. dr. A. Abiad Monge (TU Eindhoven) \\ Prof. dr. K. Sörensen (Universiteit Antwerpen)
}

This research was financially supported by COMEX (Combinatorial Optimization: Metaheuristics \& Exact Methods). 
To my parents and my boyfriend, for their invaluable support 



\section{Acknowledgements}

My years as a $\mathrm{PhD}$ student have been one of the toughest but at the same time the most exciting, interesting and instructive years in my life. I am really happy that I took this journey, since I have not only improved as a researcher and teacher, but I have grown a lot as a person. This would not have been possible without the enormous help and support of many people whom I would like to thank in the following.

First of all, I would like to express my gratitude to my promoters and copromoters: Alexander Grigoriev and Tjark Vredeveld from Maastricht University as well as Katrien Ramaekers and Lotte Verdonck from UHasselt. Thank you for providing me the opportunity to do the joint $\mathrm{PhD}$ program. The cooperation between Maastricht and Hasselt provided me with a lot of new insights and significantly improved my research. Alex, thank you for providing me with many nice and interesting research topics and for sharing your inspiring ideas with me. I always knew that I am welcome in your office. Tjark, thank you for your great support and help during the three years. You always took your time to give me advise and to help me to improve my research. I am really grateful for that. Katrien, thank you for always warmly welcome me at UHasselt and for taking your time to thoroughly read this thesis. Lotte, I am so grateful that you shared your experiences and knowledge with me. Thank you for helping me to raise this work to a higher level and for supporting me so much during my $\mathrm{PhD}$.

Next, I would like to thank the members of my reading committee, Stan van Hoesel, An Caris, Aida Abiad Monge and Kenneth Sörensen, for their time and careful reading of this thesis. 
Furthermore, I would like to thank Karin van den Boorn, Vera Hoekstra and Yolanda Paulissen for helping me with all administrative problems. I further thank Brigitte Defesche for supporting me in the second half of my PhD. Our pleasant meetings always helped me to believe in myself again and brought me back on the right track. Moreover, a warm word of thanks to my fellow PhD students from the Department of Quantitative Economics at Maastricht University and from the Logistics Group at UHasselt for the great time we spent together. I was also lucky to always have great office mates. Christian, Hilde, Meng and Vincent, I really enjoyed to share an office with you, to exchange ideas, to have nice talks and to share some laughs. A special thanks to my paranymphs, Alex and Vincent. I am really happy that we became good friends. Vincent, I will miss the great time with spent together at University, especially in teaching periods. Alex, thank you for your support during my $\mathrm{PhD}$. Especially during the harder time of my $\mathrm{PhD}$ you were always there for me.

Moreover, I would like to thank my managers and colleagues from the CVG Team of Medtronic, where I worked the last two years while still writing on my thesis. I really appreciate your support by giving me the freedom to take the time for my thesis when needed and by backing me up. Special thanks also to Lisa for helping me with the Dutch summary.

I would also like to thank my friends outside the university. The great and fun time we spent together always helped me to forget about work, to relax and to enjoy life.

Finally, I would like to thank my closest and most important group of people, my family and my boyfriend Tim. You always believed in me, even in times I did not believe in myself. I would like to thank my parents for always encouraging me in everything I do, for supporting me to keep on going in the face of failure and for sharing the great moments of my PhD. Tim, thank you for always being there for me and for always listing to me (even if you just wanted to rest and to not think about work anymore). Your love and presence gave me the strength to achieve all this. 


\section{Contents}

1 Introduction 1

1.1 Supply Chain Collaboration . . . . . . . . . . . . . . . . 1

1.2 Supply Chain Collaborations: Successes and Failures . . . . . . . . 4

1.3 Research Objectives and Thesis Outline ........... 5

2 A Comprehensive Framework for Holistic Evaluations of Potential Supply Chain Collaborations 11

2.1 Introduction . . . . . . . . . . . . . . . . . . . . 11

2.2 Theoretical Development. . . . . . . . . . . . . . . . . . . 12

2.3 Research Design of the Case Studies . . . . . . . . . . . . 16

2.4 Discussion of Results . . . . . . . . . . . . . . . . . . 18

2.4 Benefits . . . . . . . . . . . . . . . . 19

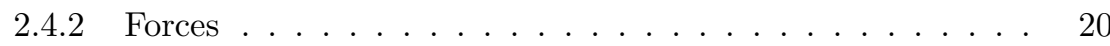

2.4.3 Enablers and Barriers . . . . . . . . . . . . . . . . 21

2.4 Risks . . . . . . . . . . . . . . . 26

2.4 .5 Further Findings . . . . . . . . . . . . . . . . 27

2.5 Conclusion, Implications and Future Research . . . . . . . . . 27

2.5.1 Theoretical Implications . . . . . . . . . . . . . . . 28

2.5 .2 Practical Implications . . . . . . . . . . . . . . . 28

2.5.3 Further Research Directions . . . . . . . . . . . . . . . . 29

2A References - Observed Industries, Perspectives and SCC Types . . 30

2B Case Study: Enablers/Barriers . . . . . . . . . . . . . . . 32

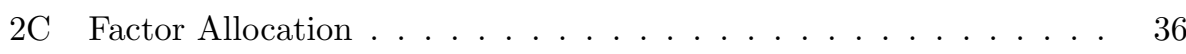

2C.1 Allocation Benefits . . . . . . . . . . . . . . 36 
2C.2 Allocation Forces . . . . . . . . . . . . . . . 41

2C.3 Allocation Enablers/Barriers ............. 44

2C.4 Allocation Risks . . . . . . . . . . . . . . . . . 64

2D Extract of the Interview Guide . . . . . . . . . . . . . 69

3 Disagreement on the Gain Sharing Method in Supply Chain Col$\begin{array}{ll}\text { laborations } & 71\end{array}$

3.1 Introduction . . . . . . . . . . . . . . . . . 71

3.2 Literature Review . . . . . . . . . . . . . . . . . . 73

3.2.1 Gain Sharing Methods . . . . . . . . . . . . . 73

3.2.2 Behavioral Decision-Making . . . . . . . . . . . 75

3.3 Research Procedure . . . . . . . . . . . . . . 76

3.3.1 Variable Selection . . . . . . . . . . . . . 78

3.3.2 Data Collection . . . . . . . . . . . . . . 81

3.3.3 Population and Sample Size Selection . . . . . . . . . . 82

3.3 .4 Data Analysis . . . . . . . . . . . . . 83

3.4 Statistical Analysis and Results . . . . . . . . . . . . . 86

3.4.1 Multicollinearity ............... 86

3.4.2 Regression . . . . . . . . . . . . . 88

3.4.3 Regression Manufacturer . . . . . . . . . . . 89

3.4.4 Regression LSP . . . . . . . . . . . . . . . . . 91

3.4.5 Regression Retailer . . . . . . . . . . . . . . . . 92

3.5 Result Discussion . . . . . . . . . . . . . . . . . 93

3.6 Conclusion, Implications and Future Research . . . . . . . . . . . . 95

3.6.1 Theoretical Implications . . . . . . . . . . . . . . . . . . . . 95

3.6.2 Practical Implications . . . . . . . . . . . . . . . 96

3.6.3 Further Research Directions . . . . . . . . . . . . 96

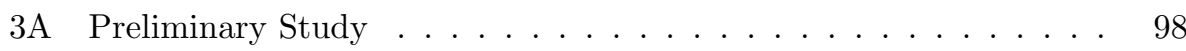

3B Online Survey ........................ 99

4 A Comprehensive Gain Sharing System Maximizing Satisfaction in Supply Chain Collaborations $\quad 105$

4.1 Introduction . . . . . . . . . . . . . . . . 105

4.2 Gain Sharing System . . . . . . . . . . . . 106

4.2 .1 Input . . . . . . . . . . . . . . 107

4.2.2 Gain Sharing Algorithm . . . . . . . . . . . . . 109

4.2 .3 Output ....................... 110 
4.3 Implementation . . . . . . . . . . . . . . . . . . . . . 110

4.3.1 Satisfaction Functions and Financial Information . . . . . . 110

4.3.2 Output Discussion . . . . . . . . . . . . . . . . . 113

4.4 System Stability . . . . . . . . . . . . . . . . . . . . 115

4.4.1 Fairness Properties . . . . . . . . . . . . 116

4.4.2 Sensitivity Analysis . . . . . . . . . . . . 117

4.5 Conclusion, Implications and Future Research . . . . . . . . . . . . 121

4.5.1 Theoretical Implications . . . . . . . . . . . . . . . . . . . . 121

4.5.2 Practical Implications . . . . . . . . . . . . . . . . . . 121

4.5.3 Further Research Directions . . . . . . . . . . . . . . 122

5 Urban Area Tessellations: Are Regular Tessellations Good for a Non-uniformly Distributed Demand? 123

5.1 Introduction . . . . . . . . . . . . . . . . . . . . . 123

5.2 Tessellations . . . . . . . . . . . . . . . . . 126

5.3 Methodology ... . . . . . . . . . . . . 128

5.3.1 City Layouts and Demand Distribution Models . . . . . . . 128

5.3.2 Suitable Tessellations . . . . . . . . . . . . . . 131

5.3.3 Performance of Tessellations on Different Layouts . . . . . . 133

5.4 Tessellation Comparison through Computational Experiments . . . 134

5.4.1 Triangular City Layout . . . . . . . . . . . . . . . . . . 134

5.5 Conclusion, Implications and Future Research . . . . . . . . . . . . 140

5.5.1 Theoretical Implications . . . . . . . . . . . . . . . . . . 141

5.5 .2 Practical Implications . . . . . . . . . . . . . . . . 141

5.5.3 Further Research Directions . . . . . . . . . . . . . . . 142

6 Final Conclusions and Further Research Directions 143

6.1 Final Conclusions . . . . . . . . . . . . . . . . 143

6.2 Further Research Directions . . . . . . . . . . . . . . . . 147

$\begin{array}{ll}\text { Bibliography } & 151\end{array}$

$\begin{array}{ll}\text { Valorization } & 165\end{array}$

$\begin{array}{lr}\text { Nederlandse Samenvatting } & 169\end{array}$

$\begin{array}{ll}\text { Curriculum Vitae } & 173\end{array}$ 



\section{Chapter 1}

\section{Introduction}

\subsection{Supply Chain Collaboration}

In the course of the last decades, companies started to realize the benefits of setting up a supply chain collaboration (SCC). Various challenges such as scarce resources, increased competition among the organizations and higher customer expectations forced companies to look outside their organizational boundaries to search for parties with whom they can collaborate (Cao and Zhang, 2011; Lambert et al., 1996). The need for launching an SCC is stressed by Whipple and Frankel (2000), who postulate that competition no longer takes place between firms but between supply chains. According to Horvath (2001), SCC is the driving force behind effective supply chain management (SCM). Cooper et al. (1997) defines SCM as the "integration of business processes from the end user through the original suppliers that provides products, services and information that add value for customers". In contrast, SCC can be defined as "two or more independent companies working jointly to plan and execute [...] operations with greater success than when acting in isolation" (Simatupang and Sridharan, 2002).

SCCs can be differentiated based on their structure and their level of integration. According to Simatupang and Sridharan (2002), three different SCC structures can be distinguished: vertical, horizontal and lateral. In a vertical SCC, two or more parties from different supply chain levels, such as the manufacturer, the logistics service provider (LSP) and the retailer, collaborate in order to improve the supply chain performance. Well-known examples of vertical SCCs are 
vendor managed inventory (VMI), efficient customer response (ECR) and collaborative planning, forecasting and replenishment (CPFR) (Simatupang and Sridharan, 2002). In contrast, horizontal SCCs occur when two or more unrelated or competing parties from the same supply chain level collaborate (Cruijssen et al., 2007b; Simatupang and Sridharan, 2002). According to Cruijssen et al. (2007b), examples of horizontal SCCs are manufacturers consolidation centers (MCCs) as well as joint route planning. Lateral SCCs aim at gaining more flexibility through combining and sharing capabilities of vertical and horizontal dimensions (Simatupang and Sridharan, 2002). An example of a lateral SCC is the synchronization of several manufacturers and LSPs in an effective transport network (Mason et al., 2007).

Beside a structural distinction, SCCs can be differentiated based on their level of integration. As shown in Figure 1.1, relationships between two or more parties range from arm's length relationships to full integration. In this thesis, relationships that can be identified as SCCs are situated in between arm's length relationships and full integration. This is in line with Cruijssen et al. (2007b) and Lambert et al. (1996).

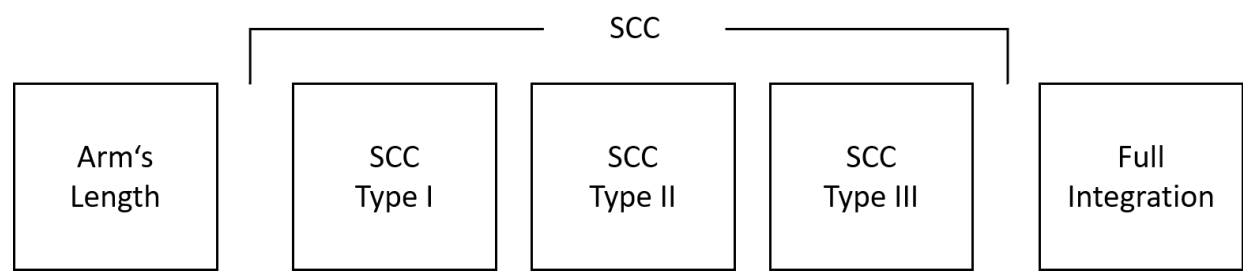

Figure 1.1: Relationship types (modified from Lambert et al. (1996))

An arm's length relationship is purely transactional and has no degree of collaboration since there is no incentive of joint commitment or operations (Kampstra et al., 2006; Lambert et al., 1996). An example of this kind of relationship is subcontracting. In the context of the transport and logistics industry, Cruijssen et al. (2007b) describe an arm's length relationship between two LSPs who subcontract capacity to each other. In contrast, full integration requires some sort of merging activity (Cruijssen et al., 2007b; Lambert et al., 1996). One example is PepsiCo, Incorporation (Inc.) which purchased restaurants from the brands Taco Bell, Corporation (Corp.), Pizza Hut, Inc. and Kentucky Fried Chicken (KFC) to ensure the distribution of its products in these restaurants (Lambert et al., 1996). In between these two extremes, three SCC types are situated differing in their level 
of integration (Lambert et al., 1996). In Table 1.1, key characteristics of these SCC types are outlined. SCC Type I refers to a short-term relationship between collaborative parties which perceive each other as coalition partners. Nevertheless, the collaboration is on a limited basis and only refers to the coordination of activities and planning. Furthermore, it involves just one division/function. In an SCC Type II, the activities and planning are not just coordinated but integrated. The collaboration is planned on a long-term horizon and involves multiple divisions/functions. In case a significant integration of the collaboration, it can be referred to as an SCC Type III. In this type of collaboration, the parties perceive each other as an extension of their own company. The collaboration has no fixed termination date and the collaborative parties share a significant level of operational integration.

Table 1.1: SCC types (based on the definitions outlined in Cruijssen et al. (2007b) and Lambert et al. (1996))

\begin{tabular}{|c|c|c|c|}
\hline & Level of Integration & Time Horizon & $\begin{array}{l}\text { Division/Function } \\
\text { Involvement }\end{array}$ \\
\hline $\begin{array}{l}\text { Type } \\
\text { I }\end{array}$ & $\begin{array}{l}\text { Solely coordination of } \\
\text { activities and planning }\end{array}$ & Short term & $\begin{array}{c}\text { Single } \\
\text { division/function }\end{array}$ \\
\hline $\begin{array}{c}\text { Type } \\
\text { II }\end{array}$ & $\begin{array}{l}\text { Integration of activities } \\
\text { and planning }\end{array}$ & $\begin{array}{l}\text { Long-term, but } \\
\text { finite length }\end{array}$ & $\begin{array}{c}\text { Multiple } \\
\text { divisions/functions }\end{array}$ \\
\hline $\begin{array}{l}\text { Type } \\
\text { III }\end{array}$ & $\begin{array}{l}\text { Integration on a } \\
\text { significant level }\end{array}$ & $\begin{array}{c}\text { No fixed } \\
\text { termination } \\
\text { date }\end{array}$ & $\begin{array}{l}\text { All organizational } \\
\text { divisions/functions }\end{array}$ \\
\hline
\end{tabular}

Within this thesis, strategic alliances are considered as another possible way to collaborate. An affiliation of a strategic alliance to one of the relationship types in Figure 1.1 is often discussed in literature. According to e.g. Nanda and Williamson (1995), strategic alliances relate to merged relationships and, hence, are assigned to the relationship level full integration. However, referring to Hagedoorn and Sadowski (1999), only $2.6 \%$ of all strategic alliances are merged relationships. According to this statement, a strategic alliance will be assigned to an SCC type; typically to Type III (Cruijssen et al., 2007b).

In this thesis, vertical, horizontal and lateral SCCs are considered. Chapter 2 deals with all three structural types. Chapters 3 and 4 focus on vertical SCCs, 
whereas Chapter 5 relates to horizontal SCCs. Regarding the level of integration, the focus is on the three SCC types, including strategic alliances, outlined in Table 1.1. The two extremes, arm's length relationships and full integration, are not considered to be SCCs and are therefore out of scope.

\subsection{Supply Chain Collaborations: Successes and Failures}

Several researchers (e.g. Boddy et al., 2000; Cao and Zhang, 2011; Lambert et al., 1996) outline sustainable competitive advantages that can be achieved through SCC. According to Lambert et al. (1996), SCC might leverage unique expertise and skills from all collaborative parties. Furthermore, a successful SCC can lead to lower costs in e.g. manufacturing, inventory and distribution (Horvath, 2001; Stank et al., 2001). Besides the reduction of costs, Stank et al. (2001) also mention an improved service performance as an SCC outcome. Other competitive advantages might be an increased customer satisfaction as well as a cycle time reduction (Daugherty et al., 2006). One example of a successful SCC in practice is the collaboration of Walmart Inc. and The Procter \& Gamble Company (P\&G), who experienced significant success through a joint forecast of sales for P\&G products at Walmart Inc. stores and a joint planning of corresponding replenishment strategies (Attaran and Attaran, 2007).

Despite the fact that SCC may have significant benefits, multiple collaborative incentives fail (Bititci et al., 2007). According to Sabath and Fontanella (2002), the "supply chain collaboration is at the same time the most used, the most frequently misunderstood, the most popular - and the most disappointing - strategy that has come along to date". In the context of horizontal SCCs, Schmoltzi and Wallenburg (2011) outline that 50 to $70 \%$ of all collaborations break down. Regarding SCCs in general, 50 to even $77 \%$ of all collaborations fail (Zineldin et al., 2015). In the literature, several reasons for unsuccessful SCCs are discussed. According to Fawcett et al. (2012), one main challenge for SCCs is a lack of trust. Sherman (1992) points out that one-third of all alliances fail due to a lack of trust among the collaborative parties. Another challenge mentioned in the scientific literature might be the power imbalance between SCC partners. In Bretherton and Carswell (2002), the issue of asymmetrical relationships is described. In the 
case of an asymmetrical relationship, the parties have different levels of power. As a result of this power imbalance, Bretherton and Carswell (2002) claim that these collaborations are less stable compared to symmetrical relationships. Furthermore, Autry (2011) highlights another SCC challenge: the identification and understanding of all relevant drivers and resistors before launching the SCC. In order to eliminate obstacles before they lead to a breakdown of the SCC, it is necessary to be aware of these impediments. In addition, according to Cruijssen et al. (2007a) and Leng and Parlar (2009), another main difficulty for the implementation and success of SCCs is a fair allocation of the coalition gain. If one party is not satisfied with or does not accept the assigned gain share, future collaborations are less likely to occur (Jap, 2001). Identifying the right party to collaborate with represents another major challenge for the implementation of a successful SCC (Cruijssen et al., 2007a). According to Barratt (2004) and Lambert et al. (1996), parties are not able to collaborate with too many potential parties due to the resource intensive nature of SCCs. Moreover, Brouthers et al. (1995) state that collaborating with an unsuitable partner might lead to more organizational damage than not collaborating. In order to select a suitable partner, parties need specific information about their potential coalition partners, such as organizational capabilities. Since this kind of information is often kept private, the partner selection process might be an expensive and difficult task (Cruijssen et al., 2007b, Verdonck, 2017). One practical example of an SCC which ended unsuccessfully is the collaboration between KLM Royal Dutch Airlines N.V. and Northwest Airlines (Bititci et al., 2007). Their dyadic relationship was not continued due to ineffective communication. General Motors Company and Daewoo also terminated their collaboration prematurely. A lack of trust in the relationship as well as a lack of understanding for each other could be identified as the main reasons for the terminated cooperation (Bititci et al., 2007).

\subsection{Research Objectives and Thesis Outline}

SCC has a huge potential. However, realizing its potential requires further investigation (Goffin et al., 2006). Moreover, the costs of SCC failures assert the need for an investigation on why successful SCCs are rare (Fawcett et al., 2015). In this context, it is essential to examine challenges for successful collaborations and to identify ways to deal with these challenges. As Baumeister et al. (2001) point out: adequate management of these SCC challenges has a greater impact on their 
success than focusing only on the development of successful SCCs. Understanding the various facets of challenges is crucial to maintain long-term SCC success (Samaha et al., 2011). In this thesis, three collaboration challenges are critically investigated and approaches to tackle these challenges are outlined: identification and understanding of drivers and resistors for launching an SCC, gain sharing and partner selection. The thesis contributes to existing literature in the following way. The focus of current SCC studies is on the positive aspects of collaborations rather than on SCC challenges (Cruijssen et al., 2007b; Lambert et al., 1999; Zineldin and Bredenlöw, 2003). Zineldin and Bredenlöw (2003) further mention a need to deeper investigate the problems and risks of coalitions to understand why so many SCCs break down. The literature on collaboration challenges often discusses the reasons for unsuccessful SCCs or evaluates the impact of challenges on the coalition performance (e.g. Fang et al., 2011; Richey Jr et al., 2010), but does not identify approaches to tackle the SCC challenges. Nevertheless, referring to Richey Jr et al. (2010), apart from understanding the challenges associated with the implementation and success of collaborations, it is important to understand how to overcome them.

As indicated in Section 1.2, one challenge for a successful SCC is the identification and understanding of all relevant drivers and resistors for launching a coalition. In order to eliminate a barrier before it leads to the breakdown of the collaboration, it is necessary to be aware of this obstacle before launching the SCC. Until now, a significant amount of literature discusses drivers and resistors for launching an SCC, in some cases, also for specific SCC structures, perspectives and/or industries. However, a comprehensive and structured study including all drivers and resistors for any potential SCC structure, perspective and industry is missing. Moreover, existing research is often ambiguous. For instance, it is ambiguous that the same term is used for various driver or resistor categories rather than having an unique term for each driver or resistor category. Secondly, some drivers are allocated to more than one driver category. Another shortcoming of the existing literature is incompleteness; drivers or resistors mentioned in one paper for a specific driver or resistor category are not mentioned in other studies for the same category. In response to these research gaps, Chapter 2 develops a comprehensive, structured and consistent framework of all potential drivers and resistors for launching an SCC for a broad range of SCC structures, industries and perspectives. The framework provides companies with the opportunity to 
more holistically evaluate the considered SCC and to identify collaborations with a high failure potential. In addition, the completeness of the framework is validated in the Dutch fast moving consumer goods (FMCG) industry. The literature on drivers and resistors for the FMCG industry is limited and up to now not all potential drivers and resistors have been investigated. The existing literature mainly focuses on barriers (Jharkharia and Shankar, 2005) and forces (de Leeuw and Fransoo, 2009). As for the investigation of the framework, all potential drivers and resistors for the Dutch FMCG industry are identified and examined, which in turn adds to the completeness of the developed framework.

Another major challenge for the implementation and success of SCCs is the fair allocation of coalition gains (Cruijssen et al., 2007b; Leng and Parlar, 2009). For a sustainable SCC, it is necessary that all collaborative parties are satisfied with their allocated shares and for them to feel that they receive a fair share of the coalition gain. Based on its importance, in this thesis the challenge of allocating the gain in a fair manner is discussed in Chapters 3 and 4 .

Several researchers already developed gain sharing methods in order to allocate the gain in such a way that everybody is satisfied with and accepts the assigned gain share. However, the existing literature does not investigate the actual acceptance levels of these gain sharing methods in practice. In response to this research gap, in Chapter 3 the acceptance levels of selected gain sharing methods in vertical three-echelon SCCs in the Dutch FMCG industry are investigated. Furthermore, the influence of behavioral decision-making aspects on the acceptance levels of these gain sharing methods is observed in order to explain the cause for the acceptance or rejection of the gain sharing method. For a long time, the predominant assumption in economics was that human beings think rational, which implies that decisions are made in a rational and consistent way (Sterman, 1989). However, due to limitations of available information, cognitive capabilities and time, human beings are endowed with a bounded rationality (Simon, 1979). Human beings tend to rely on heuristics or cognitive biases to handle complex problems (Schenk, 2011). The incorporation of behavioral research literature into SCM literature in Chapter 3 is an important research contribution. Until now, limited research dealing with the influence of human behavior, judgment and decision-making has been published in the fields of logistics and SCM (Tokar, 2010). However, to ensure practical validity, the integration of behavioral decision-making in SCM studies is necessary (Sterman, 1989; Tokar, 2010). 
Chapter 4 builds upon the research findings of Chapter 3. The survey in Chapter 3 reveals that no gain sharing method is accepted by and satisfies all collaborative parties. Furthermore, additional interviews with parties from the Dutch FMCG industry show that simple methods are preferred in practice. These findings are confirmed by the existing research, which outlines that game-theoretic gain sharing methods are perceived as too hard to understand and too complex to implement (Leng and Parlar, 2005). Although the need for simple gain sharing methods and the importance of the parties' satisfaction with the assigned gain share has been acknowledged both in theory (e.g. Verdonck et al., 2016) and practice, to the best of the author's knowledge existing gain sharing methods do not entirely resolve these two aspects. Therefore, in Chapter 4, a comprehensive, yet simple gain sharing system which focuses on the maximization of the parties' satisfaction using a minimax regret approach is developed.

The third SCC challenge which is investigated in this thesis is finding a suitable coalition partner (Cruijssen et al., 2007b). According to Brouthers et al. (1995), collaborating with an unsuitable party leads to more organizational damage than not collaborating. Furthermore, for companies active in the transport industry a broad geographical coverage is very important, but cannot always be achieved on an individual basis (Schmoltzi and Wallenburg, 2011). Bleeke and Ernst (1995) and Cruijssen et al. (2007a) indicate that the geographical coverage can be expanded through horizontal SCC. As such, one important aspect that should be taken into account in the partner selection process is the geographical dimension of potential cooperation partners. In this context, Bleeke and Ernst (1995) point out that a collaboration between companies that do not geographically share a customer market is more beneficial due to less competitive pressure. In line with this geographical perspective on partner selection, in Chapter 5 an approach for facility location problems is proposed which can be applied in the context of horizontal SCCs. This approach is briefly explained in the following.

In geometry, tessellations are used to cover a plane without gaps and overlaps using different shapes. Whenever a tessellation is made up of congruent regular polygons, researchers talk about regular tessellation (Grunbaum and Shephard, 1977). In the context of regular tessellation, whenever the demand for a commodity is evenly distributed, the preferred polygons are hexagons since they have the property to cover the plane without gaps and overlaps with the smallest number of polygons (Mallozzi et al., 2017; Schultz, 1970). However, in the course of the 
last decades, the population shifted from rural to urban areas, a trend know as urbanization (Cohen, 2006). As the regularity of the population has changed, the assumption of a uniformly distributed demand/population is not valid anymore. Therefore, in Chapter 5 the performance of regular tessellations as well as semiand demi-regular tessellations in the context of facility location problems is investigated under well-known non-uniform demand distributions, which are common in practice. In addition, based on the results of the performance analysis of the regular tessellations, two new expanding hexagonal tessellations are proposed for one common demand distribution. It is identified that these expanding hexagonal tessellations lead to a fair and cost-efficient way to locate facilities for a specific customer spread assuming a non-uniformly distributed demand.

The investigated tessellation as well as the new proposed expanding hexagonal tessellation approach support supply chain parties in the partner selection process by suggesting where the partner's existing or planned facilities should be preferably located in order to achieve a significant geographical coverage. Furthermore, since the introduced tessellations cover a plane without overlaps, it is ensured that the collaborative parties do not have overlaps in their customer bases, which will reduce the competitive pressure within the SCC (Bleeke and Ernst, 1995). The approach additionally reduces transport costs for each collaborative party, which in turn improves the SCC efficiency (Cruijssen, 2006).

Finally, Chapter 6 outlines general conclusions and further research directions. 



\section{Chapter 2}

\section{A Comprehensive}

\section{Framework for Holistic}

\section{Evaluations of Potential}

\section{Supply Chain}

\section{Collaborations $^{1}$}

\subsection{Introduction}

Referring to Autry (2011), an important SCC challenge is the identification and understanding of all potential drivers and resistors for launching a collaboration. In order to eliminate a certain barrier before it leads to the breakdown of the coalition, it is necessary to be aware of this obstacle. A significant amount of literature already investigated potential drivers and resistors for launching an SCC, in some cases for a specific industry, SCC structure and/or perspective. Ahmad and Ullah (2013) provide a literature review on potential SCC benefits and factors which enable a company to launch a collaboration. Next to that, Cruijssen et al. (2007a) discuss potential opportunities and impediments of horizontal coalitions from the LSP perspective, whereas de Leeuw and Fransoo (2009) describe external

\footnotetext{
${ }^{1}$ This chapter is based on the papers Jung et al. (2017) and Jung et al. (2018b)
} 
factors which force a supply chain party to launch a collaboration for selected industries and perspectives. Although prior research has widely discussed potential drivers and resistors to launch SCCs, a comprehensive study including all drivers and resistors for any potential SCC structure, industry and perspective is missing. Furthermore, most current work is prone to ambiguity and incompleteness, as outlined in detail in Section 2.2. In response to these research gaps, a comprehensive, structured and consistent framework listing all potential drivers and resistors for launching a collaboration is developed in this chapter using an extensive literature review. The framework provides companies with the opportunity to more holistically evaluate the considered collaboration and to identify collaborations with a high failure potential. In addition, the completeness of the framework is validated through case studies in the Dutch FMCG industry. In these case studies, companies within the Dutch FMCG industry are asked to identify all relevant drivers and resistors for SCCs they participated in. Literature examining potential factors for launching SCCs in the FMCG industry is scarce and until now not all drivers and resistors have been investigated. The existing literature mainly focuses on barriers (Jharkharia and Shankar, 2005) and forces (de Leeuw and Fransoo, 2009). For the validation of the framework, all relevant drivers and resistors for launching collaborations in the Dutch FMCG industry are identified. Providing this overview of drivers and resistors for the FMCG industry further adds to the completeness of the framework.

The remainder of this chapter is structured as follows. In Section 2.2, an extensive literature review is presented. The existing literature is critically discussed and a comprehensive, structured and consistent framework of drivers and resistors for launching SCCs is created. Moreover, to test the completeness of the framework, relevant drivers and resistors for the FMCG industry are investigated using a qualitative case study approach. The research methodology is explained in Section 2.3, followed by the analysis and discussion of the case study in Section 2.4. The chapter concludes with an outline of theoretical and practical contributions and directions for further research in Section 2.5.

\subsection{Theoretical Development}

Until now, a significant amount of literature identified relevant drivers and resistors for launching SCCs (e.g. Akintoye et al., 2000; Fawcett et al., 2008a). Neverthe- 
less, in none of these articles a comprehensive overview of all potential drivers and resistors for launching collaborations is presented. Furthermore, the existing literature is prone to ambiguity and incompleteness. In the literature, there are two kinds of ambiguity for the drivers. Firstly, the same terms are used for different driver categories and there are no unique terms for the identified driver categories. Examples are the terms drivers and driving forces. Ahmad and Ullah (2013) use the term drivers to define two driver categories. First, they use the term to define factors which enable someone to collaborate, like trust or commitment. Second, they use the term for expected benefits of successful collaborations such as improved customer service or increase in market share. Next to the term drivers, the authors also use the term driving forces for the expected benefits. However, the term driving forces is used by Fawcett et al. (2008b) to define factors which force a party to collaborate, like more demanding customers or economic globalization. Secondly, drivers are assigned to more than one driver category. An example is the factor trust, which is identified by Akintoye et al. (2000) as a factor which enables someone to collaborate, and by Beach et al. (2005) as an SCC outcome. For the resistors, a unique term and definition is also missing. Even though unlike the drivers, most studies only name one resistor category. However, even for this single category multiple terms such as barriers (e.g. Akintoye et al., 2000) or impediments (e.g. Cruijssen et al., 2007a) are used. Moreover, an incompleteness can be observed: in earlier research identified driver or resistor is not present in more recent literature. An example is the factor openness. It is identified as a factor which enables someone to collaborate by Barratt (2004), but it is not mentioned by Ahmad and Ullah (2013) in their literature review for the same category.

Due to the ambiguity, incompleteness as well as the fact that no paper discusses all potential drivers and resistors, the understanding and identification of all relevant drivers and resistors for a specific SCC represents a challenge for the collaborative parties. Therefore, a complete overview of all potential drivers and resistors for launching a collaboration is needed to holistically evaluate potential coalitions.

Drawing on prior research, a conceptual framework consisting of two umbrella terms is developed in this section. The first umbrella term is called drivers and the second one resistors. The term drivers represents the various driver categories identified in the literature. It is split into three categories. The first category, 
benefits, represents the expected benefits of a successful SCC, such as the benefit cost reduction (e.g. Akintoye et al., 2000). The second category, forces, contains external factors which force a party to collaborate, such as the force economic globalization (e.g. Fawcett et al., 2008b). The last category, enablers, includes factors which enable someone to collaborate and in addition have an effect on the SCC success, such as the factor trust (e.g. Akintoye et al., 2000). The distinction between the three driver categories is made to highlight the differences between the drivers. Benefits and forces are both motivating factors to collaborate. Nevertheless, there is a big difference. Benefits represent an intrinsic motivation, which means that the company decides to collaborate out of their own motivation (Lambert et al., 1996). Consequently, the benefits usually have a positive influence on coalitions. In contrast, the forces represent an extrinsic motivation. Here, a party is forced to collaborate and therefore a change in management practice towards more collaboration is dictated but not necessarily wanted by the party itself. This might have a negative influence on collaborations (Fawcett et al., 2008b). A strong motivation to build an SCC is not enough. Hence, in addition to the motivating factors enablers are required. Enablers increase the probability of success and therefore have a positive influence on collaborations (Lambert et al., 1996; Richey Jr et al., 2010). The umbrella term resistors is used to represent all retarding factors for SCC. The resistors are divided into two categories, which both have a negative influence on collaborations. The first category, barriers, consists of impediments that can obstruct the SCC and are known before the collaboration launches. An example is the factor lack of commitment (e.g. Akintoye et al., 2000). Based on the extensive literature review, a connection between the driver category enablers and the resistor category barriers is observed. For each enabler, a corresponding barrier can be identified (Walker et al., 2008). An example is the enabler information sharing and the barrier lack of information sharing (e.g. Fawcett et al., 2008a). To show this connection, the factors of these two categories are combined. When evaluating coalitions, factors will be identified either as an enabler, presence of..., or as a barrier, lack of.... The second category is called risks. Risks are future-oriented and include events that might occur in the future, but are unknown yet, like the risk recession (e.g. Landeros et al., 1995). The distinction between barriers and risks is also mentioned by Evans (2012) in the context of strategic planning. Given that barriers are already known at the moment in time when parties decide whether to collaborate or not, each party can already take actions against the barriers prior to the collaboration. In contrast, 
the risks are uncertain at this point in time. As a result, the decision to collaborate or not is dependent on the decision makers and their risk preferences (risk averse, risk neutral or risk loving).

Based on a review of 36 studies from 1990 to 2015, see Appendix 2A, 65 benefits, 25 forces, 100 enablers, 81 barriers and 64 risks were identified. After an extensive literature review based on key words such as barriers or drivers, the papers were selected based on the impact factor. Papers published in journals with an impact factor of 2 or higher were taken into consideration. To create a clear overview of all potential drivers and resistors, the factors were assigned to so-called general factors. Reported benefits like increased customer satisfaction, enhanced delivery performance and increased customer responsiveness (Fawcett et al., 2008a) all refer to improvements with regard to the customers and were assigned to the general factor customer improvements. The 335 identified drivers and resistors were assigned to 73 general factors; 10 general factors for the benefits, 11 for the forces, 35 for the enablers/barriers and 17 for the risks. Figure 2.1 shows the resulting conceptual framework including definitions and explanations of the influences of the categories on the SCC as well as all identified general factors for each category. Following the structure of the framework, in Appendix $2 \mathrm{C}$ each factor identified in the literature review as well as in the qualitative case study, which is introduced in Section 2.3, is assigned to the general factors of each category. Moreover, a clear definition for each general factor is provided. 


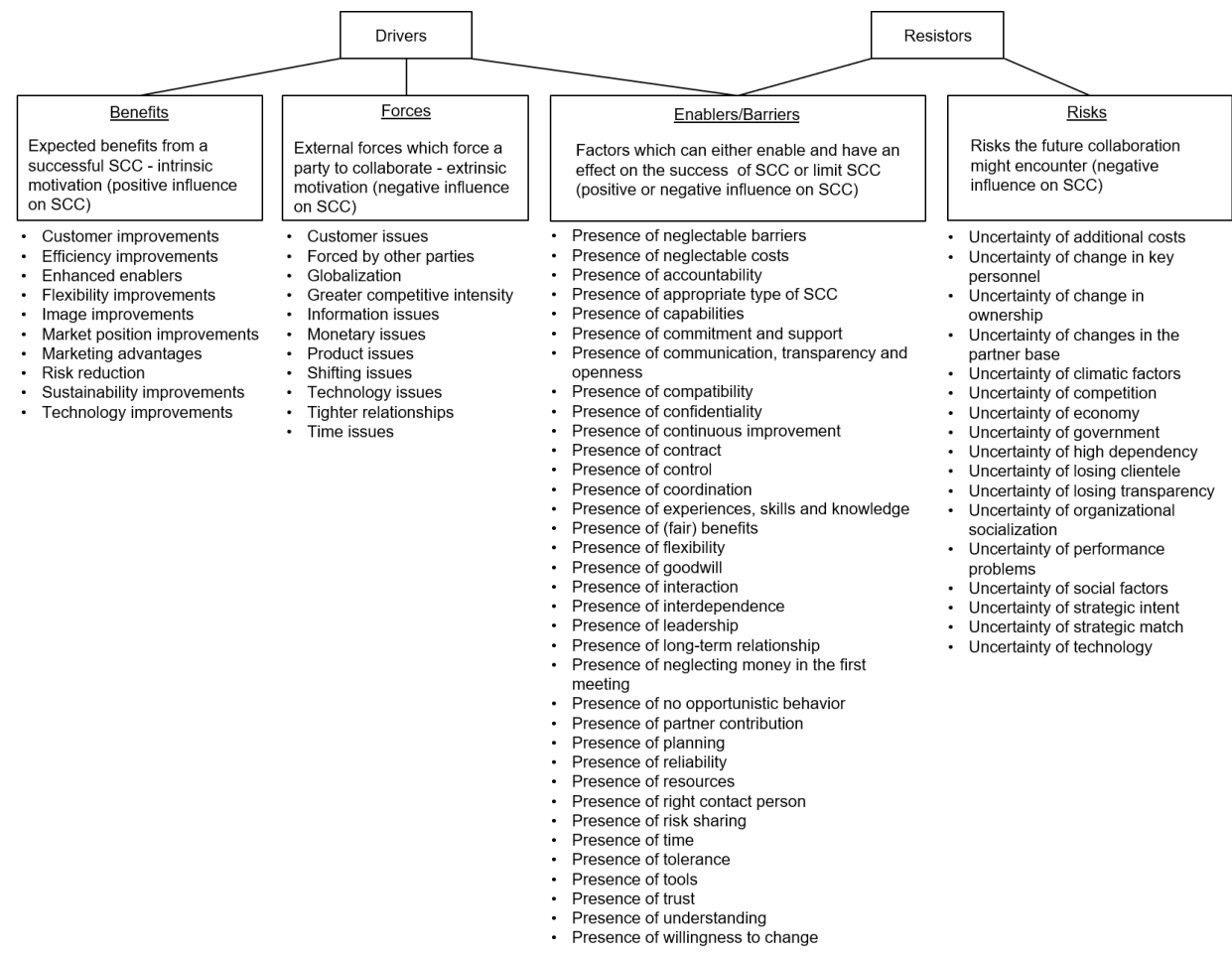

Figure 2.1: Complete framework

The drivers and resistors may differ for each party in a collaboration as well as in each industry and for each SCC type (Lambert et al., 1999). Appendix $2 \mathrm{~A}$ provides an overview of the investigated perspectives, industries as well as SCC types of the selected papers. In addition, if differences between perspectives, industries and/or SCC types were made for the drivers and resistors factors within the paper, the considered perspective, industry and/or SCC type is specified in the last column of the tables in Appendix 2C.

\subsection{Research Design of the Case Studies}

The completeness of the conceptual framework is validated by investigating drivers and resistors to launch collaborations in the Dutch FMCG industry. Coalitions are very important for this industry. Referring to de Kok et al. (2015): "[t]he FMCG sector is core to the wealth and well-being of the developed countries". Nearly $20 \%$ of the total ton kilometers transported in the European Union (EU) 
are goods from the FMCG industry. Without collaborating with supply chain partners, companies in the FMCG industry are not able to be competitive on the dynamic market. As a result, successful collaborations are essential for this industry (de Kok et al., 2015). Since the literature about relevant drivers and resistors for the FMCG industry is scarce and in addition not all driver and resistor categories have been investigated, this research is exploratory and necessitates a qualitative method (Eisenhardt, 1989); more precisely, a qualitative case study research approach is used. Qualitative case study research provides researchers with a tool to investigate a complex phenomenon within its real-life context (Yin, 2013). For the data collection, individual semi-structured in-depth interviews are used, which is a common way to explore practice and to collect data $(\mathrm{Qu}$ and Dumay, 2011; Yin, 2013).

Interviews were held with representatives of companies from the Dutch FMCG industry that participated in a logistics competition for manufacturers, LSPs and retailers in the Netherlands. The goal of the competition was to reduce the truck cycle time at the retailer's distribution center through SCCs. Out of the 26 participating parties, 20 accepted the interview request. Among the participants are seven manufacturers, six LSPs and seven retailers. This allows the researchers to investigate different perspectives. The majority of the interviews were conducted with the supply chain or logistics managers of the companies. The interviews started with general questions about the company and SCCs in general and were followed by questions about drivers and resistors for launching a specific SCC. The participants were free to mention several SCCs in which they participated. Furthermore, drivers and resistors for failed SCCs could also be specified. In total, 71 SCCs were mentioned by the participants. Among them were seven unsuccessful collaborations. The focus of several studies is on investigating dyadic relationships, but the attention has shifted to networks as well (Steinfield et al., 2011). In this study, different collaborations are investigated, as well. Among them are networks, such as triangular relationships, where three independent but connected parties are collaborating with each other. The interviews were mainly conducted face-to-face by visiting the companies' headquarters, production locations or distribution centers in the Netherlands. Due to time constraints, three interviews were conducted via telephone.

In order to ensure cross-case comparability, an interview guide was developed 


\section{A Comprehensive Framework for Holistic Evaluations of Potential Supply Chain Collaborations}

to provide directions during the interviews. However, the interviewer was also free to adapt or add certain questions during the interviews and the interviewee had a great deal of flexibility in how to reply. An extract of the interview guide is provided in Appendix 2D. The interview guide was made available to the participants one week before the interview to give the managers sufficient time for preparation.

In order to reduce the possibility of biases and to increase the credibility of the research findings, strategies introduced by Johnson (1997) as well as by Noble and Smith (2015) were applied. Firstly, the strategy multiple investigators was used for the data collection. At least two researchers were present at every interview. Secondly, a strategy called participants feedback was applied. All interviews were recorded by audio-recording or video and in addition notes were taken during the interviews. Based on the gathered information, a report of each interview was prepared and sent back to the interviewees for verification and to confirm that the researchers' interpretations were consistent with the respondents' interpretations.

After a detailed case study write-up for each case, the data were analyzed using cross-case analysis. The key to a good cross-case comparison is looking at the data in many divergent ways. Eisenhardt (1989) discusses three cross-case tactics. One tactic is to select categories and then look for within-group similarities coupled with intergroup differences. Dimensions can be suggested by the literature or can simply be chosen by the researcher. A second tactic is to group the cases and then list the similarities and differences between them. Finally, data can be divided by data source. The first two cross-case tactics were used, as this enabled an analysis of the elements as identified in the literature in addition to identifying the new elements from practice. This way, the probability of capturing the findings, which may exist in the data, is enhanced. After categorizing the factors mentioned in the interviews and comparing them with the general factors of the conceptual framework, the factors were analyzed by category (benefits, forces, enablers/barriers and risks) and party (manufacturers, LSPs and retailers).

\subsection{Discussion of Results}

In this section, the cross-case analysis is presented. The factors reported in the interviews are included in the framework and assigned to the right general factor. If the appropriate general factor was not defined before, this general factor is added 
to the framework. Results are discussed by category.

\subsubsection{Benefits}

Table 2.1 shows all benefits stated by the interviewees. The mentioned benefits are classified per party - LSP, manufacturer (MA) and retailer (RE) - and whether the factor was mentioned by a party who actually launched the SCC (C) or not (NC). The intrinsic motivation for launching collaborations in the FMCG industry does not significantly differ from the intrinsic motivation in other industries. All mentioned benefits are identified as, sometimes new, examples of the general factors in the conceptual framework (see Appendix 2C.1).

In the Dutch FMCG industry, the main motivation to launch a collaboration is cost reduction. This benefit has been mentioned as an important factor for parties to launch a collaboration in the literature as well. Cruijssen et al. (2007a) identify cost reduction as an important motivation factor for parties to launch an SCC in the logistics industry. The same holds for the construction (Akintoye et al., 2000) and the chemical industry (Reniers et al., 2010). Cost reduction, which is assigned to the general factor efficiency improvements, was mentioned as an intrinsic motivation factor for 18 SCCs. Half of it was stated by LSPs. Cruijssen (2006) points out that LSPs are concerned about their high transport costs. Therefore, it is reasonable that LSPs collaborate in order to reduce their costs. Moreover, by looking at the remaining benefits mentioned by the LSPs, it is obvious that this party is mainly driven by efficiency improvement factors; sustainability improvement seems to be less relevant for the LSPs. This is in line with the literature review by Cruijssen et al. (2007b), where the LSP perspective is observed in the transport and logistics sector. The majority of the outlined benefits are factors belonging to the general factor efficiency improvements.

The second most frequently mentioned general factor in the case studies is sustainability improvements. For some parties, sustainability is even more important than cost reduction (interview with one manufacturer on 28.05.2015). Due to an increased consciousness of the climate change, parties are imposing pressure on their suppliers to manage their greenhouse gas emissions as one of the conditions for doing business with them (Shaw et al., 2012). For the general factor sustainability improvements, the dominant factor is $\mathrm{CO}_{2}$ reduction. According to de Kok et al. (2015), in the FMCG industry retailers demand high-frequency shipments to their distribution centers and a low truck utilization efficiency. As a result, 


\section{A Comprehensive Framework for Holistic Evaluations of Potential Supply Chain Collaborations}

it is reasonable that sustainability improvement, especially $\mathrm{CO}_{2}$ reduction, is an often-named intrinsic motivation factor.

All benefits, except for three factors, the benefits were mentioned in the context of successful SCCs initiatives (see columns "C" and "NC"). This supports the finding that benefits have a positive influence on SCCs. Moreover, the interviews showed that some parties also launched collaborations without any intrinsic motivation. In the majority of these cases, forces instead of benefits were mentioned.

Table 2.1: Allocation of the benefits

\begin{tabular}{l|l|lll|ll}
\hline General Factors & Factors & LSP & MA & RE & C & NC \\
\hline \hline Customer & Increased customer & 1 & 1 & & 2 & \\
improvements & satisfaction & & & & & \\
\hline Efficiency & Cost reduction & 9 & 3 & 7 & 18 & 1 \\
improvements & Efficiency improvement & 3 & 1 & 4 & 8 & \\
& Employee reduction & & & 1 & 1 & \\
& Increased quality & & & 1 & 1 & \\
& Time reduction & 1 & & 5 & 4 & 2 \\
\hline Enhanced enablers & Increased transparency & 1 & & & 1 & \\
& Stronger partnership & 1 & & & 1 & \\
\hline Image improvements & Image improvement & & 2 & & 2 & \\
\hline Sustainability & CO 2 reduction & 2 & 2 & 3 & 7 & \\
improvements & Sustainability & 1 & 2 & & 3 & \\
\hline Technology & New possibilities/ & & & 1 & 1 & \\
improvements & technologies & & & & & \\
\hline
\end{tabular}

\subsubsection{Forces}

All extrinsic motivation factors identified by parties from the Dutch FMCG industry during the interviews (see Table 2.2) are assigned to already existing general factors (see Appendix 2C.2).

Unlike the intrinsic motivation, only two different extrinsic motivation factors were mentioned by the parties from the Dutch FMCG industry. Furthermore, exclusively manufacturers and LSPs stated extrinsic motivation factors to launch SCCs. The dominant force mentioned by these parties is that they were forced by the retailers to launch a specific collaboration. Three LSPs and five manufactur- 
ers were forced by retailers to, for example, use a specific software. One supply chain manager stated that they had "no choice", they were "forced by the retailer" (interview with one manufacturer on 14.04.2015). This might be due to the powerful position of the retailer in the Dutch FMCG industry, as outlined by another manufacturer: "the retailer is the most powerful party in the supply chain" (interview with one manufacturer on 24.04.2015). This is in line with the research by Adolfsson and Solarz (2005). They investigate relationships between suppliers and a retailer in the Swedish FMCG industry. Adolfsson and Solarz (2005) outline that the retailer has a powerful position in the Swedish FMCG industry and that suppliers are highly influenced by retailers. The retailer is highly intrinsically motivated. This finding contradicts the research by Walker et al. (2008). In their article, the authors identify important drivers and resistors for retailers to launch a green SCC. In this context, the retailers were not exclusively intrinsically motivated, but also often forced to launch the green collaboration. However, retailers in the FMCG industry seem to be mainly intrinsically motivated.

The analysis revealed that whenever a retailer forced an LSP and/or a manufacturer to join the collaboration, a collaboration launched although this was the only mentioned motivating factor for the parties to launch the SCC and apart from it only resistors were named. This stresses the powerful position of the retailer.

Table 2.2: Allocation of the forces

\begin{tabular}{l|l|cc|cc}
\hline General Factors & Factors & LSP MA RE & C NC \\
\hline \hline Forced by other parties & $\begin{array}{l}\text { Demanded by } \\
\text { manufacturer } \\
\text { Forced by retailer }\end{array}$ & 1 & & & 1 \\
& 3 & 5 & & 8 \\
\hline
\end{tabular}

\subsubsection{Enablers and Barriers}

In total, 48 enablers and barriers were mentioned during the interviews. For the other three categories, fewer factors were mentioned (12 benefits, 2 forces and 9 risks), which stresses the importance of the enabler/barrier category. Due to the amount of mentioned factors, in this section only the most frequently mentioned general factors are outlined. The total overview is presented in Appendix 2B. Unlike the other categories, not all identified factors for the category enablers/ barriers can be assigned to existing general factors. Twelve times, parties from the 


\section{A Comprehensive Framework for Holistic Evaluations of}

Potential Supply Chain Collaborations

Dutch FMCG industry claimed that talking about money in the first meeting leads to rough negotiations and, in some cases, even result in not launching the SCC. As one retailer mentioned: "if you are speaking about money at the beginning, the collaboration will immediately end" (interview with one retailer on 22.04.2015). For this factor, no academic evidence has been found. Many researchers already discussed the problem of a fair gain share allocation (e.g. Cruijssen et al., 2007a), but it has not been mentioned that this is especially a problem in the first meeting. Hence, an additional general factor was added, presence of neglecting money in the first meeting, if it is an enabler and lack of neglecting money in the first meeting, otherwise. All other identified factors provide new examples for already existing general factors (see Appendix 2C.3).

In the Dutch FMCG industry, especially organizational compatibility and trust are important enablers for launching SCCs. As one manager mentioned: "trust is most important" (interview with one retailer on 15.05.2015). Another interviewee also outlined that "without trust, it is not possible to collaborate" (interview with one LSP on 22.04.2015). In addition, it was stated that without organizational compatibility it is nearly impossible to collaborate (interview with one LSP on 13.05.2015). Other important enablers mentioned by the parties from the Dutch FMCG industry are transparency and interdependence. In the literature, it is often stated that in addition to these enablers, commitment is very important to launch collaborations (e.g. Mohr and Spekman, 1994). However, in the Dutch FMCG it seems to be less relevant, since it was only identified as an enabler for seven SCCs, see Appendix 2B.

Unlike the enablers, more variety exists for the barriers indicating that barriers are mostly individual and therefore highly dependent on the party and the specific situation. The major barrier for parties from the Dutch FMCG industry to launch an SCC is the lack of organizational compatibility. This is in line with former literature. The majority of studies discussing barriers to launch a collaboration also named lack of compatibility as a barrier (e.g Lambert et al., 1999; Simatupang and Sridharan, 2002). Furthermore, decreased efficiency, lack of using same technology and upfront investments are additional relevant factors. Another important barrier which was mentioned by parties from all echelons is the difficulty to establish a fair allocation of the gains. As outlined by some interviewees: "gain sharing is very difficult" (interview with one retailer on 22.04.2015) and "one of the bottle- 
necks was to speak about gain sharing" (interview with one LSP on 30.04.2015). Cruijssen et al. (2007a) referred to this barrier as one of the most severe barriers for the implementation and success of SCCs. In the context of fair gain sharing, interviewees additionally outlined the importance of open communication and transparency. According to one retailer, "basis for fair gain sharing is open communication" (interview with one retailer on 15.05.2015). This is stressed by another retailer and one LSP, who mentioned that transparency is an important prerequisite for fair gain sharing (interview with one retailer on 28.04.2015; interview with one LSP on 11.06.2015). The connection between transparency/open communication and fair gain sharing was also identified by Cruijssen (2006). The majority of the factors were either identified as an enabler for a specific SCC or as a barrier. However, a few parties also identified the same factor as enabler and barrier for one collaboration. In these cases, the parties outlined the general importance of an enabler to launch a collaboration. However, for the specific SCC the factor was mentioned as a barrier. The majority of these factors can be assigned to collaborations where the retailer forced parties to use specific software. In these cases, the manufacturers and LSPs outlined the general importance of organizational compatibility, but simultaneously mentioned the lack of organizational compatibility for the vertical collaboration.

By looking at the difference between collaboration and non-collaboration factors, it can be observed that the barrier upfront investments was often mentioned as a barrier for failed initiatives to SCC.

Table 2.3: Allocation of the enablers/barriers

\begin{tabular}{|c|c|c|c|c|c|c|c|}
\hline General Factors & $\begin{array}{l}\text { Enablers/ } \\
\text { Barriers }\end{array}$ & Factors & LSF & MA & $\mathrm{RE}$ & $\mathrm{C}$ & $\mathrm{NC}$ \\
\hline $\begin{array}{l}\text { Presence of } \\
\text { neglectable } \\
\text { costs }\end{array}$ & $\begin{array}{l}\text { Lack of } \\
\text { neglectable } \\
\text { costs }\end{array}$ & $\begin{array}{l}\text { High investments in } \\
\text { technology } \\
\text { Relationship-specific } \\
\text { upfront investments }\end{array}$ & 2 & 2 & 1 & 2 & $\begin{array}{l}1 \\
4\end{array}$ \\
\hline $\begin{array}{l}\text { Presence of } \\
\text { communication, } \\
\text { transpar- } \\
\text { ency and } \\
\text { openness }\end{array}$ & $\begin{array}{l}\text { Presence of } \\
\text { communica- } \\
\text { tion, trans- } \\
\text { parency and } \\
\text { openness }\end{array}$ & $\begin{array}{l}\text { Honesty } \\
\text { Information } \\
\text { sharing } \\
\text { Information } \\
\text { technology } \\
\text { Transparency }\end{array}$ & $\begin{array}{l}1 \\
1\end{array}$ & 2 & 2 & $\begin{array}{l}1 \\
5\end{array}$ & \\
\hline
\end{tabular}


2 A Comprehensive Framework for Holistic Evaluations of Potential Supply Chain Collaborations

Table 2.4: Allocation of the enablers/barriers

\begin{tabular}{|c|c|c|c|c|c|c|c|}
\hline $\begin{array}{l}\text { General } \\
\text { Factors }\end{array}$ & $\begin{array}{l}\text { Enablers/ } \\
\text { Barriers }\end{array}$ & Factors & LSP & MA & $\mathrm{RE}$ & $\mathrm{C}$ & $\mathrm{NC}$ \\
\hline $\begin{array}{l}\text { Presence of } \\
\text { communica- } \\
\text { tion, trans- } \\
\text { parency and } \\
\text { openness }\end{array}$ & $\begin{array}{l}\text { Lack of } \\
\text { communication, } \\
\text { transparency } \\
\text { and openness }\end{array}$ & $\begin{array}{l}\text { Difficulty to } \\
\text { share } \\
\text { confidential } \\
\text { information } \\
\text { Lack of } \\
\text { communication } \\
\text { Lack of } \\
\text { communication } \\
\text { between RE } \\
\text { and LSP in } \\
\text { triangular } \\
\text { relationships } \\
\text { Lack of } \\
\text { transparency } \\
\text { Lack of using } \\
\text { same } \\
\text { technology }\end{array}$ & 2 & 2 & 2 & $\begin{array}{l}1 \\
3\end{array}$ & 1 \\
\hline \multirow[t]{2}{*}{$\begin{array}{l}\text { Presence of } \\
\text { compatibility }\end{array}$} & $\begin{array}{l}\text { Presence of } \\
\text { compatibility }\end{array}$ & $\begin{array}{l}\text { Common goals } \\
\text { Organizational } \\
\text { compatibility } \\
\text { Shared values } \\
\text { Strategic fit }\end{array}$ & $\begin{array}{l}4 \\
5\end{array}$ & $\begin{array}{l}1 \\
7 \\
1 \\
1\end{array}$ & $\begin{array}{l}2 \\
5\end{array}$ & $\begin{array}{c}7 \\
17 \\
1 \\
1 \\
5\end{array}$ & \\
\hline & $\begin{array}{l}\text { Lack of } \\
\text { compatibility }\end{array}$ & $\begin{array}{l}\text { Difficulty to } \\
\text { work with } \\
\text { competitors } \\
\text { Lack of } \\
\text { common goals } \\
\text { Lack of } \\
\text { organizational } \\
\text { compatibility } \\
\text { Power } \\
\text { imbalance }\end{array}$ & 3 & 1 & $\begin{array}{l}3 \\
9\end{array}$ & 1 & 1 \\
\hline
\end{tabular}


Table 2.5: Allocation of the enablers/barriers

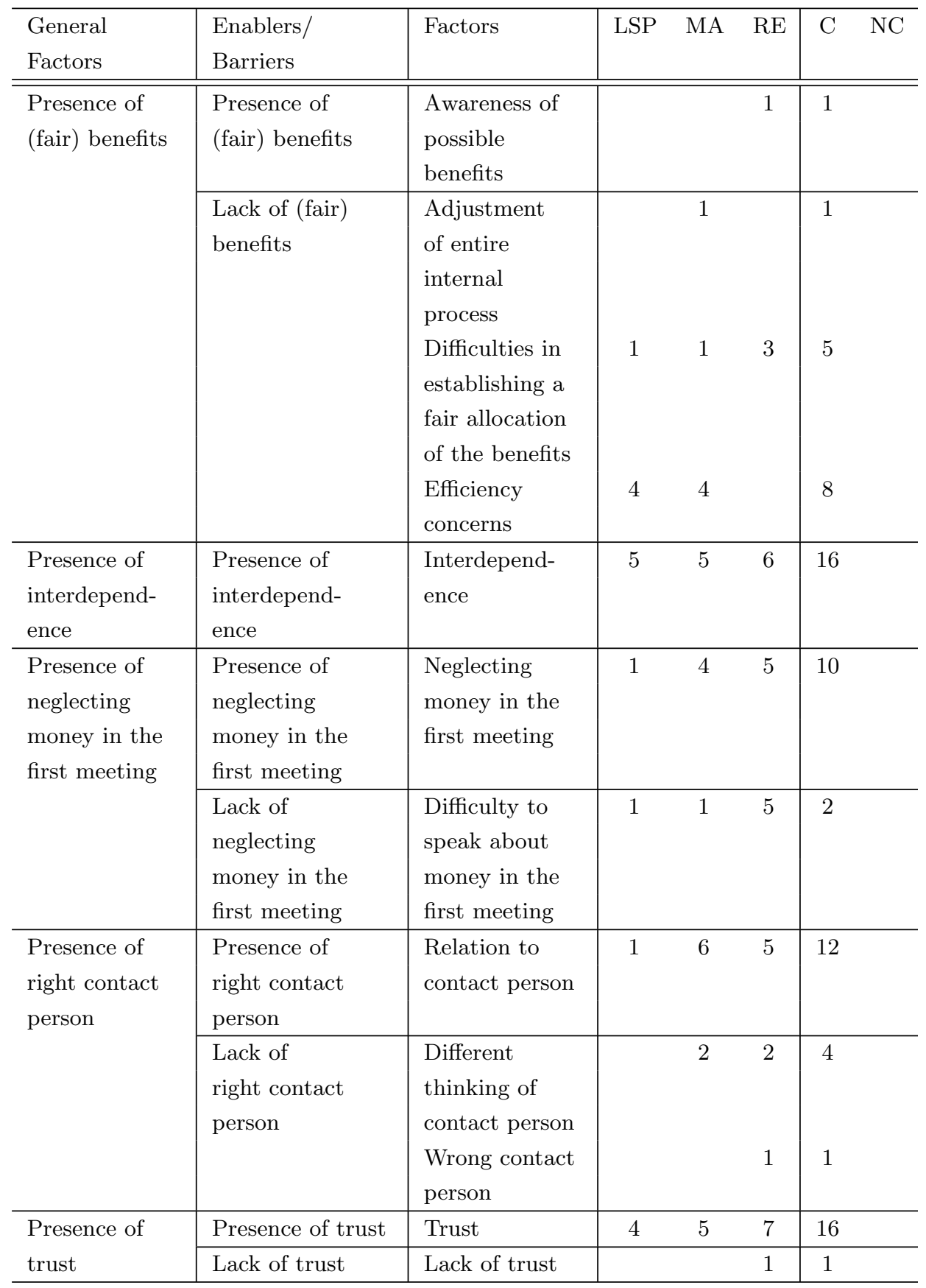




\section{A Comprehensive Framework for Holistic Evaluations of Potential Supply Chain Collaborations}

\subsubsection{Risks}

The identified risks (Table 2.6) can be assigned to already existing general factors (see Appendix 2C.4).

Only a few risks were mentioned by the interviewed parties. In the literature, also only a fairly limited number of studies discuss potential risks. The most frequently mentioned risk is dependency. The parties often stated that dependency combined with short-term contracts, which are common in this industry, results in high risks (interview with one LSP on 30.04.2015). Dependency has been mentioned as an important risk by Niederkofler (1991) as well.

Moreover, it has been identified that despite the risks the SCCs were launched in all cases, which might indicate that risks are not the main reason for rejecting an SCC.

Table 2.6: Allocation of the risks

\begin{tabular}{|c|c|c|c|c|c|}
\hline General Factors & Factors & LSP & MA & $\mathrm{RE}$ & $\mathrm{C} \quad \mathrm{NC}$ \\
\hline $\begin{array}{l}\text { Uncertainty of additional } \\
\text { costs }\end{array}$ & Risk of additional costs & 1 & 2 & 2 & 5 \\
\hline $\begin{array}{l}\text { Uncertainty of changes } \\
\text { of key personnel }\end{array}$ & $\begin{array}{l}\text { Risk of party leaving } \\
\text { SCC during } \\
\text { collaboration }\end{array}$ & & 1 & 2 & 3 \\
\hline $\begin{array}{l}\text { Uncertainty of high } \\
\text { dependency }\end{array}$ & $\begin{array}{l}\text { Contract uncertainty; } \\
\text { parties are mutually } \\
\text { dependent } \\
\text { Risk of dependency }\end{array}$ & 2 & 2 & 2 & $\begin{array}{l}4 \\
6\end{array}$ \\
\hline $\begin{array}{l}\text { Uncertainty of changes } \\
\text { in transparency }\end{array}$ & $\begin{array}{l}\text { Uncertainty of losing } \\
\text { transparency } \\
\text { Risk of confidential } \\
\text { information security }\end{array}$ & 1 & 1 & & $\begin{array}{l}1 \\
1\end{array}$ \\
\hline $\begin{array}{l}\text { Uncertainty of } \\
\text { performance problems }\end{array}$ & $\begin{array}{l}\text { Data reliability } \\
\text { Quality performance } \\
\text { problems } \\
\text { Uncertainty of outcome }\end{array}$ & & & $\begin{array}{l}2 \\
1 \\
1\end{array}$ & $\begin{array}{l}2 \\
1 \\
1\end{array}$ \\
\hline
\end{tabular}




\subsubsection{Further Findings}

To evaluate a potential SCC, it is important for parties to be aware of all potential factors. However, it is not necessarily the case that all (general) factors, except for the (general) factors of the category enablers/barriers, are relevant in every SCC; it depends on the party, its industry and the SCC structure. During the interviews, differences between the parties, but also between SCC structures were identified. An example for a special factor for one SCC structure is the following: Four LSPs intended to launch a horizontal SCC. As a barrier, legal barriers was mentioned. This is a specific barrier for a horizontal SCC. The problem is that the European Commission competition law prohibits any agreements between parties that are restrictive of competition like price agreements etc. (Cruijssen et al., 2007b). By law, competition is considered to be restricted if the collaborative parties together exceed a market share of 20\% (Commission, 2012). Moreover, it is possible that an entire category is not relevant for a specific party. The findings show that especially when parties are forced to collaborate, the intrinsic motivation to launch an SCC is often absent. Overall, it is important to recognize that each SCC and party is individual and therefore for every collaboration the relevant drivers and resistors have to be identified by each party individually.

\subsection{Conclusion, Implications and Future Research}

As outlined by one party during the interviews: "collaborations are crucial and necessary to be competitive on the dynamic market" (interview with one LSP on 15.04.2015). However, apart from advantages, SCCs bring along challenges. In this chapter, the challenge of identifying and understanding all important drivers and resistors for launching SCCs has been investigated. Overcoming this challenge is important in order to be able to eliminate e.g. a barrier before it leads to the failure of an SCC. For this purpose, an awareness of the barriers is essential. This awareness can be gained by means of a complete overview of all potential drivers and resistors. In this chapter, based on an extensive literature review a comprehensive, structured and consistent framework including all drivers and resistors for launching an SCC for all SCC structures, industries and perspectives mentioned in the literature has been developed. Furthermore, the completeness of the framework has been validated by investigating important drivers and resistors in the Dutch FMCG industry. It has been outlined that the drivers and resistors mentioned in the interviews are in line with the ones in the general framework ex- 
cept for one general factor of the category enablers/barriers. In the Dutch FMCG industry, supply chain actors perceive that it is difficult to talk about money in the first meeting. After adding a further general factor, which is called presence of neglecting money in the first meeting if it is an enabler and lack of neglecting money in the first meeting if it is a barrier, the framework is complete for the investigated industries, SCC types and perspectives. This complete framework provides the parties with the opportunity to more holistically evaluate the considered SCC and identify collaborations with high failure potential.

\subsubsection{Theoretical Implications}

The contribution of this chapter to the existing SCM literature is twofold. First, the chapter provides the first comprehensive framework of all potential drivers and resistors for launching SCCs mentioned in the literature. Although prior research widely discussed potential drivers and resistors, until now a study including all drivers and resistors for launching SCCs for all investigated SCC structures, industries and perspectives is missing. Moreover, the current literature displays a level of incompleteness; factors for a specific category mentioned in one paper are not mentioned in other articles. In addition, in this research ambiguities of two different kinds have been identified in the current literature. The first kind of ambiguity is that the same terms are used for various driver and resistor categories and that there are no unique terms for the driver and resistor categories. The second kind of ambiguity is that factors are assigned to more than one category. In this chapter, a structured framework with a consistent terminology and definitions has been developed, which enriches the SCM literature with an extensive specification of all potential drivers and resistors for launching SCCs. The completeness of the framework has been investigated in the Dutch FMCG industry. Until now, no research investigated all relevant drivers and resistors for the FMCG industry. Therefore, the second research contribution of this chapter is the investigation of all potential drivers and resistors for launching an SCC in the FMCG industry.

\subsubsection{Practical Implications}

The structured and complete framework supports parties to more holistically evaluate the considered SCC and identify collaborations with high failure potential. The evaluation of an SCC is enhanced through an improved understanding and identification of the drivers and resistors for launching SCCs. The understanding 
is improved through a clear distinction between the four driver and resistor categories, consistent definitions and explanations of the influence of these categories on the SCC success. Furthermore, identification is improved, since the framework provides an extensive checklist of potential drivers and resistors for launching collaborations. To identify all relevant drivers and resistors for the considered SCC, every party should evaluate all (general) factors of the four categories with regard to their relevance. Based on the identified factors, the decision to launch the SCC can be made by evaluating whether the factors that have a positive influence on the collaboration outweigh the negative ones.

\subsubsection{Further Research Directions}

This chapter offers several opportunities for further research. The completeness of the framework was tested in the Dutch FMCG industry. Testing the framework in other geographical areas and/or industries may result in stronger support. As stated previously, the framework provides the first complete overview not only for dyadic relationships, but also for SCCs with several collaborative parties. Nevertheless, a limited sample size for each SCC was used. To build a framework for a specific type of SCC, it is necessary to conduct more interviews with parties participating in this special kind of collaboration. Furthermore, the framework should be tested in practice to discover whether it can actually close the gap between theory and practice. Therefore, it is useful to conduct surveys among parties which already participated in a failed SCC to investigate whether the SCC would either not have been launched or if failure would have been prevented if all parties involved had taken into account all factors included in the framework. Finally, this study only investigated which drivers and resistors are relevant for parties to launch an SCC, but the importance of the factors has not been quantified for the specific parties and collaborations. Quantifying the factors for each party and collaboration can be beneficial and could also be considered in further research. 


\section{A References - Observed Industries, Perspectives and SCC Types}

Table 2.7: Overview of investigated studies

\begin{tabular}{|c|c|c|c|}
\hline Reference & Industry & Perspective & SCC Type \\
\hline Akintoye et al. (2000) & Construction & Manufacturer & Vertical \\
\hline Anbanandam et al. (2011) & Apparel & Retailer & Vertical \\
\hline Barratt (2004) & - & - & - \\
\hline Beach et al. (2005) & Construction & Manufacturer & Vertical \\
\hline Boddy et al. (2000) & Electronic & $\begin{array}{c}\text { Supplier } \\
\text { Manufacturer }\end{array}$ & Vertical \\
\hline Bucklin and Sengupta (1993) & $\begin{array}{c}\text { Computer } \\
\text { Semiconductor }\end{array}$ & Manufacturer & Horizontal \\
\hline Cao and Zhang (2011) & - & Manufactuer & Vertical \\
\hline Cruijssen et al. (2007a) & Logistics & - & Horizontal \\
\hline Daugherty et al. (2006) & - & - & - \\
\hline de Leeuw and Fransoo (2009) & $\begin{array}{c}\text { Fashion } \\
\text { FMCG } \\
\text { High-tech electronics }\end{array}$ & $\begin{array}{c}\text { Manufacturer } \\
\text { Retailer }\end{array}$ & Vertical \\
\hline Ellram and Cooper (1990) & - & $\begin{array}{c}\text { Shipper } \\
\text { LSP }\end{array}$ & Vertical \\
\hline $\begin{array}{l}\text { Fawcett and Magnan (2002) } \\
\text { Fawcett et al. (2008a) } \\
\text { Fawcett et al. (2008b) } \\
\text { Fawcett et al. (2015) }\end{array}$ & - & $\begin{array}{c}\text { Retailer } \\
\text { FG assembler } \\
\text { Supplier } \\
\text { LSP } \\
\end{array}$ & Vertical \\
\hline Ganesan (1994) & - & $\begin{array}{l}\text { Supplier } \\
\text { Retailer }\end{array}$ & Vertical \\
\hline Gibson et al. (2002) & Logistics & $\begin{array}{l}\text { Shipper } \\
\text { Carrier }\end{array}$ & Vertical \\
\hline Heikkilä (2002) & Telecommunication & $\begin{array}{l}\text { Supplier } \\
\text { Customer }\end{array}$ & Vertical \\
\hline
\end{tabular}


Table 2.8: Overview of investigated studies

\begin{tabular}{|c|c|c|c|}
\hline Reference & Industry & Perspective & SCC Type \\
\hline Horvath (2001) & - & - & - \\
\hline Jharkharia and Shankar (2005) & $\begin{array}{l}\text { Automobile } \\
\text { FMCG } \\
\text { Engineering } \\
\text { Process }\end{array}$ & Manufacturer & - \\
\hline Kalwani and Narayandas (1995) & - & Supplier & Vertical \\
\hline Lambert et al. (1999) & - & - & Vertical \\
\hline Landeros et al. (1995) & - & $\begin{array}{c}\text { Buyer } \\
\text { Supplier }\end{array}$ & Vertical \\
\hline Matopoulos et al. (2007) & Agri-food & $\begin{array}{c}\text { Buyer } \\
\text { Supplier }\end{array}$ & Vertical \\
\hline Min and Zhou (2002) & - & - & - \\
\hline Min et al. (2005) & - & - & - \\
\hline Mohr and Spekman (1994) & Computer & Dealer & Vertical \\
\hline Niederkofler (1991) & - & - & - \\
\hline Perry and Sohal (2001) & $\begin{array}{l}\text { Textiles } \\
\text { Clothing } \\
\text { Footware }\end{array}$ & $\begin{array}{c}\text { Retailer } \\
\text { Manufacturer } \\
\text { Supplier }\end{array}$ & Vertical \\
\hline Reniers et al. (2010) & Chemical & - & $\begin{array}{c}\text { Vertical } \\
\text { Horizontal }\end{array}$ \\
\hline Richey Jr et al. (2010) & - & $\begin{array}{c}\text { Retailer } \\
\text { FG assembler } \\
\text { Supplier } \\
\text { Service provider }\end{array}$ & - \\
\hline Ryu et al. (2009) & - & $\begin{array}{c}\text { Buyer } \\
\text { Supplier }\end{array}$ & Vertical \\
\hline Simatupang and Sridharan (2002) & - & - & - \\
\hline Simatupang and Sridharan (2004) & - & $\begin{array}{c}\text { Supplier } \\
\text { Manufacturer }\end{array}$ & Vertical \\
\hline Walker et al. (2008) & - & Retailer & - \\
\hline Whipple and Frankel (2000) & $\begin{array}{c}\text { Food } \\
\text { Health } \\
\text { Personal care }\end{array}$ & $\begin{array}{c}\text { Buyer } \\
\text { Supplier }\end{array}$ & Vertical \\
\hline
\end{tabular}




\section{B Case Study: Enablers/Barriers}

Table 2.9: Allocation of the enablers/barriers

\begin{tabular}{|c|c|c|c|c|c|c|c|}
\hline $\begin{array}{l}\text { General } \\
\text { Factors }\end{array}$ & $\begin{array}{l}\text { Enablers/ } \\
\text { Barriers }\end{array}$ & Factors & LSP & MA & $\mathrm{RE}$ & $\mathrm{C}$ & $\mathrm{NC}$ \\
\hline \multirow[t]{2}{*}{$\begin{array}{l}\text { Presence of } \\
\text { neglectable } \\
\text { barriers }\end{array}$} & $\begin{array}{l}\text { Presence of } \\
\text { neglectable } \\
\text { barriers }\end{array}$ & $\begin{array}{l}\text { Legal } \\
\text { compliance }\end{array}$ & 1 & 1 & & 2 & \\
\hline & $\begin{array}{l}\text { Lack of absence } \\
\text { of barriers }\end{array}$ & Legal barriers & 2 & 2 & & 4 & \\
\hline $\begin{array}{l}\text { Presence of } \\
\text { neglectable } \\
\text { costs }\end{array}$ & $\begin{array}{l}\text { Lack of } \\
\text { neglectable } \\
\text { costs }\end{array}$ & $\begin{array}{l}\text { High } \\
\text { investments in } \\
\text { technology } \\
\text { SCC-specific } \\
\text { upfront } \\
\text { investments }\end{array}$ & 2 & 2 & 3 & 2 & 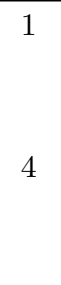 \\
\hline $\begin{array}{l}\text { Presence of } \\
\text { commitment } \\
\text { and support }\end{array}$ & $\begin{array}{l}\text { Presence of } \\
\text { commitment } \\
\text { and support }\end{array}$ & Commitment & 2 & 1 & 4 & 7 & \\
\hline \multirow[t]{2}{*}{$\begin{array}{l}\text { Presence of } \\
\text { communica- } \\
\text { tion, trans- } \\
\text { parency and } \\
\text { openness }\end{array}$} & $\begin{array}{l}\text { Presence of } \\
\text { communication, } \\
\text { transparency } \\
\text { and openness }\end{array}$ & $\begin{array}{l}\text { Honesty } \\
\text { Information } \\
\text { sharing } \\
\text { Information } \\
\text { technology } \\
\text { Transparency }\end{array}$ & $\begin{array}{l}1 \\
1 \\
3 \\
5\end{array}$ & $\begin{array}{l}2 \\
5\end{array}$ & $\begin{array}{l}2 \\
4 \\
7\end{array}$ & $\begin{array}{l}12 \\
17\end{array}$ & \\
\hline & $\begin{array}{l}\text { Lack of } \\
\text { communication, } \\
\text { transparency } \\
\text { and openness }\end{array}$ & $\begin{array}{l}\text { Difficulty to } \\
\text { share } \\
\text { confidential } \\
\text { information } \\
\text { Lack of } \\
\text { communica- } \\
\text { tion } \\
\text { Lack of } \\
\text { communica- } \\
\text { tion in } \\
\text { triangular } \\
\text { SCC }\end{array}$ & 1 & & 3 & 3 & \\
\hline
\end{tabular}


Table 2.10: Allocation of the enablers/barriers

\begin{tabular}{|c|c|c|c|c|c|c|c|}
\hline $\begin{array}{l}\text { General } \\
\text { Factors }\end{array}$ & $\begin{array}{l}\text { Enablers/ } \\
\text { Barriers }\end{array}$ & Factors & LSP & MA & $\mathrm{RE}$ & $\mathrm{C}$ & $\mathrm{NC}$ \\
\hline $\begin{array}{l}\text { Presence of } \\
\text { communica- } \\
\text { tion, trans- } \\
\text { parency and } \\
\text { openness }\end{array}$ & $\begin{array}{l}\text { Lack of com- } \\
\text { munication, } \\
\text { transparency } \\
\text { and openness }\end{array}$ & $\begin{array}{l}\text { Lack of } \\
\text { communication } \\
\text { Lack of using } \\
\text { same } \\
\text { technology }\end{array}$ & 2 & 1 & 2 & $\begin{array}{l}1 \\
5\end{array}$ & 1 \\
\hline \multirow[t]{2}{*}{$\begin{array}{l}\text { Presence of } \\
\text { compatibility }\end{array}$} & $\begin{array}{l}\text { Presence of } \\
\text { compatibility }\end{array}$ & $\begin{array}{l}\text { Common goals } \\
\text { Organizational } \\
\text { compatibility } \\
\text { Shared values } \\
\text { Strategic fit }\end{array}$ & $\begin{array}{l}4 \\
5\end{array}$ & $\begin{array}{l}1 \\
7 \\
1 \\
1\end{array}$ & $\begin{array}{l}2 \\
5\end{array}$ & $\begin{array}{c}7 \\
17 \\
\\
1 \\
5\end{array}$ & \\
\hline & $\begin{array}{l}\text { Lack of } \\
\text { compatibility }\end{array}$ & $\begin{array}{l}\text { Difficulty to } \\
\text { work with } \\
\text { competitors } \\
\text { Lack of } \\
\text { common goals } \\
\text { Lack of } \\
\text { organizational } \\
\text { compatibility } \\
\text { Power } \\
\text { imbalance }\end{array}$ & 3 & 1 & 3 & $\begin{array}{l}3 \\
18\end{array}$ & 1 \\
\hline $\begin{array}{l}\text { Presence of } \\
\text { continuous } \\
\text { improvement }\end{array}$ & $\begin{array}{l}\text { Presence of } \\
\text { continuous } \\
\text { improvement }\end{array}$ & $\begin{array}{l}\text { Continuous } \\
\text { improvement } \\
\text { of SCCs }\end{array}$ & & & 1 & 1 & \\
\hline $\begin{array}{l}\text { Presence of } \\
\text { contract }\end{array}$ & $\begin{array}{l}\text { Presence of } \\
\text { contract }\end{array}$ & $\begin{array}{l}\text { Upfront } \\
\text { agreements in } \\
\text { form of } \\
\text { contracts }\end{array}$ & & & 1 & 1 & \\
\hline $\begin{array}{l}\text { Presence of } \\
\text { experience, } \\
\text { learning and } \\
\text { knowledge }\end{array}$ & $\begin{array}{l}\text { Presence of } \\
\text { experience, } \\
\text { learning and } \\
\text { knowledge }\end{array}$ & $\begin{array}{l}\text { Step-by-step } \\
\text { approach: start } \\
\text { with smaller } \\
\text { SCCs if } \\
\text { successful } \\
\text { take next step }\end{array}$ & & & 1 & 1 & \\
\hline $\begin{array}{l}\text { Presence of } \\
\text { (fair) benefits }\end{array}$ & $\begin{array}{l}\text { Presence of } \\
\text { (fair) benefits }\end{array}$ & $\begin{array}{l}\text { Awareness of } \\
\text { possible } \\
\text { benefits }\end{array}$ & & & 1 & 1 & \\
\hline
\end{tabular}


2 A Comprehensive Framework for Holistic Evaluations of Potential Supply Chain Collaborations

Table 2.11: Allocation of the enablers/barriers

\begin{tabular}{|c|c|c|c|c|c|c|}
\hline $\begin{array}{l}\text { General } \\
\text { Factors }\end{array}$ & $\begin{array}{l}\text { Enablers/ } \\
\text { Barriers }\end{array}$ & Factors & LSP & MA & $\mathrm{RE}$ & $\mathrm{C} \quad \mathrm{NC}$ \\
\hline $\begin{array}{l}\text { Presence of } \\
\text { (fair) benefits }\end{array}$ & $\begin{array}{l}\text { Lack of (fair) } \\
\text { benefits }\end{array}$ & $\begin{array}{l}\text { Adjustment } \\
\text { of entire } \\
\text { internal } \\
\text { process } \\
\text { Difficulties in } \\
\text { establishing } \\
\text { a fair } \\
\text { allocation } \\
\text { of benefits } \\
\text { Efficiency } \\
\text { concerns }\end{array}$ & 4 & 4 & 3 & 8 \\
\hline $\begin{array}{l}\text { Presence of } \\
\text { flexibility }\end{array}$ & $\begin{array}{l}\text { Lack of } \\
\text { flexibility }\end{array}$ & $\begin{array}{l}\text { Lack of } \\
\text { flexibility }\end{array}$ & 2 & & & 2 \\
\hline $\begin{array}{l}\text { Presence of } \\
\text { goodwill }\end{array}$ & $\begin{array}{l}\text { Presence of } \\
\text { goodwill }\end{array}$ & Goodwill & & & 1 & 1 \\
\hline $\begin{array}{l}\text { Presence of } \\
\text { interdepen- } \\
\text { dence }\end{array}$ & $\begin{array}{l}\text { Presence of } \\
\text { interdependence }\end{array}$ & $\begin{array}{l}\text { Interdepend- } \\
\text { ence }\end{array}$ & 5 & 5 & 6 & 16 \\
\hline $\begin{array}{l}\text { Presence of } \\
\text { long-term } \\
\text { relationship }\end{array}$ & $\begin{array}{l}\text { Lack of } \\
\text { long-term } \\
\text { relationship }\end{array}$ & $\begin{array}{l}\text { Short-term } \\
\text { relationship }\end{array}$ & & 1 & & 1 \\
\hline \multirow[t]{2}{*}{$\begin{array}{l}\text { Presence of } \\
\text { neglecting } \\
\text { money in the } \\
\text { first meeting }\end{array}$} & $\begin{array}{l}\text { Presence of } \\
\text { neglecting } \\
\text { money in the } \\
\text { first meeting }\end{array}$ & $\begin{array}{l}\text { Neglecting } \\
\text { money in the } \\
\text { first meeting }\end{array}$ & 1 & 4 & 5 & 10 \\
\hline & $\begin{array}{l}\text { Lack of } \\
\text { neglecting } \\
\text { money in the } \\
\text { first meeting }\end{array}$ & $\begin{array}{l}\text { Difficulty to } \\
\text { speak about } \\
\text { money in the } \\
\text { first meeting }\end{array}$ & 1 & 1 & & 2 \\
\hline $\begin{array}{l}\text { Presence of } \\
\text { resources }\end{array}$ & $\begin{array}{l}\text { Lack of } \\
\text { resources }\end{array}$ & $\begin{array}{l}\text { Missing } \\
\text { capacity } \\
\text { Missing } \\
\text { employees } \\
\text { Not enough } \\
\text { time \& energy }\end{array}$ & 2 & 1 & $\begin{array}{l}1 \\
1 \\
1\end{array}$ & $\begin{array}{l}1 \\
1 \\
4\end{array}$ \\
\hline
\end{tabular}


Table 2.12: Allocation of the enablers/barriers

\begin{tabular}{|c|c|c|c|c|c|c|c|}
\hline $\begin{array}{l}\text { General } \\
\text { Factors }\end{array}$ & $\begin{array}{l}\text { Enablers/ } \\
\text { Barriers }\end{array}$ & Factors & LSP & MA & $\mathrm{RE}$ & $\mathrm{C}$ & $\mathrm{NC}$ \\
\hline \multirow[t]{2}{*}{$\begin{array}{l}\text { Presence of } \\
\text { right contact } \\
\text { person }\end{array}$} & $\begin{array}{l}\text { Presence of } \\
\text { right contact } \\
\text { person }\end{array}$ & $\begin{array}{l}\text { Relation to } \\
\text { contact } \\
\text { person }\end{array}$ & 1 & 6 & 5 & 12 & \\
\hline & $\begin{array}{l}\text { Lack of } \\
\text { right contact } \\
\text { person }\end{array}$ & $\begin{array}{l}\text { Different ways } \\
\text { of thinking of } \\
\text { contact person } \\
\text { Wrong } \\
\text { contact person }\end{array}$ & & 2 & 1 & 1 & \\
\hline $\begin{array}{l}\text { Presence of } \\
\text { time }\end{array}$ & $\begin{array}{l}\text { Presence of } \\
\text { time }\end{array}$ & $\begin{array}{l}\text { Factor time: } \\
\text { launch the } \\
\text { SCC } \\
\text { at the right } \\
\text { time }\end{array}$ & & & 1 & 1 & \\
\hline \multirow{2}{*}{$\begin{array}{l}\text { Presence of } \\
\text { trust }\end{array}$} & Presence of trust & Trust & 4 & 5 & 7 & 16 & \\
\hline & Lack of trust & Lack of trust & & & 1 & 1 & \\
\hline \multirow[t]{2}{*}{$\begin{array}{l}\text { Presence of } \\
\text { willingness } \\
\text { to change }\end{array}$} & $\begin{array}{l}\text { Presence of } \\
\text { willingness } \\
\text { to change }\end{array}$ & $\begin{array}{l}\text { Willingness } \\
\text { and drive to } \\
\text { change } \\
\text { Willingness to } \\
\text { work together } \\
\text { with different } \\
\text { parties }\end{array}$ & 1 & 1 & 1 & 2 & \\
\hline & $\begin{array}{l}\text { Lack of } \\
\text { willingness } \\
\text { to change }\end{array}$ & $\begin{array}{l}\text { Other projects } \\
\text { have higher } \\
\text { priorities }\end{array}$ & & & 2 & 1 & 1 \\
\hline
\end{tabular}




\section{C Factor Allocation}

\section{C.1 Allocation Benefits}

Table 2.13: Allocation benefits

\begin{tabular}{|c|c|c|}
\hline General Factors & Factors & Reference \\
\hline \multirow{4}{*}{$\begin{array}{l}\text { Customer } \\
\text { improvements: } \\
\text { Factors which indicate } \\
\text { improvements that } \\
\text { have a direct } \\
\text { effect on customers }\end{array}$} & $\begin{array}{l}\text { Increased customer } \\
\text { responsiveness }\end{array}$ & $\begin{array}{l}\text { Fawcett et al. (2008b); Cao and } \\
\text { Zhang (2011) }\end{array}$ \\
\hline & $\begin{array}{l}\text { Increased customer } \\
\text { satisfaction }\end{array}$ & $\begin{array}{l}\text { Fawcett et al. (2008a); Horvath } \\
(2001) \text {; Min and Zhou (2002); } \\
\text { *, MA and LSP perspective }\end{array}$ \\
\hline & $\begin{array}{l}\text { Increased delivery } \\
\text { performance }\end{array}$ & $\begin{array}{l}\text { Fawcett et al. (2008a, 2008b); } \\
\text { Horvath (2001); Lambert et al. } \\
\text { (1999); Simatupang and } \\
\text { Sridharan (2002, 2004) }\end{array}$ \\
\hline & $\begin{array}{l}\text { Improved customer } \\
\text { service }\end{array}$ & $\begin{array}{l}\text { Akintoye et al. (2000); } \\
\text { Beach et al. (2005); Cruijssen et } \\
\text { al. (2007a); Horvath (2001) }\end{array}$ \\
\hline \multirow{5}{*}{$\begin{array}{l}\text { Efficiency } \\
\text { improvements: } \\
\text { Factors which indicate } \\
\text { improvements in how } \\
\text { well expended resources } \\
\text { are utilized }\end{array}$} & $\begin{array}{l}\text { Access to missing } \\
\text { knowledge }\end{array}$ & Cao and Zhang (2011) \\
\hline & $\begin{array}{l}\text { Best value, which can } \\
\text { be drawn out of SCC } \\
\text { utilizing the specialist } \\
\text { knowledge and } \\
\text { expertise of suppliers }\end{array}$ & Beach et al. (2005) \\
\hline & Better pricing & Min et al. (2005) \\
\hline & $\begin{array}{l}\text { Closer link between } \\
\text { demand/supply }\end{array}$ & Akintoye et al. (2000) \\
\hline & $\begin{array}{l}\text { Cost reduction (e.g. } \\
\text { distribution, } \\
\text { inventory and } \\
\text { manufacturing costs) }\end{array}$ & $\begin{array}{l}\text { Kalwani and Narayandas } \\
\text { (1995); Lambert et al. (1999); } \\
\text { Min et al. (2005); Reniers et al. } \\
\text { (2010); Richey Jr et al. (2010); } \\
\text { Simatupang and Sridharan } \\
\text { (2002, 2004); Walker et al. } \\
(2008) ; *\end{array}$ \\
\hline
\end{tabular}

* refers to the case studies of Chapter 2 
Table 2.14: Allocation benefits

\begin{tabular}{|c|c|c|}
\hline General Factors & Factors & Reference \\
\hline \multirow[t]{14}{*}{$\begin{array}{l}\text { Efficiency } \\
\text { improvements }\end{array}$} & Efficiency improvement & $\begin{array}{l}\text { Cao and Zhang (2011); Horvath } \\
(2001){ }^{*}\end{array}$ \\
\hline & Employee reduction & ${ }^{*}, \mathrm{RE}$ perspective, vertical SCC \\
\hline & Firm productivity & Fawcett et al. (2008a) \\
\hline & $\begin{array}{l}\text { Focus on core } \\
\text { competencies }\end{array}$ & $\begin{array}{l}\text { Cao and Zhang (2011); Cruijssen et } \\
\text { al. (2007a); Ellram and Cooper } \\
\text { (1990), shipper perspective }\end{array}$ \\
\hline & $\begin{array}{l}\text { Gaining competitive } \\
\text { advantages }\end{array}$ & $\begin{array}{l}\text { Ellram and Cooper (1990), shipper } \\
\text { perspective; Walker et al. (2008) }\end{array}$ \\
\hline & $\begin{array}{l}\text { Improved asset } \\
\text { utilization }\end{array}$ & $\begin{array}{l}\text { Fawcett et al. (2008b); Lambert et al. } \\
\text { (1999); Min and Zhou (2002) }\end{array}$ \\
\hline & Improved expertise & $\begin{array}{l}\text { Beach et al. (2005); Ellram and } \\
\text { Cooper (1990), shipper and LSP } \\
\text { perspective }\end{array}$ \\
\hline & $\begin{array}{l}\text { Improved firm } \\
\text { performance }\end{array}$ & Richey Jr et al. (2009) \\
\hline & Improved sales & $\begin{array}{l}\text { Cao and Zhang (2011); Min et al. } \\
(2005) \text {; Simatupang and Sridharan } \\
(2002,2004)\end{array}$ \\
\hline & $\begin{array}{l}\text { Increased cash-to-cash } \\
\text { velocity }\end{array}$ & Fawcett et al. (2008b) \\
\hline & $\begin{array}{l}\text { Increased cost } \\
\text { competitiveness }\end{array}$ & Fawcett et al. (2008b) \\
\hline & Increased productivity & Fawcett et al. (2008a); Horvath (2001) \\
\hline & Increased profitability & $\begin{array}{l}\text { Akintoye et al. (2000); Cao and } \\
\text { Zhang (2011); Kalwani and Narayan- } \\
\text { das (1995); Lambert et al. (1999) }\end{array}$ \\
\hline & Increased quality & $\begin{array}{l}\text { Akintoye et al. (2000); Cao and Zhang } \\
\text { (2011); Fawcett et al. (2008b); Walker } \\
\text { et al. (2008); *, RE perspective }\end{array}$ \\
\hline
\end{tabular}


2 A Comprehensive Framework for Holistic Evaluations of Potential Supply Chain Collaborations

Table 2.15: Allocation benefits

\begin{tabular}{|c|c|c|}
\hline General Factors & Factors & Reference \\
\hline \multirow{8}{*}{$\begin{array}{l}\text { Efficiency } \\
\text { improvements }\end{array}$} & Project success & Beach et al. (2005) \\
\hline & $\begin{array}{l}\text { Rationalization and } \\
\text { simplification }\end{array}$ & Fawcett et al. (2008a, 2008b) \\
\hline & $\begin{array}{l}\text { Reducing } \\
\text { bureaucracy/paperwork }\end{array}$ & Akintoye et al. (2000) \\
\hline & $\begin{array}{l}\text { Reducing obsolete } \\
\text { inventory }\end{array}$ & $\begin{array}{l}\text { Simatupang and Sridharan (2002, } \\
2004)\end{array}$ \\
\hline & $\begin{array}{l}\text { Return on investment } \\
(\mathrm{ROI})\end{array}$ & $\begin{array}{l}\text { Cao and Zhang (2011); Min } \\
\text { et al. (2005); Min and Zhou } \\
\text { (2002); Simatupang and } \\
\text { Sridharan (2002, 2004) }\end{array}$ \\
\hline & $\begin{array}{l}\text { Streamlining product } \\
\text { flow }\end{array}$ & Perry and Sohal (2001) \\
\hline & $\begin{array}{l}\text { Streamlining supply } \\
\text { chain process }\end{array}$ & Horvath (2001); Min et al. (2005) \\
\hline & Time reduction & $\begin{array}{l}\text { Fawcett et al. (2008a, 2008b); } \\
\text { Min et al. (2005); Simatupang } \\
\text { and Sridharan (2004); * LSP } \\
\text { and RE perspective }\end{array}$ \\
\hline \multirow{6}{*}{$\begin{array}{l}\text { Enhanced } \\
\text { enablers: } \\
\text { Factors which } \\
\text { indicate improve- } \\
\text { ments in the } \\
\text { enablers through } \\
\text { SCC }\end{array}$} & Increased commitment & Min et al. (2005) \\
\hline & $\begin{array}{l}\text { Increased } \\
\text { interdependence }\end{array}$ & Min et al. (2005) \\
\hline & Increased involvement & Min et al. (2005) \\
\hline & Increased transparency & $*$, LSP perspective \\
\hline & Increased trust & $\begin{array}{l}\text { Beach et al. (2005); Min et al. } \\
(2005)\end{array}$ \\
\hline & Stronger partnership & $\begin{array}{l}\text { Beach et al. (2005); Fawcett } \\
\text { et al. (2008b); }{ }^{*}, \text { LSP } \\
\text { perspective, vertical SCC }\end{array}$ \\
\hline
\end{tabular}


Table 2.16: Allocation benefits

\begin{tabular}{|c|c|c|}
\hline General Factors & Factors & Reference \\
\hline \multirow{4}{*}{$\begin{array}{l}\text { Flexibility } \\
\text { improvements: } \\
\text { Factors which indicate } \\
\text { improvements in every } \\
\text { kind of flexibility }\end{array}$} & $\begin{array}{l}\text { Ability to handle } \\
\text { unexpected events }\end{array}$ & Fawcett et al. (2008a) \\
\hline & $\begin{array}{l}\text { Environmental } \\
\text { flexibility }\end{array}$ & Richey Jr et al. (2010) \\
\hline & Global flexibility & $\begin{array}{l}\text { Ellram and Cooper (1990), } \\
\text { shipper perspective }\end{array}$ \\
\hline & Market flexibility & $\begin{array}{l}\text { Horvath (2001); Richey Jr } \\
\text { et al. (2010) }\end{array}$ \\
\hline \multirow{2}{*}{$\begin{array}{l}\text { Image improvements: } \\
\text { Factors which indicate } \\
\text { improvements in the } \\
\text { reputation of a partner }\end{array}$} & Image improvements & $*$, MA perspective \\
\hline & $\begin{array}{l}\text { Potential for } \\
\text { receiving publicity }\end{array}$ & Walker et al. (2008) \\
\hline \multirow{8}{*}{$\begin{array}{l}\text { Market position } \\
\text { improvements: } \\
\text { Factors which indicate } \\
\text { improvements in the } \\
\text { ranking of a brand, } \\
\text { product or partner in } \\
\text { terms of its sales volume } \\
\text { relative to the sales } \\
\text { volume of its } \\
\text { market or industry }\end{array}$} & $\begin{array}{l}\text { Access new } \\
\text { markets }\end{array}$ & Fawcett et al. (2008a) \\
\hline & $\begin{array}{l}\text { Improved market } \\
\text { positioning }\end{array}$ & Reniers et al. (2010) \\
\hline & $\begin{array}{l}\text { Increase in market } \\
\text { share }\end{array}$ & $\begin{array}{l}\text { Min et al. (2005); Simatupang } \\
\text { and Sridharan }(2002,2004)\end{array}$ \\
\hline & $\begin{array}{l}\text { New product } \\
\text { development }\end{array}$ & $\begin{array}{l}\text { Horvath (2001); Lambert et al. } \\
\text { (1999); Min et al. (2005) }\end{array}$ \\
\hline & $\begin{array}{l}\text { Product quality } \\
\text { improvements }\end{array}$ & Fawcett et al. (2008a) \\
\hline & $\begin{array}{l}\text { Protecting market } \\
\text { shares }\end{array}$ & $\begin{array}{l}\text { Cruijssen et al. (2007a); } \\
\text { Lambert et al. (1999) }\end{array}$ \\
\hline & $\begin{array}{l}\text { Serving larger } \\
\text { customers }\end{array}$ & Cruijssen et al. (2007a) \\
\hline & $\begin{array}{l}\text { Unique products and } \\
\text { services }\end{array}$ & Fawcett et al. (2008b) \\
\hline
\end{tabular}


2 A Comprehensive Framework for Holistic Evaluations of Potential Supply Chain Collaborations

Table 2.17: Allocation benefits

\begin{tabular}{|c|c|c|}
\hline General Factors & Factors & Reference \\
\hline $\begin{array}{l}\text { Marketing } \\
\text { advantages: Factors } \\
\text { which indicate } \\
\text { advantages in } \\
\text { marketing }\end{array}$ & $\begin{array}{l}\text { Marketing } \\
\text { advantages }\end{array}$ & Lambert et al. (1999) \\
\hline \multirow{6}{*}{$\begin{array}{l}\text { Risk reduction: } \\
\text { Factors which indicate a } \\
\text { reduction of every kind } \\
\text { of risk }\end{array}$} & $\begin{array}{l}\text { Environmental risk } \\
\text { minimization }\end{array}$ & Walker et al. (2008) \\
\hline & $\begin{array}{l}\text { Reduced risk of } \\
\text { customer criticism }\end{array}$ & Walker et al. (2008) \\
\hline & $\begin{array}{l}\text { Reduced risk of } \\
\text { information failure }\end{array}$ & Min and Zhou (2002) \\
\hline & $\begin{array}{l}\text { Reduced risk of } \\
\text { inventory failure }\end{array}$ & Horvath (2001) \\
\hline & $\begin{array}{l}\text { Reduced risk of } \\
\text { quality failures }\end{array}$ & Min and Zhou (2002) \\
\hline & Risk reduction & Lambert et al. (1999) \\
\hline \multirow{2}{*}{$\begin{array}{l}\text { Sustainability } \\
\text { improvements: } \\
\text { Factors which indicate } \\
\text { improvements with } \\
\text { regard to sustainability }\end{array}$} & $\mathrm{CO}_{2}$ reduction & $*$ \\
\hline & Sustainability & $\begin{array}{l}\text { Walker et al. (2008); }{ }^{*}, \text { MA } \\
\text { and LSP perspective }\end{array}$ \\
\hline \multirow{2}{*}{$\begin{array}{l}\text { Technology } \\
\text { improvements: } \\
\text { Factors which indicate } \\
\text { improvements in } \\
\text { technology }\end{array}$} & $\begin{array}{l}\text { New possibilities/ } \\
\text { technologies }\end{array}$ & $*$, RE perspective \\
\hline & Technology transfers & Min and Zhou (2002) \\
\hline
\end{tabular}




\section{C.2 Allocation Forces}

Table 2.18: Allocation forces

\begin{tabular}{|c|c|c|}
\hline General Factors & Factors & Reference \\
\hline \multirow{4}{*}{$\begin{array}{l}\text { Customer issues: } \\
\text { Factors which indicate } \\
\text { that the force is based } \\
\text { on market and customer } \\
\text { demands }\end{array}$} & $\begin{array}{l}\text { Changed consumer } \\
\text { attitude }\end{array}$ & Matopoulos et al. (2007) \\
\hline & $\begin{array}{l}\text { Customer demand } \\
\text { becomes less } \\
\text { predictable }\end{array}$ & $\begin{array}{l}\text { de Leeuw and Fransoo (2009), } \\
\text { fashion, FMCG and high tech } \\
\text { electronics industry, MA and } \\
\text { RE perspective }\end{array}$ \\
\hline & $\begin{array}{l}\text { Increased supply } \\
\text { uncertainty }\end{array}$ & $\begin{array}{l}\text { de Leeuw and Fransoo (2009), } \\
\text { high tech electronics industry, } \\
\text { MA perspective }\end{array}$ \\
\hline & $\begin{array}{l}\text { More demanding } \\
\text { customers }\end{array}$ & $\begin{array}{l}\text { de Leeuw and Fransoo (2009), } \\
\text { high tech electronics industry, } \\
\text { MA perspective; Fawcett et al. } \\
\text { (2008a, 2008b); Walker et al. } \\
(2008)\end{array}$ \\
\hline \multirow{3}{*}{$\begin{array}{l}\text { Forced by other } \\
\text { parties: Factors which } \\
\text { indicate that a partner } \\
\text { and/or its surrounding } \\
\text { forced the party } \\
\text { to launch the SCC }\end{array}$} & Demanded by MA & ${ }^{*}$, LSP perspective, vertical SCC \\
\hline & Forced by RE & $\begin{array}{l}\text { de Leeuw and Fransoo (2009), } \\
\text { fashion, FMCG and high tech } \\
\text { electronics industry, MA } \\
\text { perspective; *, MA and LSP } \\
\text { perspective, vertical SCC }\end{array}$ \\
\hline & $\begin{array}{l}\text { Regulatory } \\
\text { compliance }\end{array}$ & $\begin{array}{l}\text { Matopoulos et al. (2007); } \\
\text { Walker et al. (2008) }\end{array}$ \\
\hline \multirow{2}{*}{$\begin{array}{l}\text { Globalization: Factors } \\
\text { which indicate that a party } \\
\text { is forced by worldwide } \\
\text { movements towards trade, } \\
\text { economic, financial and } \\
\text { communication integration }\end{array}$} & $\begin{array}{l}\text { Economic } \\
\text { globalization }\end{array}$ & Fawcett et al. (2008a, 2008b) \\
\hline & Globalization & Matopoulos et al. (2007) \\
\hline
\end{tabular}


Table 2.19: Allocation forces

\begin{tabular}{|c|c|c|}
\hline General Factors & Factors & Reference \\
\hline $\begin{array}{l}\text { Greater competitive } \\
\text { intensity: Factors } \\
\text { which indicate that the } \\
\text { force is based on } \\
\text { competitiveness issues }\end{array}$ & $\begin{array}{l}\text { Greater competitive } \\
\text { intensity }\end{array}$ & $\begin{array}{l}\text { Fawcett et al. (2008a, 2008b); } \\
\text { Matopoulos et al. (2007) }\end{array}$ \\
\hline \multirow{2}{*}{$\begin{array}{l}\text { Information issues: } \\
\text { Factors which indicate } \\
\text { that the force is based } \\
\text { on information issues }\end{array}$} & $\begin{array}{l}\text { Information } \\
\text { revolution }\end{array}$ & Fawcett et al. (2008b) \\
\hline & $\begin{array}{l}\text { Need for better } \\
\text { information }\end{array}$ & Fawcett et al. (2008a) \\
\hline \multirow{2}{*}{$\begin{array}{l}\text { Monetary issues: } \\
\text { Factors which indicate } \\
\text { that the force is based } \\
\text { on monetary issues }\end{array}$} & $\begin{array}{l}\text { Increased financial } \\
\text { pressure }\end{array}$ & Fawcett et al. (2008b) \\
\hline & $\begin{array}{l}\text { Pressure for lower } \\
\text { prices }\end{array}$ & Walker et al. (2008) \\
\hline \multirow[t]{2}{*}{$\begin{array}{l}\text { Product issues: } \\
\text { Factors which indicate } \\
\text { that the force is based } \\
\text { on product issues }\end{array}$} & Critical product & $\begin{array}{l}\text { de Leeuw and Fransoo (2009), } \\
\text { fashion, FMCG and high tech } \\
\text { electronics industry, MA and } \\
\text { RE perspective }\end{array}$ \\
\hline & $\begin{array}{l}\text { Product } \\
\text { customization }\end{array}$ & $\begin{array}{l}\text { de Leeuw and Fransoo (2009), } \\
\text { FMCG and high tech } \\
\text { electronics industry, MA and } \\
\text { RE perspective }\end{array}$ \\
\hline \multirow{2}{*}{$\begin{array}{l}\text { Shifting issues: Factors } \\
\text { which indicate that the } \\
\text { force is based on changes } \\
\text { in persons, configurations } \\
\text { or focuses }\end{array}$} & $\begin{array}{l}\text { Shifting channel } \\
\text { power }\end{array}$ & Fawcett et al. (2008a, 2008b) \\
\hline & $\begin{array}{l}\text { Shifting competitive } \\
\text { focus }\end{array}$ & Fawcett et al. (2008a, 2008b) \\
\hline \multirow{2}{*}{$\begin{array}{l}\text { Technology issues: } \\
\text { Factors which indicate } \\
\text { that the force is based } \\
\text { on technology issues }\end{array}$} & $\begin{array}{l}\text { New information } \\
\text { technology }\end{array}$ & Fawcett et al. (2008a) \\
\hline & $\begin{array}{l}\text { Rapid technological } \\
\text { change }\end{array}$ & Bucklin and Sengupta (1993) \\
\hline
\end{tabular}


Table 2.20: Allocation forces

\begin{tabular}{l|l|l}
\hline General Factors & Factors & Reference \\
\hline \hline Tighter relationships: & Merge and & Fawcett et al. (2008a, 2008b) \\
Factors which indicate that & $\begin{array}{l}\text { acquisition activities } \\
\text { the force is based on }\end{array}$ & \\
\cline { 2 - 3 } changes in relationships & Tighter alliance & Fawcett et al. (2008a) \\
\hline Time issues: Factors & relationships & \\
which indicate that the & Compressed product & Fawcett et al. (2008a); \\
force is based on time & cycles & Richey Jr et al. (2009) \\
\cline { 2 - 3 } issues & Compressed & Fawcett et al. (2008b) \\
\hline
\end{tabular}




\section{C.3 Allocation Enablers/Barriers}

Table 2.21: Allocation enablers/barriers

\begin{tabular}{|c|c|c|c|}
\hline General Factors & Enablers/ Barriers & Factors & Reference \\
\hline \multirow{7}{*}{$\begin{array}{l}\text { Presence of } \\
\text { neglectable } \\
\text { barriers: } \\
\text { Factors } \\
\text { which } \\
\text { indicate the } \\
\text { presence or } \\
\text { lack of } \\
\text { neglectable } \\
\text { legal, } \\
\text { industrial } \\
\text { or organiza- } \\
\text { tional } \\
\text { barriers }\end{array}$} & \multirow[t]{2}{*}{$\begin{array}{l}\text { Presence of } \\
\text { neglectable } \\
\text { barriers }\end{array}$} & $\begin{array}{l}\text { Legal } \\
\text { compliance }\end{array}$ & $\begin{array}{l}*, \text { MA and LSP } \\
\text { perspective, vertical } \\
\text { SCC }\end{array}$ \\
\hline & & $\begin{array}{l}\text { No organiza- } \\
\text { tional barriers }\end{array}$ & Walker et al. (2008) \\
\hline & \multirow[t]{5}{*}{$\begin{array}{l}\text { Lack of } \\
\text { neglectable } \\
\text { barriers }\end{array}$} & $\begin{array}{l}\text { Distinguish one- } \\
\text { self towards the } \\
\text { coalition } \\
\text { partners in a } \\
\text { horizontal SCC }\end{array}$ & Cruijssen et al. (2007a) \\
\hline & & $\begin{array}{l}\text { Industry } \\
\text { specific } \\
\text { barriers }\end{array}$ & $\begin{array}{l}\text { Matopoulos et al. (2007); } \\
\text { Walker et al. (2008) }\end{array}$ \\
\hline & & Legal barriers & $\begin{array}{l}*, \text { LSP and MA } \\
\text { perspective, horizontal } \\
\text { SCC }\end{array}$ \\
\hline & & $\begin{array}{l}\text { Organizational } \\
\text { boundaries (e.g. } \\
\text { culture barriers) }\end{array}$ & $\begin{array}{l}\text { Akintoye et al. (2000); } \\
\text { Fawcett et al. (2008a); } \\
\text { Matopoulos et al. } \\
(2007) \text {; Walker et al. } \\
(2008)\end{array}$ \\
\hline & & $\begin{array}{l}\text { Conflicting } \\
\text { goals and/ or } \\
\text { priorities }\end{array}$ & Walker et al. (2008) \\
\hline
\end{tabular}


Table 2.22: Allocation enablers/barriers

\begin{tabular}{|c|c|c|c|}
\hline General Factors & $\begin{array}{l}\text { Enablers/ } \\
\text { Barriers }\end{array}$ & Factors & Reference \\
\hline \multirow{3}{*}{$\begin{array}{l}\text { Presence of } \\
\text { neglectable } \\
\text { costs: Factors } \\
\text { which indicate } \\
\text { the presence } \\
\text { or lack of } \\
\text { neglectable costs }\end{array}$} & \multirow[t]{3}{*}{$\begin{array}{l}\text { Lack of } \\
\text { neglectable } \\
\text { costs }\end{array}$} & $\begin{array}{l}\text { High } \\
\text { investments } \\
\text { in technology }\end{array}$ & $\begin{array}{l}\text { Cruijssen et al. }(2007 a) ;{ }^{*} \\
\text { LSP and RE perspective }\end{array}$ \\
\hline & & Increased costs & Walker et al. (2008) \\
\hline & & $\begin{array}{l}\text { Relationship- } \\
\text { specific upfront } \\
\text { investments }\end{array}$ & $\begin{array}{l}\text { Beach et al. (2005); } \\
\text { Lambert et al. (1996); Min } \\
\text { and Zhou (2002); Reniers } \\
\text { et al. (2010); * }\end{array}$ \\
\hline $\begin{array}{l}\text { Presence of } \\
\text { accountabil- } \\
\text { ity: Factors } \\
\text { which indicate } \\
\text { the presence } \\
\text { or lack of } \\
\text { obligation } \\
\text { of a partner }\end{array}$ & $\begin{array}{l}\text { Presence of } \\
\text { account- } \\
\text { ability }\end{array}$ & $\begin{array}{l}\text { Clarity of } \\
\text { accountability }\end{array}$ & Riggin et al. (1992) \\
\hline \multirow{3}{*}{$\begin{array}{l}\text { Presence of } \\
\text { appropriate } \\
\text { type of SCC: } \\
\text { Factors which } \\
\text { indicate the } \\
\text { presence or } \\
\text { lack of the } \\
\text { appropriate } \\
\text { type of SCC }\end{array}$} & \multirow[t]{3}{*}{$\begin{array}{l}\text { Presence of } \\
\text { appropriate } \\
\text { type of SCC }\end{array}$} & $\begin{array}{l}\text { Define an } \\
\text { appropriate } \\
\text { type of SCC } \\
\text { to establish } \\
\text { with supply } \\
\text { chain partners }\end{array}$ & Fawcett et al. (2008b) \\
\hline & & $\begin{array}{l}\text { Scale of the } \\
\text { SCC }\end{array}$ & Walker et al. (2008) \\
\hline & & SCC Scope & Lambert et al. (1999) \\
\hline \multirow{3}{*}{$\begin{array}{l}\text { Presence of } \\
\text { capabilities: } \\
\text { Factors which } \\
\text { indicate the } \\
\text { presence or } \\
\text { lack of an } \\
\text { ability }\end{array}$} & \multirow[t]{2}{*}{$\begin{array}{l}\text { Presence of } \\
\text { capabilities }\end{array}$} & $\begin{array}{l}\text { Ability to meet } \\
\text { (performance) } \\
\text { expectation }\end{array}$ & Whipple and Frankel (2000) \\
\hline & & $\begin{array}{l}\text { Partner } \\
\text { capabilities }\end{array}$ & $\begin{array}{l}\text { de Leeuw and Fransoo } \\
(2009) \text {, fashion, FMCG and } \\
\text { high tech electronics } \\
\text { industry, MA and RE } \\
\text { perspective }\end{array}$ \\
\hline & $\begin{array}{l}\text { Lack of } \\
\text { capabilities }\end{array}$ & $\begin{array}{l}\text { Lack of partner } \\
\text { capabilities }\end{array}$ & $\begin{array}{l}\text { Jharkharia and Shankar } \\
(2005)\end{array}$ \\
\hline
\end{tabular}


Table 2.23: Allocation enablers/barriers

\begin{tabular}{|c|c|c|c|}
\hline General Factors & $\begin{array}{l}\text { Enablers/ } \\
\text { Barriers }\end{array}$ & Factors & Reference \\
\hline \multirow{4}{*}{$\begin{array}{l}\text { Presence of } \\
\text { commitment } \\
\text { and support: } \\
\text { Factors which } \\
\text { indicate the } \\
\text { presence or } \\
\text { lack of } \\
\text { commitment } \\
\text { and/or } \\
\text { support of } \\
\text { parties and } \\
\text { their } \\
\text { surroundings }\end{array}$} & \multirow[t]{2}{*}{$\begin{array}{l}\text { Presence of } \\
\text { commitment } \\
\text { and support }\end{array}$} & Commitment & $\begin{array}{l}\text { Anbanandam et al. (2011); } \\
\text { Barratt (2004); Beach et al. } \\
(2005) \text {; Fawcett et al. } \\
(2008 b) ; \text { Heikkilä (2002); } \\
\text { Lambert et al. (1999); } \\
\text { Mohr and Spekman (1994); } \\
\text { Perry and Sohal (2001); } \\
\text { Ryu et al. (2009); * }\end{array}$ \\
\hline & & Support & $\begin{array}{l}\text { Barratt (2004); Niederkofler } \\
\text { (1991); Whipple and } \\
\text { Frankel }(2000)\end{array}$ \\
\hline & \multirow{2}{*}{$\begin{array}{l}\text { Lack of } \\
\text { commitment } \\
\text { and support }\end{array}$} & $\begin{array}{l}\text { Lack of } \\
\text { commitment }\end{array}$ & Akintoye et al. (2000) \\
\hline & & $\begin{array}{l}\text { Lack of } \\
\text { support }\end{array}$ & $\begin{array}{l}\text { Fawcett et al. (2008b); } \\
\text { Lambert et al. (1999); } \\
\text { Niederkofler (1991) }\end{array}$ \\
\hline \multirow{3}{*}{$\begin{array}{l}\text { Presence of } \\
\text { communica- } \\
\text { tion, trans- } \\
\text { parency and } \\
\text { openness: } \\
\text { Factors which } \\
\text { indicate the } \\
\text { presence or } \\
\text { lack of formal } \\
\text { and informal } \\
\text { information } \\
\text { sharing with } \\
\text { the parties in } \\
\text { a timely and } \\
\text { qualitative } \\
\text { manner } \\
\text { and in an } \\
\text { appropriate way }\end{array}$} & \multirow[t]{3}{*}{$\begin{array}{l}\text { Presence of } \\
\text { communication, } \\
\text { transparency } \\
\text { and openness }\end{array}$} & $\begin{array}{l}\text { Communica- } \\
\text { tion }\end{array}$ & $\begin{array}{l}\text { Barratt (2004); Beach et al. } \\
(2005) \text {; Cao and Zhang } \\
(2011) \text {; Fawcett et al. } \\
(2008 a) \text {; Heikkilä (2002); } \\
\text { Lambert et al. (1999); Min } \\
\text { and Zhou (2002); Min et al. } \\
(2005) \text {; Perry and Sohal } \\
(2001) \text {; Ryu et al. (2009) }\end{array}$ \\
\hline & & $\begin{array}{l}\text { Communica- } \\
\text { tion behavior }\end{array}$ & Mohr and Spekman (1994) \\
\hline & & $\begin{array}{l}\text { Communica- } \\
\text { tion quality }\end{array}$ & $\begin{array}{l}\text { Heikkilä (2002); Mohr and } \\
\text { Spekman (1994); } \\
\text { Niederkofler (1991) }\end{array}$ \\
\hline
\end{tabular}


Table 2.24: Allocation enablers/barriers

\begin{tabular}{|c|c|c|c|}
\hline General Factors & $\begin{array}{l}\text { Enablers/ } \\
\text { Barriers }\end{array}$ & Factors & Reference \\
\hline \multirow[t]{8}{*}{$\begin{array}{l}\text { Presence of } \\
\text { communica- } \\
\text { tion, } \\
\text { transparency } \\
\text { and openness }\end{array}$} & \multirow[t]{6}{*}{$\begin{array}{l}\text { Presence of } \\
\text { communica- } \\
\text { tion, } \\
\text { transparency } \\
\text { and openness }\end{array}$} & $\begin{array}{l}\text { Free flow of } \\
\text { information }\end{array}$ & $\begin{array}{l}\text { Akintoye et al. (2000); } \\
\text { Min et al. (2005); Perry } \\
\text { and Sohal (2001) }\end{array}$ \\
\hline & & Honesty & $\begin{array}{l}\text { Barratt (2004); Heikkilä } \\
(2002) ;{ }^{*}, \text { LSP } \\
\text { perspective }\end{array}$ \\
\hline & & $\begin{array}{l}\text { Information } \\
\text { sharing }\end{array}$ & $\begin{array}{l}\text { Anbanandam et al. } \\
\text { (2011); Barratt (2004); } \\
\text { Cao and Zhang (2011); } \\
\text { Fawcett et al. (2008a, } \\
\text { 2008b); Gibson et al. } \\
\text { (2002); Heikkilä (2002); } \\
\text { Perry and Sohal (2001); } \\
\text { Richey Jr et al. (2010); } \\
\text { Simatupang and } \\
\text { Sridharan (2002); * }\end{array}$ \\
\hline & & $\begin{array}{l}\text { Information } \\
\text { technology }\end{array}$ & $\begin{array}{l}\text { Akintoye et al. (2000); } \\
\text { Barratt (2004); Boddy } \\
\text { et al. (2000); Fawcett et } \\
\text { al. (2008a, 2008b); } \\
\text { Horvath (2001); Perry } \\
\text { and Sohal (2001); * }\end{array}$ \\
\hline & & Openness & $\begin{array}{l}\text { Barratt (2004); Heikkilä } \\
(2002) \text {; Reniers et al. } \\
(2010)\end{array}$ \\
\hline & & Transparency & $*$ \\
\hline & \multirow{2}{*}{$\begin{array}{l}\text { Lack of } \\
\text { communica- } \\
\text { tion, } \\
\text { transparency } \\
\text { and openness }\end{array}$} & $\begin{array}{l}\text { Difficulty to } \\
\text { share confidential } \\
\text { information }\end{array}$ & $*$, LSP perspective \\
\hline & & $\begin{array}{l}\text { Lack of } \\
\text { (appropriate) } \\
\text { information } \\
\text { technology }\end{array}$ & $\begin{array}{l}\text { Akintoye et al. (2000); } \\
\text { Fawcett et al. (2008a); } \\
\text { Fawcett et al. (2015); } \\
\text { Jharkharia and Shankar } \\
(2005)\end{array}$ \\
\hline
\end{tabular}


2 A Comprehensive Framework for Holistic Evaluations of Potential Supply Chain Collaborations

Table 2.25: Allocation enablers/barriers

\begin{tabular}{|c|c|c|c|}
\hline $\begin{array}{l}\text { General } \\
\text { Factors }\end{array}$ & $\begin{array}{l}\text { Enablers/ } \\
\text { Barriers }\end{array}$ & Factors & Reference \\
\hline \multirow{8}{*}{$\begin{array}{l}\text { Presence of } \\
\text { communica- } \\
\text { tion, } \\
\text { transparency } \\
\text { and openness }\end{array}$} & \multirow{8}{*}{$\begin{array}{l}\text { Lack of } \\
\text { communica- } \\
\text { tion, } \\
\text { transparency } \\
\text { and openness }\end{array}$} & $\begin{array}{l}\text { Lack of } \\
\text { availability, } \\
\text { accessibility and } \\
\text { validity of data }\end{array}$ & Riggin et al. (1992) \\
\hline & & $\begin{array}{l}\text { Lack of } \\
\text { communication }\end{array}$ & *, LSP perspective \\
\hline & & $\begin{array}{l}\text { Lack of communi- } \\
\text { cation between RE } \\
\text { and LSP in trian- } \\
\text { gular relationships }\end{array}$ & ${ }^{*}$, RE perspective \\
\hline & & $\begin{array}{l}\text { Lack of } \\
\text { information } \\
\text { sharing }\end{array}$ & $\begin{array}{l}\text { Fawcett et al. (2008a, } \\
\text { 2008b); Fawcett et al. } \\
\text { (2015); Lambert et al. } \\
\text { (1999); Simatupang and } \\
\text { Sridharan (2002); } \\
\text { Richey Jr et al. (2010); } \\
\text { Walker et al. (2008) }\end{array}$ \\
\hline & & $\begin{array}{l}\text { Lack of } \\
\text { transparency }\end{array}$ & $\begin{array}{l}\text { Fawcett and Magnan } \\
(2002) ; * \text {, MA } \\
\text { perspective }\end{array}$ \\
\hline & & $\begin{array}{l}\text { Lack of using the } \\
\text { same technology }\end{array}$ & $*$ \\
\hline & & $\begin{array}{l}\text { No implementation } \\
\text { issues addressed } \\
\text { during negotiations }\end{array}$ & Niederkofler (1991) \\
\hline & & $\begin{array}{l}\text { Very complex ICT } \\
\text { system }\end{array}$ & *, LSP perspective \\
\hline
\end{tabular}


Table 2.26: Allocation enablers/barriers

\begin{tabular}{|c|c|c|c|}
\hline $\begin{array}{l}\text { General } \\
\text { Factors }\end{array}$ & $\begin{array}{l}\text { Enablers/ } \\
\text { Barriers }\end{array}$ & Factors & Reference \\
\hline \multirow{15}{*}{$\begin{array}{l}\text { Presence of } \\
\text { compatibil- } \\
\text { ity: } \\
\text { Factors which } \\
\text { indicate the } \\
\text { presence or } \\
\text { lack of } \\
\text { complementar- } \\
\text { ties } \\
\text { between the } \\
\text { partners in } \\
\text { terms of e.g. } \\
\text { goals and } \\
\text { cultures }\end{array}$} & \multirow[t]{15}{*}{$\begin{array}{l}\text { Presence of } \\
\text { compatibility }\end{array}$} & $\begin{array}{l}\text { Business process } \\
\text { compatibility }\end{array}$ & $\begin{array}{l}\text { Barratt (2004); Boddy } \\
\text { et al. }(2000)\end{array}$ \\
\hline & & $\begin{array}{l}\text { Common/clear } \\
\text { goals }\end{array}$ & $\begin{array}{l}\text { Cao and Zhang (2011); } \\
\text { Fawcett et al. (2008a); } \\
\text { Whipple and Frankel } \\
(2000) ; *\end{array}$ \\
\hline & & $\begin{array}{l}\text { Common } \\
\text { interests }\end{array}$ & Akintoye et al. (2000) \\
\hline & & $\begin{array}{l}\text { Common operat- } \\
\text { ing procedures }\end{array}$ & Fawcett et al. (2008a) \\
\hline & & Common vision & $\begin{array}{l}\text { Beach et al. (2005); Fawcett } \\
\text { et al. (2008b) }\end{array}$ \\
\hline & & $\begin{array}{l}\text { Compatibility in } \\
\text { structure }\end{array}$ & Boddy et al. (2000) \\
\hline & & $\begin{array}{l}\text { Corporate } \\
\text { compatibility }\end{array}$ & Lambert et al. (1999) \\
\hline & & $\begin{array}{l}\text { Cultural } \\
\text { compatibility }\end{array}$ & $\begin{array}{l}\text { Boddy et al. (2000); Reniers } \\
\text { et al. (2010) }\end{array}$ \\
\hline & & $\begin{array}{l}\text { Decision } \\
\text { synchronization }\end{array}$ & $\begin{array}{l}\text { Cao and Zhang (2011); } \\
\text { Simatupang and Sridharan } \\
(2002)\end{array}$ \\
\hline & & Equal power & Gibson et al. (2002) \\
\hline & & Exclusivity & Lambert et al. (1999) \\
\hline & & $\begin{array}{l}\text { Incentive } \\
\text { alignment }\end{array}$ & $\begin{array}{l}\text { Cao and Zhang (2011); } \\
\text { Simatupang and Sridharan } \\
(2002)\end{array}$ \\
\hline & & $\begin{array}{l}\text { Internal } \\
\text { alignment }\end{array}$ & Min et al. (2005) \\
\hline & & $\begin{array}{l}\text { Level of being } \\
\text { supplementary/ } \\
\text { complementary }\end{array}$ & Reniers et al. (2010) \\
\hline & & $\begin{array}{l}\text { Management } \\
\text { compatibility }\end{array}$ & Lambert et al. (1999) \\
\hline
\end{tabular}


2 A Comprehensive Framework for Holistic Evaluations of Potential Supply Chain Collaborations

Table 2.27: Allocation enablers/barriers

\begin{tabular}{|c|c|c|c|}
\hline $\begin{array}{l}\text { General } \\
\text { Factors }\end{array}$ & $\begin{array}{l}\text { Enablers/ } \\
\text { Barriers }\end{array}$ & Factors & Reference \\
\hline \multirow[t]{13}{*}{$\begin{array}{l}\text { Presence of } \\
\text { compatibil- } \\
\text { ity }\end{array}$} & \multirow[t]{13}{*}{$\begin{array}{l}\text { Presence of } \\
\text { compatibility }\end{array}$} & $\begin{array}{l}\text { Objective } \\
\text { alignment }\end{array}$ & $\begin{array}{l}\text { Beach et al. (2005); Fawcett } \\
\text { et al. (2008b); Simatupang } \\
\text { and Sridharan (2002) }\end{array}$ \\
\hline & & $\begin{array}{l}\text { Operational } \\
\text { compatibility }\end{array}$ & $\begin{array}{l}\text { Niederkofler (1991); Ryu } \\
\text { et al. (2009) }\end{array}$ \\
\hline & & $\begin{array}{l}\text { Organizational } \\
\text { compatibility }\end{array}$ & $\begin{array}{l}\text { Bucklin and Sengupta (1993); } \\
\text { Richey Jr et al. (2010); }{ }^{*}\end{array}$ \\
\hline & & $\begin{array}{l}\text { Partner } \\
\text { compatibility }\end{array}$ & $\begin{array}{l}\text { Whipple and Frankel (2000); } \\
\text { Fawcett et al. (2008a, 2008b); } \\
\text { Reniers et al. (2010) }\end{array}$ \\
\hline & & $\begin{array}{l}\text { People/employee } \\
\text { compatibility }\end{array}$ & Boddy et al. (2000) \\
\hline & & $\begin{array}{l}\text { Physical } \\
\text { proximity }\end{array}$ & Lambert et al. (1999) \\
\hline & & $\begin{array}{l}\text { Prior history of } \\
\text { working together } \\
\text { with the partner }\end{array}$ & $\begin{array}{l}\text { Bucklin and Sengupta (1993); } \\
\text { Lambert et al. (1999) }\end{array}$ \\
\hline & & $\begin{array}{l}\text { Shared } \\
\text { competitors }\end{array}$ & Lambert et al. (1999) \\
\hline & & $\begin{array}{l}\text { Shared high } \\
\text { value } \\
\text { end users }\end{array}$ & Lambert et al. (1999) \\
\hline & & Shared values & $\begin{array}{l}* \text {, MA perspective, vertical } \\
\text { SCC }\end{array}$ \\
\hline & & Strategic fit & $\begin{array}{l}\text { Gibson et al. (2002); } \\
\text { Niederkofler (1991); Ryu et al } \\
(2009){ }^{*}\end{array}$ \\
\hline & & $\begin{array}{l}\text { Symmetry of the } \\
\text { parties; parties } \\
\text { benefit from } \\
\text { each } \\
\text { other }\end{array}$ & Lambert et al. (1999) \\
\hline & & VMI & Fawcett et al. (2008a) \\
\hline
\end{tabular}


Table 2.28: Allocation enablers/barriers

\begin{tabular}{|c|c|c|c|}
\hline $\begin{array}{l}\text { General } \\
\text { Factors }\end{array}$ & $\begin{array}{l}\text { Enablers/ } \\
\text { Barriers }\end{array}$ & Factors & Reference \\
\hline \multirow{12}{*}{$\begin{array}{l}\text { Presence } \\
\text { of compa- } \\
\text { tibility }\end{array}$} & \multirow[t]{12}{*}{$\begin{array}{l}\text { Lack of } \\
\text { compatibility }\end{array}$} & $\begin{array}{l}\text { Cultural } \\
\text { differences }\end{array}$ & Lambert et al. (1999) \\
\hline & & $\begin{array}{l}\text { Difficulty to } \\
\text { work with } \\
\text { competitors }\end{array}$ & $\begin{array}{l}\text { *, MA perspective, horizontal } \\
\text { SCC }\end{array}$ \\
\hline & & $\begin{array}{l}\text { Disagreement } \\
\text { over } \\
\text { the domain of } \\
\text { decision }\end{array}$ & $\begin{array}{l}\text { Simatupang and Sridharan } \\
(2002)\end{array}$ \\
\hline & & $\begin{array}{l}\text { Firms' way of } \\
\text { managing their } \\
\text { businesses are } \\
\text { different and } \\
\text { clash }\end{array}$ & Niederkofler (1991) \\
\hline & & $\begin{array}{l}\text { Incentive } \\
\text { misalignment }\end{array}$ & $\begin{array}{l}\text { Simatupang and Sridharan } \\
(2002)\end{array}$ \\
\hline & & $\begin{array}{l}\text { Inconsistent } \\
\text { operating goal }\end{array}$ & Fawcett et al. (2008a) \\
\hline & & $\begin{array}{l}\text { Lack of common } \\
\text { goals }\end{array}$ & $\begin{array}{l}\text { Lambert et al. (1999); } \\
\text { Richey Jr et al. (2010); }{ }^{*}, \mathrm{RE} \\
\text { perspective }\end{array}$ \\
\hline & & $\begin{array}{l}\text { Lack of } \\
\text { organizational } \\
\text { compatibility }\end{array}$ & Richey Jr et al. (2010); * \\
\hline & & $\begin{array}{l}\text { Managerial } \\
\text { resource } \\
\text { imbalance }\end{array}$ & Bucklin and Sengupta (1993) \\
\hline & & No strategic fit & $\begin{array}{l}\text { Fawcett et al. (2008b); Fawcett } \\
\text { et al. (2015); Lambert et al. } \\
\text { (1999); Niederkofler (1991) }\end{array}$ \\
\hline & & $\begin{array}{l}\text { Objective } \\
\text { differences }\end{array}$ & $\begin{array}{l}\text { Simatupang and Sridharan } \\
(2002)\end{array}$ \\
\hline & & Operating misfit & $\begin{array}{l}\text { Fawcett et al. (2008b); } \\
\text { Niederkofler (1991) }\end{array}$ \\
\hline
\end{tabular}


2 A Comprehensive Framework for Holistic Evaluations of Potential Supply Chain Collaborations

Table 2.29: Allocation enablers/barriers

\begin{tabular}{|c|c|c|c|}
\hline General Factors & $\begin{array}{l}\text { Enablers/ } \\
\text { Barriers }\end{array}$ & Factors & Reference \\
\hline \multirow[t]{2}{*}{$\begin{array}{l}\text { Presence of } \\
\text { compatibility }\end{array}$} & \multirow[t]{2}{*}{$\begin{array}{l}\text { Lack of } \\
\text { compatibility }\end{array}$} & $\begin{array}{l}\text { Power } \\
\text { imbalance }\end{array}$ & $\begin{array}{l}\text { Boddy et al. (2000); } \\
\text { Bucklin and Sengupta } \\
(1993) ; \text { Cruijssen et al. } \\
(2007 \text { a); Lambert et al. } \\
(1999) ; \text { Matopoulos et al. } \\
\text { (2007); * MA perspective, } \\
\text { vertical SCC }\end{array}$ \\
\hline & & $\begin{array}{l}\text { Unrealistic } \\
\text { expectations }\end{array}$ & Lambert et al. (1999) \\
\hline $\begin{array}{l}\text { Presence of } \\
\text { confidential- } \\
\text { ity: } \\
\text { Factors which } \\
\text { indicate the } \\
\text { presence or } \\
\text { lack of } \\
\text { exchanging } \\
\text { proprietary } \\
\text { information }\end{array}$ & $\begin{array}{l}\text { Lack of } \\
\text { confidentiality }\end{array}$ & $\begin{array}{l}\text { Lack of } \\
\text { confidential- } \\
\text { ity }\end{array}$ & Walker et al. (2008) \\
\hline \multirow{2}{*}{$\begin{array}{l}\text { Presence of } \\
\text { continuous } \\
\text { improvement: } \\
\text { Factors which } \\
\text { indicate the } \\
\text { presence or } \\
\text { lack of } \\
\text { continuous } \\
\text { improvement }\end{array}$} & \multirow[t]{2}{*}{$\begin{array}{l}\text { Presence of } \\
\text { continuous } \\
\text { improvement }\end{array}$} & $\begin{array}{l}\text { Continuous } \\
\text { performance } \\
\text { evaluation/ } \\
\text { improvement }\end{array}$ & $\begin{array}{l}\text { Beach et al. (2005); Gibson } \\
\text { et al. (2002) }\end{array}$ \\
\hline & & $\begin{array}{l}\text { Continuous } \\
\text { improvement } \\
\text { of SCCs }\end{array}$ & *, RE perspective \\
\hline
\end{tabular}


Table 2.30: Allocation enablers/barriers

\begin{tabular}{|c|c|c|c|}
\hline $\begin{array}{l}\text { General } \\
\text { Factors }\end{array}$ & $\begin{array}{l}\text { Enablers/ } \\
\text { Barriers }\end{array}$ & Factors & Reference \\
\hline \multirow{5}{*}{$\begin{array}{l}\text { Presence of } \\
\text { contract: } \\
\text { Factors which } \\
\text { indicate the } \\
\text { presence or } \\
\text { lack of } \\
\text { agreements } \\
\text { between the } \\
\text { partners }\end{array}$} & \multirow[t]{4}{*}{$\begin{array}{l}\text { Presence of } \\
\text { contract }\end{array}$} & Clear guidelines & $\begin{array}{l}\text { Fawcett et al. (2008a); } \\
\text { Gibson et al. (2002); } \\
\text { Niederkofler (1991); } \\
\text { Richey Jr et al. (2010) }\end{array}$ \\
\hline & & Formalization & $\begin{array}{l}\text { Daugherty et al. (2006); } \\
\text { Min et al. (2005) }\end{array}$ \\
\hline & & Right contract style & Lambert et al. (1999) \\
\hline & & $\begin{array}{l}\text { Upfront agreements } \\
\text { in form of contracts }\end{array}$ & $\begin{array}{l}*, \text { RE perspective, } \\
\text { horizontal and vertical SCC }\end{array}$ \\
\hline & $\begin{array}{l}\text { Lack of } \\
\text { contract }\end{array}$ & $\begin{array}{l}\text { Lack of alliance } \\
\text { guidelines }\end{array}$ & $\begin{array}{l}\text { Fawcett et al. (2008a); } \\
\text { Richey Jr et al. (2010) }\end{array}$ \\
\hline \multirow{2}{*}{$\begin{array}{l}\text { Presence of } \\
\text { control: } \\
\text { Factors which } \\
\text { indicate the } \\
\text { presence or } \\
\text { lack of control }\end{array}$} & $\begin{array}{l}\text { Presence of } \\
\text { control }\end{array}$ & $\begin{array}{l}\text { Joint operating } \\
\text { controls }\end{array}$ & Lambert et al. (1999) \\
\hline & $\begin{array}{l}\text { Lack of } \\
\text { control }\end{array}$ & Loss of control & $\begin{array}{l}\text { Ellram and Cooper (1990), } \\
\text { shipper perspective; } \\
\text { Lambert et al. (1999) }\end{array}$ \\
\hline $\begin{array}{l}\text { Presence of } \\
\text { coordination: } \\
\text { Factors which } \\
\text { indicate the } \\
\text { presence or } \\
\text { lack of } \\
\text { synchronization } \\
\text { and integrating }\end{array}$ & $\begin{array}{l}\text { Presence of } \\
\text { coordina- } \\
\text { tion }\end{array}$ & Coordination & $\begin{array}{l}\text { Mohr and Spekman (1994); } \\
\text { Riggin et al. (1992) }\end{array}$ \\
\hline
\end{tabular}


Table 2.31: Allocation enablers/barriers

\begin{tabular}{|c|c|c|c|}
\hline $\begin{array}{l}\text { General } \\
\text { Factors }\end{array}$ & $\begin{array}{l}\text { Enablers/ } \\
\text { Barriers }\end{array}$ & Factors & Reference \\
\hline \multirow{3}{*}{$\begin{array}{l}\text { Presence of } \\
\text { experiences, } \\
\text { skills and } \\
\text { knowledge: } \\
\text { Factors which } \\
\text { indicate the } \\
\text { presence or } \\
\text { lack of } \\
\text { experiences, } \\
\text { skills and } \\
\text { knowledge of } \\
\text { parties }\end{array}$} & \multirow[t]{2}{*}{$\begin{array}{l}\text { Presence of } \\
\text { experiences, } \\
\text { skills and } \\
\text { knowledge }\end{array}$} & $\begin{array}{l}\text { Exchange of } \\
\text { knowledge }\end{array}$ & $\begin{array}{l}\text { Beach et al. (2005); Cao } \\
\text { and Zhang (2011); Fawcett } \\
\text { et al. (2008a, 2008b); } \\
\text { Reniers et al. (2010) }\end{array}$ \\
\hline & & $\begin{array}{l}\text { Step-by-step } \\
\text { approach: start } \\
\text { with smaller SCCs, } \\
\text { if successful } \\
\text { take next step }\end{array}$ & $\begin{array}{l}\text { Min et al. (2005); } \\
\text { Niederkofler }(1991){ }^{*}, \mathrm{RE} \\
\text { perspective }\end{array}$ \\
\hline & $\begin{array}{l}\text { Lack of } \\
\text { experiences, } \\
\text { skills and } \\
\text { knowledge }\end{array}$ & $\begin{array}{l}\text { Lack of } \\
\text { experiences on how } \\
\text { to build SCCs }\end{array}$ & Fawcett et al. (2015) \\
\hline \multirow{5}{*}{$\begin{array}{l}\text { Presence of } \\
\text { (fair) } \\
\text { benefits: } \\
\text { Factors which } \\
\text { indicate the } \\
\text { presence or } \\
\text { lack of } \\
\text { benefits and } \\
\text { their fairness }\end{array}$} & \multirow[t]{2}{*}{$\begin{array}{l}\text { Presence of } \\
\text { (fair) } \\
\text { benefits }\end{array}$} & Benefits sharing & $\begin{array}{l}\text { Anbanandam et al. (2011); } \\
\text { Fawcett et al. (2008a); } \\
\text { Gibson et al. (2002); } \\
\text { Lambert et al. (1999); } \\
\text { Matopoulos et al. (2007) }\end{array}$ \\
\hline & & $\begin{array}{l}\text { Awareness of } \\
\text { possible benefits }\end{array}$ & $\begin{array}{l}\text { Beach et al. (2005); Bucklin } \\
\text { and Sengupta (1993); Min } \\
\text { et al. (2005); }{ }^{*}, \mathrm{RE} \\
\text { perspective }\end{array}$ \\
\hline & \multirow{3}{*}{$\begin{array}{l}\text { Lack of } \\
\text { (fair) } \\
\text { benefits }\end{array}$} & $\begin{array}{l}\text { Adjustment of } \\
\text { internal process }\end{array}$ & $*$, MA perspective \\
\hline & & $\begin{array}{l}\text { Difficulty in } \\
\text { determining the } \\
\text { costs and } \\
\text { (monetary) } \\
\text { benefits }\end{array}$ & $\begin{array}{l}\text { Cruijssen et al. (2007a); } \\
\text { Fawcett and Magnan (2002) }\end{array}$ \\
\hline & & $\begin{array}{l}\text { Difficulty in } \\
\text { establishing a fair } \\
\text { allocation of the } \\
\text { benefits }\end{array}$ & Cruijssen et al. (2007a); ${ }^{*}$ \\
\hline
\end{tabular}


Table 2.32: Allocation enablers/barriers

\begin{tabular}{|c|c|c|c|}
\hline $\begin{array}{l}\text { General } \\
\text { Factors }\end{array}$ & $\begin{array}{l}\text { Enablers/ } \\
\text { Barriers }\end{array}$ & Factors & Reference \\
\hline \multirow[t]{7}{*}{$\begin{array}{l}\text { Presence of } \\
\text { (fair) } \\
\text { benefits }\end{array}$} & \multirow[t]{7}{*}{$\begin{array}{l}\text { Lack of } \\
\text { (fair) } \\
\text { benefits }\end{array}$} & $\begin{array}{l}\text { Difficulty in } \\
\text { establishing a fair } \\
\text { allocation of } \\
\text { shared workload }\end{array}$ & Cruijssen et al. (2007a) \\
\hline & & Efficiency concerns & $\begin{array}{l}*, \mathrm{LSP} \text { and MA } \\
\text { perspective }\end{array}$ \\
\hline & & $\begin{array}{l}\text { Lack of benefit } \\
\text { sharing }\end{array}$ & $\begin{array}{l}\text { Fawcett et al. (2008a); } \\
\text { Richey Jr et al. (2010) }\end{array}$ \\
\hline & & $\begin{array}{l}\text { Lack of mutual } \\
\text { benefits/ } \\
\text { profitability } \\
\text { for either party }\end{array}$ & Lambert et al. (1999) \\
\hline & & $\begin{array}{l}\text { Local nature of } \\
\text { SCC }\end{array}$ & Walker et al. (2008) \\
\hline & & $\begin{array}{l}\text { Strategic benefits } \\
\text { unclear }\end{array}$ & Akintoye et al. (2000) \\
\hline & & $\begin{array}{l}\text { Unfairness in cost } \\
\text { and pricing }\end{array}$ & Lambert et al. (1999) \\
\hline \multirow{3}{*}{$\begin{array}{l}\text { Presence of } \\
\text { flexibility: } \\
\text { Factors } \\
\text { which } \\
\text { indicate the } \\
\text { presence or } \\
\text { lack of } \\
\text { flexibility }\end{array}$} & $\begin{array}{l}\text { Presence of } \\
\text { flexibility }\end{array}$ & Flexibility & $\begin{array}{l}\text { Gibson et al. (2002); } \\
\text { Niederkofler (1991) }\end{array}$ \\
\hline & \multirow[t]{2}{*}{$\begin{array}{l}\text { Lack of } \\
\text { flexibility }\end{array}$} & $\begin{array}{l}\text { Failure to respond } \\
\text { to changes }\end{array}$ & Lambert et al. (1999) \\
\hline & & Lack of flexibility & $*$, LSP perspective \\
\hline $\begin{array}{l}\text { Presence of } \\
\text { goodwill: } \\
\text { Factors } \\
\text { which } \\
\text { indicate the } \\
\text { presence or } \\
\text { lack of value } \\
\text { of the SCC }\end{array}$ & $\begin{array}{l}\text { Presence of } \\
\text { goodwill }\end{array}$ & Goodwill & $\begin{array}{l}\text { Niederkofler (1991); *, RE } \\
\text { perspective }\end{array}$ \\
\hline
\end{tabular}


Table 2.33: Allocation enablers/barriers

\begin{tabular}{|c|c|c|c|}
\hline $\begin{array}{l}\text { General } \\
\text { Factors }\end{array}$ & $\begin{array}{l}\text { Enablers/ } \\
\text { Barriers }\end{array}$ & Factors & Reference \\
\hline $\begin{array}{l}\text { Presence of } \\
\text { interaction: } \\
\text { Factors which } \\
\text { indicate the } \\
\text { presence or } \\
\text { lack of } \\
\text { interaction }\end{array}$ & $\begin{array}{l}\text { Presence of } \\
\text { interaction }\end{array}$ & $\begin{array}{l}\text { Senior } \\
\text { management } \\
\text { interaction }\end{array}$ & $\begin{array}{l}\text { Fawcett et al. (2008a, } \\
2008 b)\end{array}$ \\
\hline \multirow{3}{*}{$\begin{array}{l}\text { Presence of } \\
\text { interdepen- } \\
\text { dence: Factors } \\
\text { which indicate } \\
\text { the presence or } \\
\text { lack of a } \\
\text { relations between } \\
\text { independent } \\
\text { parties }\end{array}$} & \multirow{2}{*}{$\begin{array}{l}\text { Presence of } \\
\text { interdepen- } \\
\text { dence }\end{array}$} & $\begin{array}{l}\text { Interdepen- } \\
\text { dence }\end{array}$ & $\begin{array}{l}\text { Richey Jr et al. (2010); } \\
\text { Ryu et al. (2009); * }\end{array}$ \\
\hline & & Mutuality & $\begin{array}{l}\text { Barratt (2004); Lambert } \\
\text { et al. (1999) }\end{array}$ \\
\hline & $\begin{array}{l}\text { Lack of } \\
\text { interdepend- } \\
\text { ence }\end{array}$ & $\begin{array}{l}\text { Lack of } \\
\text { interdepend- } \\
\text { ence }\end{array}$ & Fawcett and Magnan (2002) \\
\hline $\begin{array}{l}\text { Presence of } \\
\text { leadership: }\end{array}$ & $\begin{array}{l}\text { Presence of } \\
\text { leadership }\end{array}$ & Leadership & $\begin{array}{l}\text { Perry and Sohal (2001); } \\
\text { Riggin et al. (1992) }\end{array}$ \\
\hline $\begin{array}{l}\text { Factors which } \\
\text { indicate the } \\
\text { presence or } \\
\text { lack of } \\
\text { leadership }\end{array}$ & $\begin{array}{l}\text { Lack of } \\
\text { leadership }\end{array}$ & $\begin{array}{l}\text { Lack of } \\
\text { leadership }\end{array}$ & Fawcett et al. (2015) \\
\hline $\begin{array}{l}\text { Presence of } \\
\text { long-term } \\
\text { relationship: } \\
\text { Factors which } \\
\text { indicate the } \\
\text { presence or }\end{array}$ & $\begin{array}{l}\text { Presence of } \\
\text { long-term } \\
\text { relationship }\end{array}$ & $\begin{array}{l}\text { Long-term } \\
\text { relationship }\end{array}$ & $\begin{array}{l}\text { Akintoye et al. (2000); } \\
\text { Anbanandam et al. } \\
\text { (2011); Beach et al. } \\
\text { (2005); Ganesan (1994); } \\
\text { Gibson et al. (2002); } \\
\text { Min et al. (2005) }\end{array}$ \\
\hline $\begin{array}{l}\text { lack of a } \\
\text { long-term } \\
\text { relationship }\end{array}$ & $\begin{array}{l}\text { Lack of } \\
\text { long-term } \\
\text { relationship }\end{array}$ & $\begin{array}{l}\text { Short-term } \\
\text { relationship }\end{array}$ & $\begin{array}{l}\text { Niederkofler }(1991) ;{ }^{*}, \\
\text { MA perspective }\end{array}$ \\
\hline
\end{tabular}


Table 2.34: Allocation enablers/barriers

\begin{tabular}{|c|c|c|c|}
\hline $\begin{array}{l}\text { General } \\
\text { Factors }\end{array}$ & $\begin{array}{l}\text { Enablers/ } \\
\text { Barriers }\end{array}$ & Factors & Reference \\
\hline $\begin{array}{l}\text { Presence of } \\
\text { neglecting } \\
\text { money in the } \\
\text { first meeting: }\end{array}$ & $\begin{array}{l}\text { Presence of } \\
\text { neglecting } \\
\text { money in the } \\
\text { first meeting }\end{array}$ & $\begin{array}{l}\text { Neglecting money } \\
\text { in the first } \\
\text { meeting }\end{array}$ & $*$ \\
\hline $\begin{array}{l}\text { Factors which } \\
\text { indicate the } \\
\text { presence or } \\
\text { lack of not } \\
\text { talking about } \\
\text { money in the } \\
\text { first meeting }\end{array}$ & $\begin{array}{l}\text { Lack of } \\
\text { neglecting } \\
\text { money in the } \\
\text { first meeting }\end{array}$ & $\begin{array}{l}\text { Difficulty to speak } \\
\text { about money in the } \\
\text { first meeting }\end{array}$ & $\begin{array}{l}*, \text { MA and LSP } \\
\text { perspective }\end{array}$ \\
\hline $\begin{array}{l}\text { Presence of no } \\
\text { opportunistic } \\
\text { behavior: } \\
\text { Factors which } \\
\text { indicate the } \\
\text { presence or } \\
\text { lack of } \\
\text { opportunistic } \\
\text { behavior }\end{array}$ & $\begin{array}{l}\text { Lack of no } \\
\text { opportunistic } \\
\text { behavior }\end{array}$ & $\begin{array}{l}\text { Opportunistic } \\
\text { behavior }\end{array}$ & $\begin{array}{l}\text { Ellram and Cooper } \\
\text { (1990), shipper and LSP } \\
\text { perspective; Lambert } \\
\text { et al. (1999) }\end{array}$ \\
\hline $\begin{array}{l}\text { Presence of } \\
\text { partner } \\
\text { contribution: }\end{array}$ & $\begin{array}{l}\text { Presence of } \\
\text { partner } \\
\text { contribution }\end{array}$ & $\begin{array}{l}\text { Involvement of } \\
\text { the partners }\end{array}$ & Beach et al. (2005) \\
\hline $\begin{array}{l}\text { Factors which } \\
\text { indicate the } \\
\text { presence or } \\
\text { lack of } \\
\text { partner } \\
\text { contribution }\end{array}$ & $\begin{array}{l}\text { Lack of } \\
\text { partner } \\
\text { contribution }\end{array}$ & $\begin{array}{l}\text { Lack of } \\
\text { involvement } \\
\text { of partner }\end{array}$ & $\begin{array}{l}\text { Fawcett et al. (2008a); } \\
\text { Niederkofler (1991) }\end{array}$ \\
\hline
\end{tabular}


Table 2.35: Allocation enablers/barriers

\begin{tabular}{|c|c|c|c|}
\hline $\begin{array}{l}\text { General } \\
\text { Factors }\end{array}$ & $\begin{array}{l}\text { Enablers/ } \\
\text { Barriers }\end{array}$ & Factors & Reference \\
\hline \multirow{3}{*}{$\begin{array}{l}\text { Presence of } \\
\text { planning: } \\
\text { Factors which } \\
\text { indicate the } \\
\text { presence or } \\
\text { lack of } \\
\text { deciding how to } \\
\text { do something }\end{array}$} & $\begin{array}{l}\text { Presence of } \\
\text { planning }\end{array}$ & $\begin{array}{l}\text { Joint business } \\
\text { planning }\end{array}$ & $\begin{array}{l}\text { Akintoye et al. (2000); } \\
\text { Gibson et al. (2002); } \\
\text { Heikkilä (2002); Lambert } \\
\text { et al. (1999); Riggin et al. } \\
(1992)\end{array}$ \\
\hline & \multirow[t]{2}{*}{$\begin{array}{l}\text { Lack of } \\
\text { planning }\end{array}$} & $\begin{array}{l}\text { Internal planning } \\
\text { failure }\end{array}$ & Richey Jr et al. (2009) \\
\hline & & $\begin{array}{l}\text { Poor upfront } \\
\text { planning }\end{array}$ & Lambert et al. (1999) \\
\hline \multirow[b]{2}{*}{$\begin{array}{l}\text { Presence of } \\
\text { reliability: } \\
\text { Factors which } \\
\text { indicate the } \\
\text { presence or } \\
\text { lack of } \\
\text { reliability }\end{array}$} & \multirow{2}{*}{$\begin{array}{l}\text { Presence of } \\
\text { reliability }\end{array}$} & Reliability & Heikkilä (2002) \\
\hline & & $\begin{array}{l}\text { Reliability of } \\
\text { supply }\end{array}$ & Akintoye et al. (2000) \\
\hline \multirow{7}{*}{$\begin{array}{l}\text { Presence of } \\
\text { resources: } \\
\text { Factors which } \\
\text { indicate the } \\
\text { presence or } \\
\text { lack of } \\
\text { useful or } \\
\text { valuable } \\
\text { resources or } \\
\text { quality of } \\
\text { a partner }\end{array}$} & \multirow[t]{6}{*}{$\begin{array}{l}\text { Presence of } \\
\text { resources }\end{array}$} & $\begin{array}{l}\text { Availability and } \\
\text { quality of non- } \\
\text { financial resources }\end{array}$ & $\begin{array}{l}\text { Akintoye et al. (2000), } \\
\text { construction industry, MA } \\
\text { perspective; Riggin et al. } \\
(1992)\end{array}$ \\
\hline & & $\begin{array}{l}\text { External innovation } \\
\text { potential }\end{array}$ & Reniers et al. (2010) \\
\hline & & Financial resources & $\begin{array}{l}\text { Boddy et al. (2000); } \\
\text { Reniers et al. (2010); Riggin } \\
\text { et al. (1992) }\end{array}$ \\
\hline & & $\begin{array}{l}\text { Management of } \\
\text { resources }\end{array}$ & Riggin et al. (1992) \\
\hline & & Resources & Barratt (2004) \\
\hline & & Shared resources & Cao and Zhang (2011) \\
\hline & $\begin{array}{l}\text { Lack of } \\
\text { resources }\end{array}$ & $\begin{array}{l}\text { Lack of } \\
\text { resources }\end{array}$ & $\begin{array}{l}\text { Fawcett et al. (2012); } \\
\text { Fawcett et al. (2008a); } \\
\text { Jharkharia and Shankar } \\
\text { (2005); Niederkofler (1991); } \\
\text { Walker et al. (2008) }\end{array}$ \\
\hline
\end{tabular}


Table 2.36: Allocation enablers/barriers

\begin{tabular}{|c|c|c|c|}
\hline $\begin{array}{l}\text { General } \\
\text { Factors }\end{array}$ & $\begin{array}{l}\text { Enablers/ } \\
\text { Barriers }\end{array}$ & Factors & Reference \\
\hline \multirow{3}{*}{$\begin{array}{l}\text { Presence of } \\
\text { resources }\end{array}$} & \multirow{3}{*}{$\begin{array}{l}\text { Lack of } \\
\text { resources }\end{array}$} & Missing capacity & $*$, RE perspective \\
\hline & & Missing employee & $*$, RE perspective \\
\hline & & $\begin{array}{l}\text { Not enough time } \\
\text { and energy }\end{array}$ & $*$ \\
\hline \multirow{5}{*}{$\begin{array}{l}\text { Presence of } \\
\text { right contact } \\
\text { person: } \\
\text { Factors which } \\
\text { indicate the } \\
\text { presence or } \\
\text { lack of a } \\
\text { compatible } \\
\text { contact } \\
\text { person }\end{array}$} & \multirow{2}{*}{$\begin{array}{l}\text { Presence of } \\
\text { right contact } \\
\text { person }\end{array}$} & $\begin{array}{l}\text { Joint decision } \\
\text { making }\end{array}$ & Barratt (2004) \\
\hline & & $\begin{array}{l}\text { Relation to contact } \\
\text { person }\end{array}$ & $*$ \\
\hline & \multirow{3}{*}{$\begin{array}{l}\text { Lack of } \\
\text { right } \\
\text { contact } \\
\text { person }\end{array}$} & $\begin{array}{l}\text { Different ways of } \\
\text { thinking between } \\
\text { contact persons }\end{array}$ & $*$, MA and RE perspective \\
\hline & & $\begin{array}{l}\text { Inappropriate } \\
\text { liaison } \\
\text { managers }\end{array}$ & Niederkofler (1991) \\
\hline & & $\begin{array}{l}\text { Wrong contact } \\
\text { person }\end{array}$ & $*, \mathrm{RE}$ perspective \\
\hline \multirow[t]{2}{*}{$\begin{array}{l}\text { Presence of } \\
\text { risk sharing: } \\
\text { Factors which } \\
\text { indicate the } \\
\text { presence or } \\
\text { lack of risk } \\
\text { sharing }\end{array}$} & $\begin{array}{l}\text { Presence of } \\
\text { risk sharing }\end{array}$ & Risk sharing & $\begin{array}{l}\text { Anbanandam et al. (2011); } \\
\text { Beach et al. (2005); Fawcett } \\
\text { et al. (2008a); Gibson et al. } \\
\text { (2002); Lambert et al. } \\
\text { (1999); Matopoulos et al. } \\
(2007)\end{array}$ \\
\hline & $\begin{array}{l}\text { Lack of risk } \\
\text { sharing }\end{array}$ & $\begin{array}{l}\text { Lack of willingness } \\
\text { to share risk }\end{array}$ & $\begin{array}{l}\text { Fawcett et al. (2008a, } \\
\text { 2008b); Richey Jr et al. } \\
(2010)\end{array}$ \\
\hline \multirow{2}{*}{$\begin{array}{l}\text { Presence of } \\
\text { time: Factors } \\
\text { which indicate } \\
\text { the presence } \\
\text { or lack of } \\
\text { enough time } \\
\end{array}$} & \multirow[t]{2}{*}{$\begin{array}{l}\text { Presence of } \\
\text { time }\end{array}$} & $\begin{array}{l}\text { Factor time: launch } \\
\text { the SCC at the } \\
\text { right time }\end{array}$ & $*$, RE perspective \\
\hline & & $\begin{array}{l}\text { No time pressure at } \\
\text { the negotiation } \\
\text { process }\end{array}$ & Niederkofler (1991) \\
\hline
\end{tabular}


Table 2.37: Allocation enablers/barriers

\begin{tabular}{|c|c|c|c|}
\hline $\begin{array}{l}\text { General } \\
\text { Factors }\end{array}$ & $\begin{array}{l}\text { Enablers/ } \\
\text { Barriers }\end{array}$ & Factors & Reference \\
\hline $\begin{array}{l}\text { Presence of } \\
\text { tolerance: } \\
\text { Factors which } \\
\text { indicate the } \\
\text { presence or } \\
\text { lack of the } \\
\text { willingness to } \\
\text { accept behavior } \\
\text { and beliefs } \\
\text { which are } \\
\text { different from } \\
\text { your own }\end{array}$ & $\begin{array}{l}\text { Presence } \\
\text { of } \\
\text { tolerance }\end{array}$ & $\begin{array}{l}\text { High degree of } \\
\text { tolerance }\end{array}$ & Niederkofler (1991) \\
\hline \multirow{9}{*}{$\begin{array}{l}\text { Presence of } \\
\text { tools: Factors } \\
\text { which indicate } \\
\text { the presence } \\
\text { or lack of e.g. } \\
\text { aligned } \\
\text { methods and } \\
\text { measurements }\end{array}$} & \multirow[t]{9}{*}{$\begin{array}{l}\text { Presence } \\
\text { of } \\
\text { tools }\end{array}$} & $\begin{array}{l}\text { Adaptation of the } \\
\text { tools/ measurements } \\
\text { for the supply chain }\end{array}$ & $\begin{array}{l}\text { Barratt (2004); Fawcett et } \\
\text { al. (2008a, 2008b); Min and } \\
\text { Zhou (2002) }\end{array}$ \\
\hline & & $\begin{array}{l}\text { Conflict resolution } \\
\text { techniques/ } \\
\text { management }\end{array}$ & $\begin{array}{l}\text { Beach et al. (2005); } \\
\text { Heikkilä (2002) }\end{array}$ \\
\hline & & $\begin{array}{l}\text { Education and } \\
\text { training }\end{array}$ & $\begin{array}{l}\text { Beach et al. (2005); Fawcett } \\
\text { et al. (2008a, 2008b) }\end{array}$ \\
\hline & & $\begin{array}{l}\text { External monitoring } \\
\text { failure }\end{array}$ & Richey Jr et al. (2009) \\
\hline & & Integrated teams & Beach et al. (2005) \\
\hline & & $\begin{array}{l}\text { Joint problem } \\
\text { solving }\end{array}$ & Mohr and Spekman (1994) \\
\hline & & $\begin{array}{l}\text { More frequent } \\
\text { meetings }\end{array}$ & $\begin{array}{l}\text { Akintoye et al. (2000); } \\
\text { Perry and Sohal (2001) }\end{array}$ \\
\hline & & Partnering workshop & Beach et al. (2005) \\
\hline & & $\begin{array}{l}\text { Same measurement } \\
\text { methods (e.g. } \\
\text { performance) }\end{array}$ & $\begin{array}{l}\text { Fawcett et al. (2008a, } \\
\text { 2008b); Fawcett and } \\
\text { Magnan (2002); Gibson } \\
\text { et al. (2002); Richey Jr } \\
\text { et al. (2010); Simatupang } \\
\text { and Sridharan (2002) }\end{array}$ \\
\hline
\end{tabular}


Table 2.38: Allocation enablers/barriers

\begin{tabular}{|c|c|c|c|}
\hline $\begin{array}{l}\text { General } \\
\text { Factors }\end{array}$ & $\begin{array}{l}\text { En- } \\
\text { ablers/ } \\
\text { Barriers }\end{array}$ & Factors & Reference \\
\hline \multirow[t]{4}{*}{$\begin{array}{l}\text { Presence of } \\
\text { tools }\end{array}$} & \multirow[t]{4}{*}{$\begin{array}{l}\text { Lack of } \\
\text { tools }\end{array}$} & $\begin{array}{l}\text { Conflicts between } \\
\text { firms/functions }\end{array}$ & $\begin{array}{l}\text { Bucklin and Sengupta } \\
\text { (1993); Fawcett et al. } \\
(2008 \text { b); Matopoulos et al. } \\
(2007)\end{array}$ \\
\hline & & Lack of training & Fawcett et al. (2008b) \\
\hline & & $\begin{array}{l}\text { No adaptation of } \\
\text { the measures/ tools }\end{array}$ & $\begin{array}{l}\text { Fawcett et al. (2008a); } \\
\text { Richey Jr et al. (2010) }\end{array}$ \\
\hline & & $\begin{array}{l}\text { Non-aligned } \\
\text { measures }\end{array}$ & $\begin{array}{l}\text { Fawcett and Magnan } \\
(2002) ; \text { Fawcett et al. } \\
(2008 \mathrm{a}, 2008 \mathrm{~b}) ; \text { Fawcett } \\
\text { et al. (2015); Richey Jr } \\
\text { et al. (2010); Simatupang } \\
\text { and Sridharan (2002) }\end{array}$ \\
\hline \multirow[t]{2}{*}{$\begin{array}{l}\text { Presence of } \\
\text { trust: Factors } \\
\text { which indicate } \\
\text { the presence } \\
\text { or lack of } \\
\text { partners perceiving } \\
\text { each other as } \\
\text { credible and } \\
\text { benevolent }\end{array}$} & $\begin{array}{l}\text { Presence } \\
\text { of } \\
\text { trust }\end{array}$ & Trust & $\begin{array}{l}\text { Akintoye et al. (2000); } \\
\text { Anbanandam et al. (2011); } \\
\text { Barratt (2004); Beach et al. } \\
\text { (2005); Boddy et al. (2000); } \\
\text { Fawcett et al. (2008b); } \\
\text { Ganesan (1994); Gibson } \\
\text { et al. (2002); Heikkilä } \\
\text { (2002); Lambert et al. } \\
\text { (1999); Mohr and Spekman } \\
\text { (1994); Niederkofler (1991); } \\
\text { Perry and Sohal (2001); } \\
\text { Reniers et al. (2010); Ryu } \\
\text { et al. (2009); Whipple and } \\
\text { Frankel (2000); * }\end{array}$ \\
\hline & $\begin{array}{l}\text { Lack of } \\
\text { trust }\end{array}$ & $\begin{array}{l}\text { Difficulty in finding } \\
\text { a trusted partner/ } \\
\text { person to lead the } \\
\text { SCC }\end{array}$ & Cruijssen et al. (2007a) \\
\hline
\end{tabular}


2 A Comprehensive Framework for Holistic Evaluations of Potential Supply Chain Collaborations

Table 2.39: Allocation enablers/barriers

\begin{tabular}{|c|c|c|c|}
\hline $\begin{array}{l}\text { General } \\
\text { Factors }\end{array}$ & $\begin{array}{l}\text { Enablers/ } \\
\text { Barriers }\end{array}$ & Factors & Reference \\
\hline $\begin{array}{l}\text { Lack of } \\
\text { trust }\end{array}$ & $\begin{array}{l}\text { Lack of } \\
\text { trust }\end{array}$ & Lack of trust & $\begin{array}{l}\text { Fawcett et al. (2008b); } \\
\text { Fawcett et al. (2015); } \\
\text { Jharkharia and Shankar } \\
\text { (2005); Lambert et al. } \\
\text { (1999); Matopoulos et al. } \\
\text { (2007); *, RE perspective }\end{array}$ \\
\hline $\begin{array}{l}\text { Presence of } \\
\text { understand- } \\
\text { ing: } \\
\text { Factors }\end{array}$ & $\begin{array}{l}\text { Lack of } \\
\text { understand- } \\
\text { ing }\end{array}$ & $\begin{array}{l}\text { Lack of } \\
\text { understanding the } \\
\text { process }\end{array}$ & Fawcett and Magnan (2002 \\
\hline $\begin{array}{l}\text { which } \\
\text { indicate the }\end{array}$ & & $\begin{array}{l}\text { Poor understanding } \\
\text { of the SCC concept }\end{array}$ & Akintoye et al. (2000) \\
\hline $\begin{array}{l}\text { presence or } \\
\text { lack of parties } \\
\text { understanding } \\
\text { each other and } \\
\text { the concepts }\end{array}$ & & $\begin{array}{l}\text { Understanding of the } \\
\text { partner's resources } \\
\text { and interests }\end{array}$ & $\begin{array}{l}\text { Fawcett and Magnan } \\
(2002) ; \text { Niederkofler (1991) }\end{array}$ \\
\hline \multirow{6}{*}{$\begin{array}{l}\text { Presence of } \\
\text { willingness } \\
\text { to change: } \\
\text { Factors which } \\
\text { indicate the } \\
\text { presence or } \\
\text { lack of inertia }\end{array}$} & \multirow{6}{*}{$\begin{array}{l}\text { Presence of } \\
\text { willingness } \\
\text { to change }\end{array}$} & $\begin{array}{l}\text { Development of } \\
\text { integrated polices }\end{array}$ & $\begin{array}{l}\text { Simatupang and Sridharan } \\
(2002)\end{array}$ \\
\hline & & $\begin{array}{l}\text { External willingness } \\
\text { to change and } \\
\text { collaborate }\end{array}$ & Reniers et al. (2010) \\
\hline & & $\begin{array}{l}\text { Need for change } \\
\text { should be visible }\end{array}$ & Fawcett et al. (2008b) \\
\hline & & $\begin{array}{l}\text { Relationship } \\
\text { orientation }\end{array}$ & $\begin{array}{l}\text { Barratt (2004); Gibson } \\
\text { et al. (2002); Min et al. } \\
(2005)\end{array}$ \\
\hline & & $\begin{array}{l}\text { Willingness and drive } \\
\text { to change }\end{array}$ & $\begin{array}{l}*, \text { MA and LSP } \\
\text { perspective, vertical SCC }\end{array}$ \\
\hline & & $\begin{array}{l}\text { Willingness to work } \\
\text { together with } \\
\text { different parties }\end{array}$ & $*$, RE perspective \\
\hline
\end{tabular}


Table 2.40: Allocation enablers/barriers

\begin{tabular}{|c|c|c|c|}
\hline $\begin{array}{l}\text { General } \\
\text { Factors }\end{array}$ & $\begin{array}{l}\text { Enablers/ } \\
\text { Barriers }\end{array}$ & Factors & Reference \\
\hline \multirow[t]{4}{*}{$\begin{array}{l}\text { Presence } \\
\text { of willing- } \\
\text { ness to } \\
\text { change }\end{array}$} & \multirow[t]{4}{*}{$\begin{array}{l}\text { Lack of } \\
\text { willingness } \\
\text { to change }\end{array}$} & Inertia & $\begin{array}{l}\text { Fawcett et al. (2008b); Fawcett } \\
\text { et al. (2015); Niederkofler } \\
\text { (1991); Simatupang and } \\
\text { Sridharan (2002); Walker et al. } \\
(2008)\end{array}$ \\
\hline & & $\begin{array}{l}\text { Lack of } \\
\text { integration }\end{array}$ & $\begin{array}{l}\text { Fawcett et al. (2015); } \\
\text { Jharkharia and Shankar (2005) }\end{array}$ \\
\hline & & $\begin{array}{l}\text { Lack of } \\
\text { motivation } \\
\text { towards } \\
\text { integration }\end{array}$ & $\begin{array}{l}\text { Richey Jr et al. (2009); } \\
\text { Richey Jr et al. (2010) }\end{array}$ \\
\hline & & $\begin{array}{l}\text { Other } \\
\text { projects have } \\
\text { higher } \\
\text { priorities }\end{array}$ & $\begin{array}{l}\text { Jharkharia and Shankar }(2005) ; \text { *, } \\
\text { RE perspective }\end{array}$ \\
\hline
\end{tabular}




\section{C.4 Allocation Risks}

Table 2.41: Allocation risks

\begin{tabular}{|c|c|c|}
\hline General Factors & Factors & Reference \\
\hline \multirow{2}{*}{$\begin{array}{l}\text { Uncertainty of } \\
\text { additional costs: } \\
\text { Factors which indicate } \\
\text { an uncertainty of } \\
\text { additional future costs }\end{array}$} & $\begin{array}{l}\text { Risk of additional costs } \\
\text { additional costs }\end{array}$ & $*$ \\
\hline & $\begin{array}{l}\text { Uncertainty of the } \\
\text { costs }\end{array}$ & Fawcett and Magnan (2002) \\
\hline \multirow{3}{*}{$\begin{array}{l}\text { Uncertainty of changes } \\
\text { in key personnel: } \\
\text { Factors which indicate } \\
\text { an uncertainty of } \\
\text { changes in key } \\
\text { personnel }\end{array}$} & $\begin{array}{l}\text { Assignment of new } \\
\text { employees to } \\
\text { partnership teams }\end{array}$ & Landeros et al. (1995) \\
\hline & $\begin{array}{l}\text { Reassignment of } \\
\text { partnership leader }\end{array}$ & Landeros et al. (1995) \\
\hline & $\begin{array}{l}\text { Risk of partner } \\
\text { leaving the SCC } \\
\text { during collaboration }\end{array}$ & $*$, RE perspective \\
\hline \multirow{2}{*}{$\begin{array}{l}\text { Uncertainty of } \\
\text { changes in ownership: } \\
\text { Factors which indicate } \\
\text { an uncertainty of } \\
\text { changes in the } \\
\text { ownership }\end{array}$} & $\begin{array}{l}\text { Acquisition of one } \\
\text { partner by a third party }\end{array}$ & Landeros et al. (1995) \\
\hline & $\begin{array}{l}\text { Having unequal } \\
\text { share of financial } \\
\text { responsibility in } \\
\text { the SCC }\end{array}$ & Landeros et al. (1995) \\
\hline \multirow{3}{*}{$\begin{array}{l}\text { Uncertainty of } \\
\text { changes in the partner } \\
\text { base: Factors which } \\
\text { indicate an uncertainty } \\
\text { of changes in the partner } \\
\text { base }\end{array}$} & $\begin{array}{l}\text { Entrance of new } \\
\text { firms in the base }\end{array}$ & Landeros et al. (1995) \\
\hline & $\begin{array}{l}\text { Exiting of existing } \\
\text { firms from base }\end{array}$ & Landeros et al. (1995) \\
\hline & $\begin{array}{l}\text { Expanding for } \\
\text { technological } \\
\text { advantages in the } \\
\text { base }\end{array}$ & Landeros et al. (1995) \\
\hline
\end{tabular}


Table 2.42: Allocation risks

\begin{tabular}{|c|c|c|}
\hline General Factors & Factors & Reference \\
\hline \multirow[t]{2}{*}{$\begin{array}{l}\text { Uncertainty of } \\
\text { changes in the partner } \\
\text { base }\end{array}$} & $\begin{array}{l}\text { Increasing or } \\
\text { decreasing purchases } \\
\text { by firms in the base }\end{array}$ & Landeros et al. (1995) \\
\hline & $\begin{array}{l}\text { Increasing or } \\
\text { decreasing sales by } \\
\text { firms in the base }\end{array}$ & Landeros et al. (1995) \\
\hline \multirow{3}{*}{$\begin{array}{l}\text { Uncertainty of } \\
\text { climate related factors: } \\
\text { Factors which indicate } \\
\text { an uncertainty of } \\
\text { changes in the climate } \\
\text { and their impacts }\end{array}$} & $\begin{array}{l}\text { Environmental } \\
\text { issues }\end{array}$ & Landeros et al. (1995) \\
\hline & Natural disasters & Landeros et al. (1995) \\
\hline & Weather changes & Landeros et al. (1995) \\
\hline $\begin{array}{l}\text { Uncertainty of } \\
\text { competition: Factors } \\
\text { which indicate an } \\
\text { uncertainty of future } \\
\text { competition and } \\
\text { competitiveness of the } \\
\text { partner }\end{array}$ & $\begin{array}{l}\text { Entrance of new } \\
\text { competition or } \\
\text { new competitive } \\
\text { advantages in the } \\
\text { industry }\end{array}$ & Landeros et al. (1995) \\
\hline \multirow{12}{*}{$\begin{array}{l}\text { Uncertainty of } \\
\text { economy: Factors } \\
\text { which indicate an } \\
\text { uncertainty of changes } \\
\text { of the system and/or } \\
\text { industry }\end{array}$} & $\begin{array}{l}\text { Balance of } \\
\text { payment }\end{array}$ & Landeros et al. (1995) \\
\hline & Deficits & Landeros et al. (1995) \\
\hline & Depression & Landeros et al. (1995) \\
\hline & Fiscal policies & Landeros et al. (1995) \\
\hline & $\begin{array}{l}\text { Inflationary or } \\
\text { deflationary trend }\end{array}$ & Landeros et al. (1995) \\
\hline & Interest rates & Landeros et al. (1995) \\
\hline & Monetary policies & Landeros et al. (1995) \\
\hline & $\begin{array}{l}\text { Prosperous } \\
\text { business cycle }\end{array}$ & Landeros et al. (1995) \\
\hline & Recession & Landeros et al. (1995) \\
\hline & Recovery & Landeros et al. (1995) \\
\hline & Surpluses & Landeros et al. (1995) \\
\hline & Tax rates & Landeros et al. (1995) \\
\hline
\end{tabular}


Table 2.43: Allocation risks

\begin{tabular}{|c|c|c|}
\hline General Factors & Factors & Reference \\
\hline \multirow{5}{*}{$\begin{array}{l}\text { Uncertainty of } \\
\text { government: Factors } \\
\text { which indicate an } \\
\text { uncertainty of the } \\
\text { government }\end{array}$} & $\begin{array}{l}\text { Americans with } \\
\text { Disables Act }\end{array}$ & Landeros et al. (1995) \\
\hline & $\begin{array}{l}\text { Domestic content } \\
\text { requirements }\end{array}$ & Landeros et al. (1995) \\
\hline & $\begin{array}{l}\text { Equal employment } \\
\text { opportunity }\end{array}$ & Landeros et al. (1995) \\
\hline & $\begin{array}{l}\text { Safety and health } \\
\text { regulations }\end{array}$ & Landeros et al. (1995) \\
\hline & $\begin{array}{l}\text { Wage and price } \\
\text { control }\end{array}$ & Landeros et al. (1995) \\
\hline \multirow{2}{*}{$\begin{array}{l}\text { Uncertainty of high } \\
\text { dependency: Factors } \\
\text { which indicate an } \\
\text { uncertainty of the } \\
\text { dependency on another } \\
\text { partner }\end{array}$} & $\begin{array}{l}\text { Contract uncertainty; } \\
\text { parties are } \\
\text { mutually dependent }\end{array}$ & *, MA and RE perspective \\
\hline & $\begin{array}{l}\text { Risk of } \\
\text { dependency }\end{array}$ & $\begin{array}{l}\text { Ellram and Cooper }(1990), \\
\text { shipper perspective; Ganesan } \\
(1994) \text {; Niederkofler }(1991) ;{ }^{*}\end{array}$ \\
\hline $\begin{array}{l}\text { Uncertainty of losing } \\
\text { clients: Factors which } \\
\text { indicate an uncertainty } \\
\text { of losing customers }\end{array}$ & $\begin{array}{l}\text { Risk of losing } \\
\text { clients to competitors }\end{array}$ & Cruijssen et al. (2007a) \\
\hline \multirow{3}{*}{$\begin{array}{l}\text { Uncertainty of losing } \\
\text { transparency: Factors } \\
\text { which indicate an } \\
\text { uncertainty of changes } \\
\text { in transparency in the } \\
\text { future }\end{array}$} & $\begin{array}{l}\text { Risk of confidential } \\
\text { information security }\end{array}$ & $\begin{array}{l}\text { Jharkharia and Shankar } \\
(2005) ; * \text { LSP perspective }\end{array}$ \\
\hline & $\begin{array}{l}\text { Risk of information } \\
\text { transfer }\end{array}$ & $\begin{array}{l}\text { Ellram and Cooper (1990), } \\
\text { shipper perspective }\end{array}$ \\
\hline & $\begin{array}{l}\text { Uncertainty of } \\
\text { losing transparency }\end{array}$ & $*$, MA perspective \\
\hline
\end{tabular}


Table 2.44: Allocation risks

\begin{tabular}{|c|c|c|}
\hline General Factors & Factors & Reference \\
\hline \multirow{5}{*}{$\begin{array}{l}\text { Uncertainty of } \\
\text { organizational } \\
\text { socialization: Factors } \\
\text { which indicate an } \\
\text { uncertainty of changes } \\
\text { in the process through } \\
\text { which new employees } \\
\text { learn to adapt to the } \\
\text { organizational culture }\end{array}$} & $\begin{array}{l}\text { Enriching team } \\
\text { potency }\end{array}$ & Landeros et al. (1995) \\
\hline & $\begin{array}{l}\text { Establishing the } \\
\text { partnership team's } \\
\text { importance, purpose } \\
\text { and identity }\end{array}$ & Landeros et al. (1995) \\
\hline & $\begin{array}{l}\text { Setting up } \\
\text { SCC among } \\
\text { members }\end{array}$ & Landeros et al. (1995) \\
\hline & $\begin{array}{l}\text { Setting team } \\
\text { tasks }\end{array}$ & Landeros et al. (1995) \\
\hline & $\begin{array}{l}\text { Training existing } \\
\text { and new members }\end{array}$ & Landeros et al. (1995) \\
\hline \multirow{5}{*}{$\begin{array}{l}\text { Uncertainty of } \\
\text { performance } \\
\text { problems: Factors } \\
\text { which indicate an } \\
\text { uncertainty of problems } \\
\text { with the performance } \\
\text { and quality in the future }\end{array}$} & Data reliability & $*$, RE perspective \\
\hline & $\begin{array}{l}\text { Late or missed } \\
\text { deliveries }\end{array}$ & Landeros et al. (1995) \\
\hline & $\begin{array}{l}\text { Quality performance } \\
\text { problems }\end{array}$ & $\begin{array}{l}\text { Ellram and Cooper (1990), } \\
\text { shipper perspective; Landeros } \\
\text { et al. (1995); *, RE perspective }\end{array}$ \\
\hline & $\begin{array}{l}\text { Uncertainty of } \\
\text { outcome }\end{array}$ & $\begin{array}{l}\text { Jharkharia and Shankar } \\
(2005) ;^{*}, \text { RE perspective }\end{array}$ \\
\hline & $\begin{array}{l}\text { Withholding or } \\
\text { delaying necessary } \\
\text { information }\end{array}$ & Landeros et al. (1995) \\
\hline $\begin{array}{l}\text { Uncertainty of social } \\
\text { factors: Factors which } \\
\text { indicate an uncertainty } \\
\text { of social factors in the } \\
\text { future }\end{array}$ & $\begin{array}{l}\text { Personal values } \\
\text { and attitude shift }\end{array}$ & Landeros et al. (1995) \\
\hline
\end{tabular}


Table 2.45: Allocation risks

\begin{tabular}{|c|c|c|}
\hline General Factors & Factors & Reference \\
\hline \multirow{5}{*}{$\begin{array}{l}\text { Uncertainty of } \\
\text { strategic intent: } \\
\text { Factors which indicate } \\
\text { an uncertainty of a } \\
\text { change in the strategic } \\
\text { intent in the future }\end{array}$} & $\begin{array}{l}\text { Acquiring new } \\
\text { technologies to } \\
\text { exploit future } \\
\text { opportunities }\end{array}$ & Landeros et al. (1995) \\
\hline & $\begin{array}{l}\text { Capturing existing } \\
\text { markets }\end{array}$ & Landeros et al. (1995) \\
\hline & $\begin{array}{l}\text { Expanding into } \\
\text { global markets }\end{array}$ & Landeros et al. (1995) \\
\hline & $\begin{array}{l}\text { Exploiting } \\
\text { competitors' } \\
\text { weaknesses } \\
\end{array}$ & Landeros et al. (1995) \\
\hline & $\begin{array}{l}\text { Opening new } \\
\text { markets }\end{array}$ & Landeros et al. (1995) \\
\hline \multirow{5}{*}{$\begin{array}{l}\text { Uncertainty of } \\
\text { strategic match: } \\
\text { Factors which indicate } \\
\text { an uncertainty of } \\
\text { changes in the strategic } \\
\text { match in the future }\end{array}$} & $\begin{array}{l}\text { Embracing } \\
\text { just-in-time } \\
\text { philosophies }\end{array}$ & Landeros et al. (1995) \\
\hline & $\begin{array}{l}\text { Implementing total } \\
\text { quality management } \\
\text { concepts }\end{array}$ & Landeros et al. (1995) \\
\hline & $\begin{array}{l}\text { Installing cycle } \\
\text { time reducing } \\
\text { goals }\end{array}$ & Landeros et al. (1995) \\
\hline & $\begin{array}{l}\text { Partners' interest } \\
\text { and resources } \\
\text { requirements shift } \\
\text { over time }\end{array}$ & Niederkofler (1991) \\
\hline & $\begin{array}{l}\text { Requiring } \\
\text { continuous } \\
\text { improvement } \\
\text { objectives }\end{array}$ & Landeros et al. (1995) \\
\hline $\begin{array}{l}\text { Uncertainty of } \\
\text { technology: Factors } \\
\text { which indicate an } \\
\text { uncertainty of } \\
\text { technology in the future }\end{array}$ & $\begin{array}{l}\text { New product or } \\
\text { process advancements }\end{array}$ & Landeros et al. (1995) \\
\hline
\end{tabular}




\section{D Extract of the Interview Guide}

Date:

Location:

Organization:

Party: LSP Manufacturer Retailer

Name of the interviewer(s):

Name/Position/Function of the Interviewee:

Other participant during interview:

Name/Position/Function:

Record of the interview: Audio Video

1. Introducing questions

(a) General questions about the company (volume, size, turnover, number of employees etc.).

(b) What does "supply chain collaboration" in general mean for you and your company?

(c) What comes into your mind when you hear the term "supply chain collaboration"? (horizontal/vertical/lateral)

2. Discussion about collaborations

(a) Short description of the collaboration; which supply chain parties were involved in this supply chain collaboration? Names? Number of participants in total? Number of participants per party?

(b) What type of collaborations? (e.g. horizontal, vertical or lateral SCC)

(c) When did you start to collaborate?

(d) Was the supply chain collaboration successful?

3. Discussion about enablers for launching a collaboration

(Definition of the category enabler)

(a) Are there any prerequisites (enablers) that enable you to launch the collaboration? Which? How would you define them? Why? How important? 
2 A Comprehensive Framework for Holistic Evaluations of Potential Supply Chain Collaborations

4. Discussion about forces/benefits for launching a collaboration (Definition of the categories force and benefit)

(a) What made you launch the specific SCCs? Why are you willing to collaborate with your fellow supply chain parties? Which factors? Why? How important?

5. Discussion about barriers/risks of launching a collaboration (Definition of the category barrier and risk)

(a) What are barriers that could limit the success of the collaboration before launching the SCC? Which? Why? How important?

(b) What are risks that might occur during the collaboration? Which? Why? How important? 


\section{Chapter 3}

\section{Disagreement on the Gain Sharing Method in Supply Chain Collaborations ${ }^{1}$}

\subsection{Introduction}

The case studies in Section 2.4 confirmed the importance of cost reduction as a motivation to launch collaborations. This motivation factor is at the same time also one of the main challenges for collaborations. By reducing costs through SCC, a coalition gain can be achieved, which also needs to be distributed among the collaborative parties. This distribution is, according to Cruijssen et al. (2007a) and Leng and Parlar (2009), a main barrier for the implementation and the success of an SCC. The case studies in the Dutch FMCG industry described in Chapter 2 stress the findings of Cruijssen et al. (2007a) and Leng and Parlar (2009), as well. If one partner is not satisfied with its allocated share or feels like not receiving a fair portion of the coalition gain, future SCCs are less likely to occur (Jap, 2001). Cruijssen et al. (2007b) also mention that mistrust regarding the fairness of the applied allocation method already caused many SCC failures. This is stressed by Cruijssen (2012), who state that having a fair allocation method, one that is also perceived as fair by the collaborative parties, is essential for a successful and sustainable SCC. Based on the importance of fair gain sharing, several researchers

\footnotetext{
${ }^{1}$ This chapter is based on the paper Jung et al. (2018a)
} 
already developed gain sharing methods to allocate the coalition gain among the collaborative parties (e.g. Frisk et al., 2010; Liu et al., 2010; Schmeidler, 1969; Shapley, 1953; Tijs and Driessen, 1986). The general idea of these gain sharing methods is to distribute the gains in such a way that everyone is satisfied to ensure the establishment and sustainability as well as to realize the potential of the SCC (Liu et al., 2010). Although the above-mentioned statements express the importance of the acceptance of a gain sharing method by all parties, until now the actual acceptance levels of gain sharing methods have not been investigated. This chapter attempts to fill this gap. It extends the works by Cruijssen et al. (2007a), Leng and Parlar (2009) as well as the work presented in Chapter 2 by investigating the parties' acceptance levels of selected gain sharing methods. Section 3.3.1, provides an overview of the selected gain sharing methods. In this chapter the allocation methods are outlined as cost allocation methods since this is common in current literature. Dividing the costs or gains among the coalition partners is equivalent, since the total gains of all collaborative parties equals the difference between the sum of all stand-alone costs and the total collaborative costs. In this study, the acceptance of the selected gain sharing methods is investigated in vertical three-echelon SCCs (manufacturer, LSP, retailer) in the Dutch FMCG industry. The FMCG industry was chosen as SCCs are very important for all parties in this industry in order to be competitive (de Kok et al., 2015). As a result, to ensure sustainable SCCs in the FMCG industry all collaborative parties have to be satisfied with and accept the assigned gain share.

In order to investigate the cause for the acceptance or rejection of a gain sharing method, the influence of behavioral decision-making aspects on the acceptance levels of these gain sharing methods is examined. Special attention is paid to two behavioral decision-making aspects: information availability and cognitive biases. The focus is first on information availability, since in the case studies a connection between the access of information and gain sharing was identified, see Section 2.4.3. Second, cognitive biases are considered due to their close connection to information availability. Parties rely on cognitive biases if incomplete information is provided (Sterman, 1989). In addition to the investigation of the acceptance levels of gain sharing methods, the incorporation of behavioral research literature within SCM research is another contribution of this chapter. For a long time, the predominant assumption in economics was that human beings are rational thinking agents, which implies that decisions are made in a rational and consistent way (Sterman, 
1989). However, human beings are limited due to restrictions in available time, information and cognitive capabilities (Simon, 1979). They tend to rely on heuristics or cognitive biases to deal with complex problems (Schenk, 2011). Until now, the research published in the fields of logistics and SCM literature dealing with the influence of human behavior, judgment and decision-making is limited. However, to ensure practical validity it is necessary to incorporate behavioral research in SCM studies (Tokar, 2010). This is stressed by Mantel et al. (2006), who outline the greater understanding of decisions made in SCM by integrating decision-making literature in SCM research.

The remainder of this chapter is structured as follows. In Section 3.2, gain sharing methods are outlined and the behavioral decision-making literature is discussed. Next, the acceptance of selected gain sharing methods as well as the influence of behavioral aspects on the acceptance levels of these allocation methods are investigated. The research methodology is outlined in Section 3.3. In Section 3.4, the statistical analysis and results are presented, followed by a discussion in Section 3.5. The chapter concludes with a short summary, implications for theory and practice as well as directions for further research.

\section{$3.2 \quad$ Literature Review}

\subsubsection{Gain Sharing Methods}

As the goal of an SCC is to increase the participants' efficiency and since collaboration often results in additional gains, a great deal of scientific literature focuses on the identification of efficient gain sharing methods (e.g Frisk et al., 2010; Liu et al., 2010; Tijs and Driessen, 1986). Verdonck et al. (2016) provide a structured review of gain sharing methods applied in logistics collaborations distinguishing between proportional sharing mechanisms, allocation mechanisms using game theory concepts and allocation techniques designed to cope with additional cooperation properties.

In practice, the most commonly used gain sharing method is the proportional allocation method (Liu et al., 2010). In this case, coalition gains are shared among the parties in proportion to their individual cost level or the volume they have to transport within the context of the collaboration (Verdonck et al., 2016). These 
methods are preferred in practice due to their simplicity and transparency (Cruijssen et al., 2007b).

Furthermore, an SCC matches the structure of a cooperative game. The collaboration process results in an allocation of gains to each collaborative partner that may be considered equivalent to the outcome of a cooperative game. A relevant concept in this context is the core (Shapley, 1952). The core of a game consists of all gain allocations that are budget balanced and guarantee that no single partner or coalition of partners benefits from leaving the collaboration. Another well-known gain sharing method based on the foundations of game theory is the Shapley value (Shapley, 1953). This value allocates to each partner the weighted average of its contributions to all (sub)coalitions, assuming that the grand coalition is formed successively, with one party joining at a time. A more complex game theoretic sharing mechanism is the Nucleolus. This method minimizes the maximal excess, which constitutes the difference between the total costs of a coalition and the sum of the costs allocated to its parties (Schmeidler, 1969).

Finally, several authors have developed distinct gain sharing mechanisms that account for specific collaboration characteristics (Verdonck et al., 2016). Tijs and Driessen (1986) discuss allocation methods that split the collaborative costs in a separable and a non-separable part. The separable part is directly linked and assigned to a specific party. The remaining costs, the non-separable part, have to be divided among the parties. Tijs and Driessen (1986) mention different ways of how the non-separable part of the costs can be allocated. They discuss the equal charge method (ECM), the alternative cost avoided method (ACAM) and the separable cost remaining benefits (SCRB). They also introduce a new method, the cost gap method (CGM). Frisk et al. (2010) use their equal profit method (EPM) to identify stable allocations that minimize the largest relative difference in cost savings between any pair of collaborative parties. The weighted relative savings model (WRSM) proposed by Liu et al. (2010) is similar to the EPM, but takes additionally the difference in the parties' contribution into account. The gain sharing methods developed by Özener and Ergun (2008) ensure that existing parties do not lose savings when an additional party joins the collaboration.

Although simple proportional methods are appealing to collaborative parties in practice due to their simplicity and transparency, Cruijssen et al. (2007b) point out that these simple methods might systematically undervalue a party's true share in the SCC success. In the long run, this might lead to frustration and one party 
leaving the collaboration. Unlike these simple methods, game theoretic allocation methods "objectively take into account each player's impact and produce compromise allocations that distribute the benefits of cooperation based on clear fairness properties" (Cruijssen et al., 2007a). Therefore, in the case study introduced in Section 3.3 the focus is on game theoretic allocation methods as well as allocation techniques designed to cope with additional cooperation properties.

\subsubsection{Behavioral Decision-Making}

For a long time, the predominant assumption in economics was that human beings think rational. However, Simon (1979) describes a restriction on rational decisionmaking due to limitations of available time, information and cognitive capabilities of the decision-makers. Although people think that they are rationally thinking creatures, their thinking, their memory and also their decision-making are influenced by cognitive biases. To date, a wide range of cognitive biases have been identified. Among these is the recency bias, according to which people tend to put more weight on the latest information they receive (Hallowell and Gambatese, 2009). Another example is the so-called salience bias. Human beings influenced by this bias tend to focus on the most easily-recognizable items or information of a concept and ignore the ones that are not as apparent (Schenk, 2011). The choicesupportive bias states that people tend to feel positive about their choice, even if the choice has a flaw (Mather and Johnson, 2000). As a final example, the framing effect bias could be named. According to De Martino et al. (2006), human beings are remarkably vulnerable to the manner in which options are presented, which is the so-called framing effect. Therefore, when facing decision problems with identical consequences, people's decisions may differ depending on how the options are presented; in a positive, in terms of gains, or in a negative, in terms of losses, frame.

As already outlined in the introduction, the focus of this chapter is on two behavioral aspects: information availability and cognitive biases. Attention is paid to these two aspects due to the identified connection between the availability of information with gain sharing as well as with cognitive biases, see Sterman (1989) as well as Chapter 2. The availability of time might also have an influence on the acceptance of a gain sharing method. However, in the case studies introduced in Chapter 2 no connection between these two aspects was identified for the Dutch FMCG industry. Nevertheless, time availability might be an interesting aspect to take into account in further research. 


\subsection{Research Procedure}

To the best of the author's knowledge, no researcher has investigated the acceptance levels of gain sharing methods in practice. Therefore, this research is exploratory. According to (Yin, 2013), a case study approach is, under these conditions, the best-suited approach. Therefore, a quantitative case study approach is used. By means of the case study, the following questions will be examined:

1. What are the acceptance levels of gain sharing methods in the Dutch FMCG industry?

(a) What are the manufacturers' acceptance levels of gain sharing methods?

(b) What are the LSPs' acceptance levels of gain sharing methods?

(c) What are the retailers' acceptance levels of gain sharing methods?

2. What is the influence of behavioral decision-making aspects on the acceptance levels of gain sharing methods in the Dutch FMCG industry?

(a) What is the influence of information availability on the acceptance levels of gain sharing methods?

(b) What is the influence of cognitive biases on the acceptance levels of gain sharing methods?

The research procedure is divided into four steps. In Table 3.1, the main aspects for each step are outlined. 
Table 3.1: Research Procedure

\begin{tabular}{|c|c|c|c|}
\hline Steps & Procedure & Comments & $\begin{array}{l}\text { Refer- } \\
\text { ence }\end{array}$ \\
\hline $\begin{array}{c}\text { Step } \\
1\end{array}$ & $\begin{array}{l}\text { Variable } \\
\text { Selection }\end{array}$ & $\begin{array}{l}\text { Independent Variables } \\
\text { Gain sharing method (Nucleolus, Shapley } \\
\text { value, WCM - Power/Initiator, ECM) } \\
\text { Information availability (Phase } 1,2 \text { and 3) } \\
\text { Perspective (Manufacturer,LSP, Retailer) } \\
\text { Dependent Variable } \\
\text { Acceptance }\end{array}$ & $\begin{array}{l}\text { Section } \\
3.3 .1\end{array}$ \\
\hline $\begin{array}{c}\text { Step } \\
2\end{array}$ & $\begin{array}{l}\text { Data } \\
\text { Collection }\end{array}$ & $\begin{array}{l}\text { Online Survey } \\
\text { Accept or reject certain gain shares } \\
\text { for all allocation methods in all phases } \\
\text { Exclusively gains (ordered from the lowest to the } \\
\text { highest) are presented }\end{array}$ & $\begin{array}{l}\text { Section } \\
3.3 .2\end{array}$ \\
\hline $\begin{array}{c}\text { Step } \\
3\end{array}$ & $\begin{array}{l}\text { Population } \\
\text { and Sample } \\
\text { Selection }\end{array}$ & $\begin{array}{l}\frac{\text { Population }}{\text { Companies from the Dutch FMCG }} \\
\text { industry/participants in logistics competition } \\
\frac{\text { Sample Size }}{4 \text { manufacturers, } 4 \text { LSPs, } 4 \text { retailers }}\end{array}$ & $\begin{array}{l}\text { Section } \\
3.3 .3\end{array}$ \\
\hline $\begin{array}{c}\text { Step } \\
4\end{array}$ & $\begin{array}{l}\text { Data } \\
\text { Analysis }\end{array}$ & $\begin{array}{l}\text { Analytical Tool } \\
\text { Binary logistic regression } \\
\text { Independent Variables } \\
\text { Gain sharing method (Method): } \\
\text { Categorical variable (Baseline: Nucleolus) } \\
\text { Information availability (Phase): } \\
\text { Categorical variable (Baseline: Phase 1) } \\
\text { Perspective (Type): } \\
\text { Categorical variable (Baseline: Manufacturer) } \\
\text { Dependent Variable } \\
\text { Acceptance: Binary variable }\end{array}$ & $\begin{array}{l}\text { Section } \\
3.3 .4\end{array}$ \\
\hline
\end{tabular}




\subsubsection{Variable Selection}

In order to answer the introduced questions, the influence of three aspects - gain sharing method, information availability and perspective - on the acceptance of selected gain sharing methods is investigated. These aspects are outlined in detail in the following sections.

\section{Gain Sharing Method}

First, the influence of the gain sharing method, which represents the first variable, is investigated. The gain sharing method determines the gain that is assigned to each party. As the assigned gain shares differ among the gain sharing methods, the level of acceptance of the allocation methods are most likely different, which might uncover possible cognitive biases. In this research, the focus is on four gain sharing methods: the Shapley value, the Nucleolus and two methods based on separable and non-separable costs, the weighted charge method (WCM) and the ECM. For the WCM, two weights were chosen. Therefore, five gain sharing methods were investigated in total. The first two allocation methods are well-known game theoretic allocation methods and the most preferred methods in theory (Moulin, 1991). The other allocation methods are, according to a preliminary study, most similar to what is already used in practice. In this preliminary study, 20 companies from the Dutch FMCG industry were interviewed in order to identify their understanding of a (fair) gain sharing, their willingness to share gains and their experiences with gain sharing. The interview guide as well as some additional information are outlined in Appendix 3A.

\section{Shapley Value}

For the Shapley value, the formation of the grand coalition N, which includes every collaborative party, can be seen as a sequential process, in which the collaborative parties enter one by one. For every partner $i$, the value is defined as the average marginal contribution of the partner to every possible subcoalition $S$ of the grand coalition $N$ containing this partner. The Shapley value is based on the four axioms formulated by Shapley (1953) and can be computed by:

$$
x_{i}=\sum_{(S \cup N \backslash i)} \frac{(|S| !(|N|-|S|-1) !}{|N| !} *(c(S \cup i)-c(S))
$$

where $x_{i}$ represents the allocated costs for partner $i$. Furthermore, let $|N|$ and $|S|$ 
denote the number of partners in a grand coalition and subcoalition, respectively. Lastly, $c(S)$ represents the costs of a subcoalition $S$.

\section{Nucleolus}

The Nucleolus (Schmeidler, 1969) is based on the idea of minimizing the maximum excess. The excess is the gain the parties in a subcoalition $S$ obtain if they exit the grand coalition $N$. For a subcoalition $S$ given an allocation $x$, the excess for a subcoalition $e(x, S)$ is denoted as:

$$
e(x, S)=c(S)-\sum_{(i \in S)} x_{i}
$$

where $c(S)$ represents the costs of a subcoalition $S$.

\section{Weighted Charge Method}

The WCM is based on the idea of Tijs and Driessen (1986) that the costs are at first split in a separable $\left(m_{i}=c(N)-c(N \backslash i)\right)$ and a non-separable part $\left(c(N)-\sum_{i=0}^{N} m_{i}\right)$. The non-separable part is divided among the parties according to a specific weight $w_{i}$. The allocation for a partner $i$ is then computed as follows:

$$
x_{i}=m_{i}+\left(c(N)-\sum_{i=0}^{N} m_{i}\right) * w_{i}
$$

where $x_{i}$ represents the allocated costs for partner $i, m_{i}$ represents the separable part of the costs for partner $i$ and $c(N)$ represents the costs of the grand coalition $N$.

Based on the preliminary study (see Appendix 3A), two different types of weights could be identified, one based on the power position and one based on the initiator. In the Dutch FMCG industry, the retailer is the most powerful party. Therefore, the highest weight $w_{r}=0.5$ is assigned to the retailer. In comparison to the manufacturer, the LSP is more powerful. Therefore a weight of $w_{l}=0.3$ is allocated to the LSP and the rest $w_{m}=0.2$ is assigned to the manufacturer. Furthermore, in the Dutch FMCG industry the LSP often initiates the launch of an SCC. As a result, the highest weight $\left(w_{l}=0.4\right)$ is allocated to the LSP. The rest is equally split among the manufacturer and retailer $\left(w_{r}=w_{m}=0.3\right)$. 


\section{Equal Charge Method}

The ECM is also based on the idea of Tijs and Driessen (1986). Unlike the WCM, the non-separable part is equally distributed among the parties. Therefore, the total amount allocated to each partner $i$ is:

$$
x_{i}=m_{i}+\frac{\left(c(N)-\sum_{i=0}^{N} m_{i}\right)}{(|N|)}
$$

\section{Information Availability}

Second, the influence of the information availability, which represents the second variable, is examined. This behavioral decision-making aspect refers to the limitation of available information outlined by Simon (1979) in the context of bounded rationality. Human beings make their decisions based on cognitive biases when available information is limited (Sterman, 1989). In order to investigate the influence of information availability, three different phases were developed. The amount of information increases with each phase. In the first phase, participants only receive information about their own financial consequences. This includes information about their benefits, costs related to the SCC and the resulting profit, which is equal to the contribution they make to the coalition gain, see Table 3.2. In addition, they receive information about the gain they will receive according to each of the five gain sharing methods, see Table 3.3. In the second phase, the participants also receive information about the financial consequences of their coalition partners. Finally, in the last phase, market information for each collaborative party is included. Here, information about the market share, products and the importance of a collaboration with the other partners is included, see Figure 3.1.

Table 3.2: Information about the financial consequences after a five year SCC

\begin{tabular}{c|ccc}
\hline & Manufacturer & LSP & Retailer \\
\hline \hline Benefits & $80,000 €$ & $50,000 €$ & $250,000 €$ \\
Costs & $85,000 €$ & $10,000 €$ & $80,000 €$ \\
Profits & $-5,000 €$ & $40,000 €$ & $170,000 €$ \\
\hline
\end{tabular}


Table 3.3: Gain assigned to the different parties according to the gain sharing methods

\begin{tabular}{c|ccc}
\hline & Manufacturer & LSP & Retailer \\
\hline \hline Nucleolus & $4,333.33 €$ & $55,333.33 €$ & $145,333.33 €$ \\
Shapley & $36,333.33 €$ & $61,833.33 €$ & $106,833.33 €$ \\
WCM-Power & $41,000.00 €$ & $61,500.00 €$ & $102,500.00 €$ \\
WCM-Initiator & $61,500.00 €$ & $82,000.00 €$ & $61,500.00 €$ \\
ECM & $68,333.33 €$ & $68,333.33 €$ & $68,333.33 €$ \\
\hline
\end{tabular}

\section{Market Information}

The retailer is the biggest player on the market with a market share of $25 \%$ and is a member of a union called "Super Unie". Relationships with this party are important.

The LSP is a medium player on the market with a market share of $10 \%$.

The manufacturer is based locally and the goods are relatively easy to substitute. The manufacturer is no member of any association or union of parties from the same position in the supply chain.

Figure 3.1: Market information for each collaborative party

\section{Perspective}

Third, the influence of the perspective, which represents the third variable, is investigated. The case study focuses on a vertical SCC between one manufacturer, one LSP and one retailer. Different collaborative parties have different and therefore incomplete information. As a result, the collaborative parties will most likely show various cognitive biases since, as stated by Sterman (1989), human beings rely on cognitive biases if incomplete information are available.

\subsubsection{Data Collection}

The data of the quantitative case study was collected using online surveys. The strong methodology control is the main reason to use an online survey. In an online survey, the order of the questions, the completeness of the answers and the filtering can be controlled by the researcher (Evans and Mathur, 2005). Participants were asked in each phase of information availability and for each gain sharing method to evaluate whether they would accept or reject the assigned gain. Thereby, the order of the questions from Phase 1 to Phase 3 is crucial in order to observe the 
influence of the available information. The participants only see the outcome of the gain sharing method, but they do not know which method was applied. As a result, the acceptance of the allocation method was examined through the acceptance of a specific gain. The assigned gain shares are ranked from lowest to highest in order to prevent parties from rejecting a gain share which is lower than a previous one. Another important advantage of the online survey is that participants cannot look ahead like in a mail survey. However, not knowing the amount of questions might discourage a respondent from continuing the online survey (Evans and Mathur, 2005). In order to prevent this, a graphical progress indicator was used. The predetermined order and preventing participants from skipping ahead to later questions reduce the survey bias. Moreover, through the use of an online survey rather than a personal survey or a telephone survey, the so-called interview bias is avoided, which can always occur in case of personal contact between interviewer and respondent (Evans and Mathur, 2005). Among others, the goal of the online survey is to observe cognitive biases. Therefore, preventing biases resulting from the surveys themselves is essential. Furthermore, split samples were used. The online surveys were different for each collaborative party. According to Evans and Mathur (2005), "online surveys are particularly effective when multiple samples are involved".

The online surveys were distributed through a link to the survey URLs in an e-mail. Reminders were sent out to achieve a higher response rate. An example of the online survey can be found in Appendix 3B.

\subsubsection{Population and Sample Size Selection}

The online surveys were conducted with companies from the Dutch FMCG industry. It was selected due to the importance of SCCs for this industry (de Kok et al., 2015). In the FMCG industry, it is necessary for parties to collaborate with their supply chain partners. To ensure sustainable SCCs, all parties need to be satisfied with and accept their assigned gain share. Participants were selected from a population of 26 companies that participated in the logistics competition introduced in Chapter 2. The sample size is 12, including four manufacturers, four LSPs and four retailers. The online surveys were conducted with supply chain or logistics managers of the companies due to their experiences and expertise in SCC. 


\subsubsection{Data Analysis}

To analyze the outlined research questions, a binary logistic regression is performed (Hosmer Jr et al., 2013). In order to use the logistic regression, some data preparations have to be made. The dependent variable is a binary variable with a value equal to 1 if the gain is accepted and 0 otherwise. The three independent variables are all categorical variables. The gain sharing method variable is coded as Nucleolus, Shapley, WCM-Power, WCM-Initiator and ECM. Taking the Nucleolus as a baseline, the gain sharing variable is represented by four binaries. To represent the five gain sharing methods, only four design variables are necessary due to an intercept used in the model (Hosmer Jr et al., 2013). Information availability is represented by two variables with Phase 1 designated as the reference phase. The perspective is also represented by two variables, while the Manufacturer is taken as the baseline.

Problems with the logistic regression occurred as a consequence of a data pattern known as quasi-complete separation. Quasi-complete separation occurs if the dependent variable of an independent dummy variable is always either equal to 1 or to 0 . As a consequence, the maximum likelihood estimate does not exist. This problem often occurs if a small sample size is used (Allison, 2008). Therefore, a binary logistic regression with a penalized maximum likelihood estimation is used. The penalized maximum likelihood estimation method was proposed by Firth (1993) to reduce the bias in maximum likelihood estimates. Heinze and Schemper (2002) show that this method provides a solution for the quasi-complete separation problem. The basic idea of the penalized maximum likelihood estimation method is to introduce a modified score function which removes the bias of the maximum likelihood estimates of the coefficients (Firth, 1993). For a more elaborate explanation of this method, the reader is referred to Firth (1993) and Heinze and Schemper (2002).

Both binary logistic regressions are performed on $R$ (version 3.3.2), using the package logistf. The package uses the penalized log likelihood ratio test as a default. As this method is also recommended by Heinze and Schemper (2002) for the binary logistic regression with maximum likelihood estimation, the penalized log likelihood ratio test is used. 
The data analysis starts with a multicollinearity test. Based on the results of the multicollinearity tests, binary logistic regressions are performed. At first, the influence of all independent variables on the acceptance levels of the selected gain sharing methods is investigated. This is followed by an analysis of the influence of behavioral aspects on the acceptance level of each partner separately. For this, three additional logistic regressions, one for manufacturers, one for LSPs and one for retailers, are performed. Through an extensive comparison between the logistic regressions, differences in the parties' acceptance levels and the influence of behavioral aspects are identified. In Figure 3.2, an overview of the procedure of the data analysis is presented. 


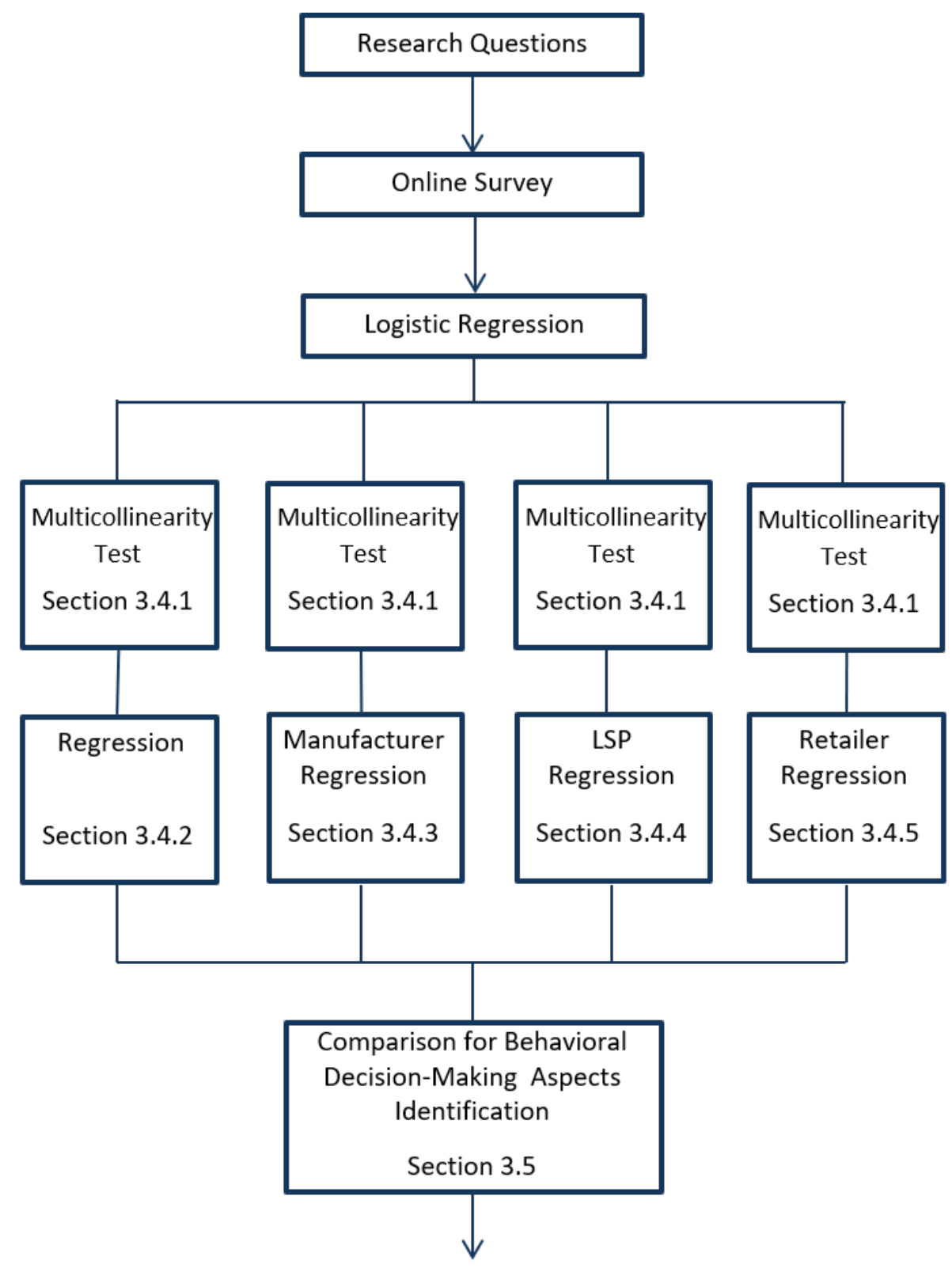

Research Output

Figure 3.2: Procedure of the data analysis 


\subsection{Statistical Analysis and Results}

In the following, the statistical analysis and results are presented. At first, the results of the multicollinearity test are outlined. This is followed by outcomes of the logistic regression, in which the influence of all independent variables on the acceptance levels is observed. Next, results for each collaborative party are shown separately. In the remainder of this section, a significance level of $5 \%$ is taken as the standard significance level.

\subsubsection{Multicollinearity}

One common problem when using multiple independent variables in a logistic regression is the occurrence of correlations among independent variables. A strong correlation between two independent variables leads to a problem known as multicollinearity. This can seriously distort the interpretation of the model (Greene, 2003). Table 3.4 shows the correlation matrix of the independent variables used in the logistic regression. All correlation coefficients have small values $(\leq 0.5)$, indicating no problems with multicollinearity. Consequently, all independent variables are included in the logistic regressions. 


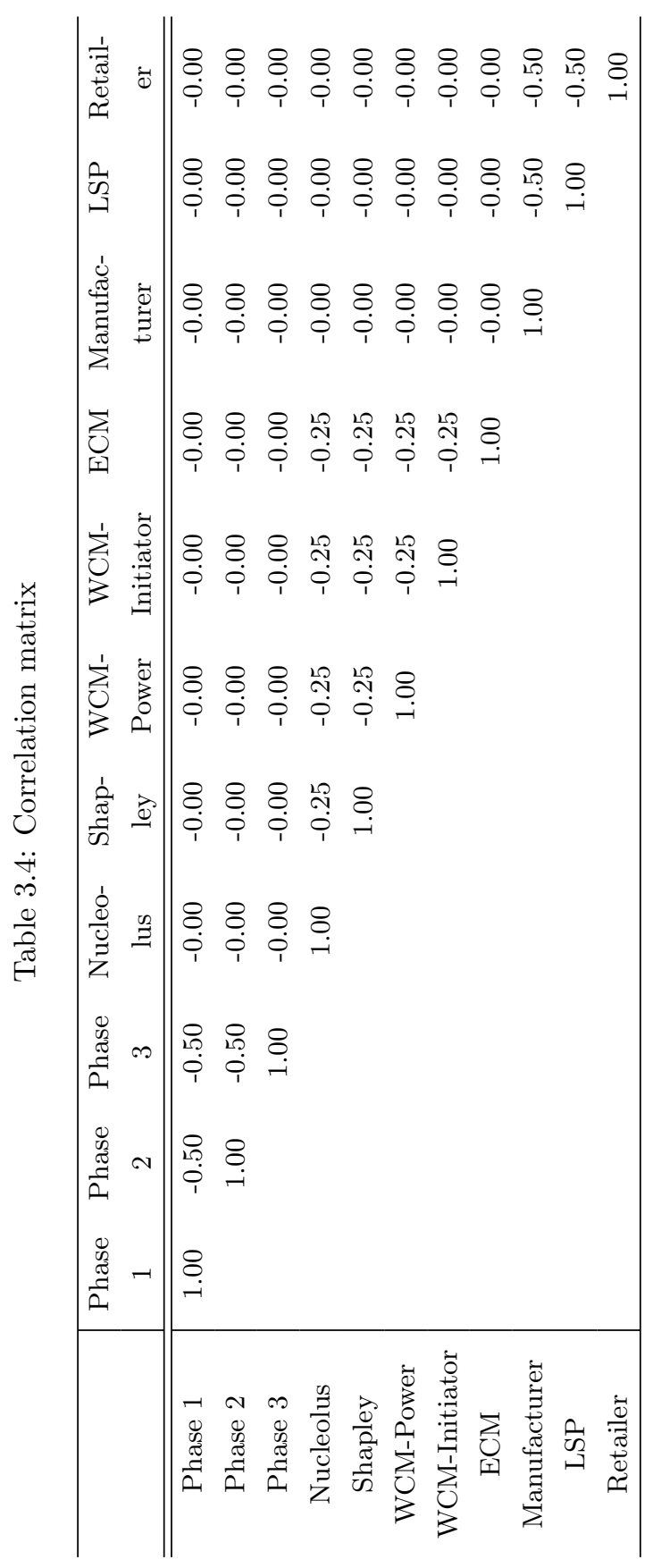




\subsubsection{Regression}

In this section, acceptance levels of the gain sharing methods are investigated. In Figure 3.3, the acceptance levels of the three parties over all gain sharing methods and phases are displayed. The overall business practitioners' acceptance level is $54.44 \%$. Looking at each partner individually, LSPs show the highest level of acceptance, while retailers show the lowest. This is confirmed by the logistics regression, see Table 3.5. The coefficient of the LSPs is positive, indicating that in comparison to manufacturers, the LSPs have a significantly higher level of acceptance even at a $1 \%$ significance level. In contrast, the retailers show a negative coefficient, indicating a significantly lower acceptance level in comparison to the manufacturers even at a $1 \%$ significance level.

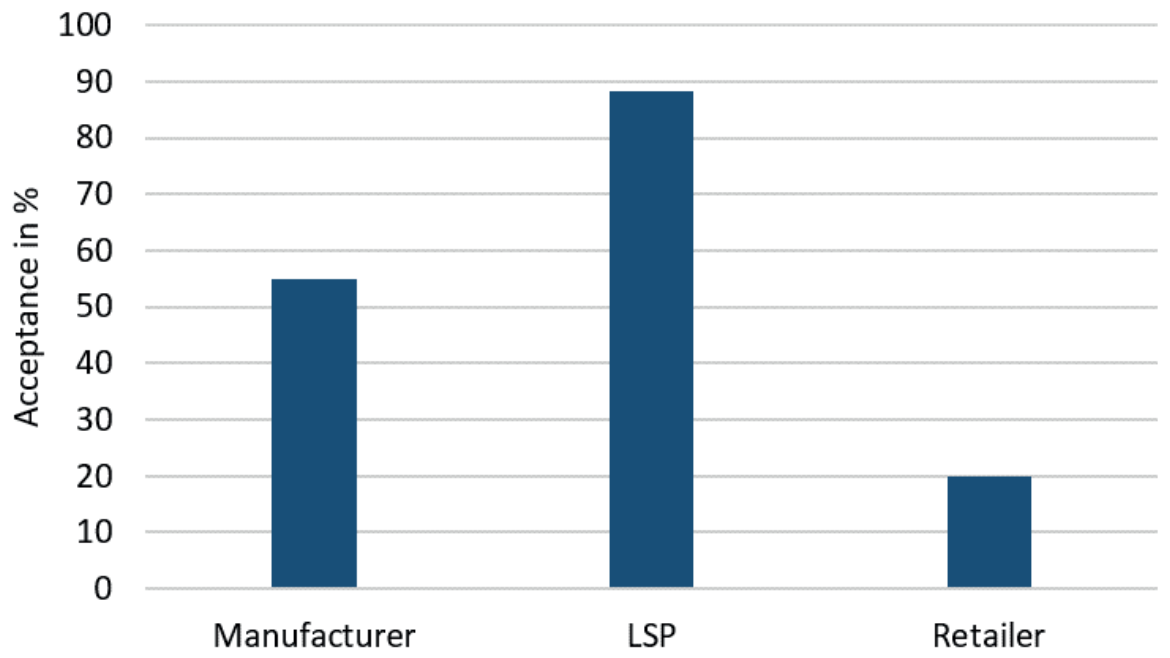

Figure 3.3: Acceptance levels of each collaborative party

In Figure 3.4, the acceptance levels of the five gain sharing methods in each phase are displayed. For the collaborative parties, the Nucleolus reveals the smallest level of acceptance with an average of $44.44 \%$ over three phases. This is also observed by the logistic regression, see Table 3.5. All allocation methods show a positive coefficient, indicating that, compared to the Nucleolus, all other methods have a higher level of acceptance. However, only the ECM shows a significant increase in the acceptance levels. Taking the phases into account, no significant difference between the phases is observed, see Table 3.5. 


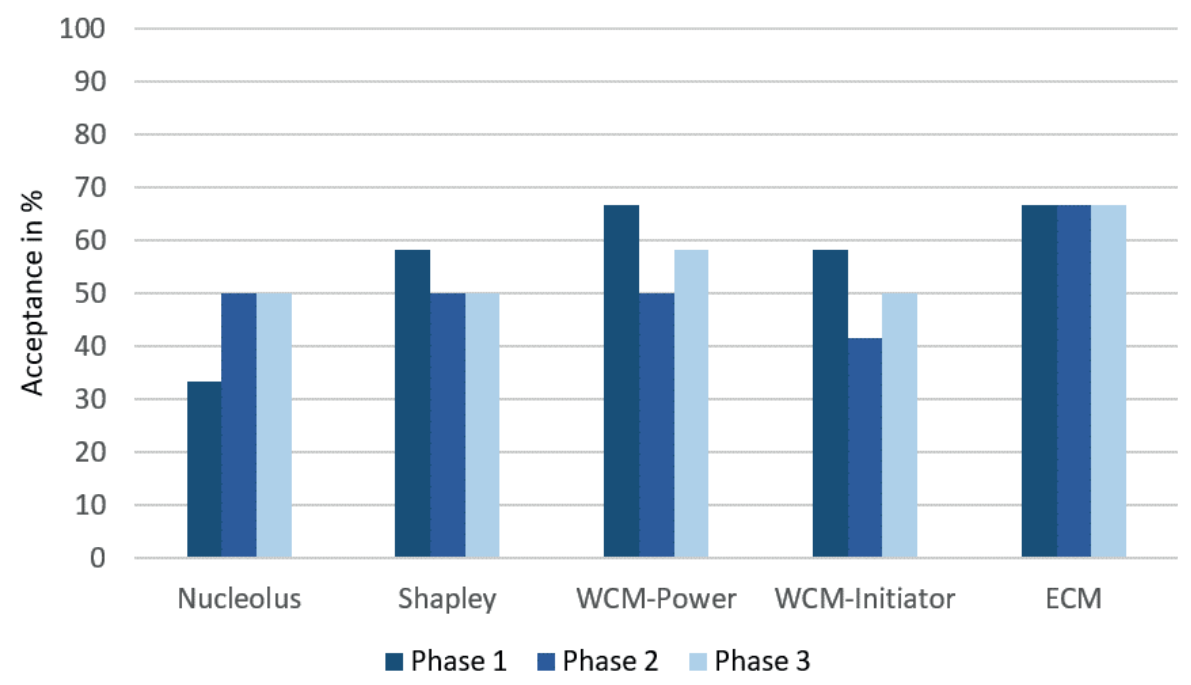

Figure 3.4: Acceptance levels of the five different gain sharing methods and the three different phases

Table 3.5: Logistic regression output for all collaborative parties

\begin{tabular}{c|cc}
\hline & Coefficient & Significance \\
\hline \hline Intercept & -.242 & .633 \\
Shapley & .474 & .399 \\
WCM-Power & .790 & .161 \\
WCM-Initiator & .317 & .574 \\
ECM & 1.274 & .025 \\
Phase 2 & -.287 & .512 \\
Phase 3 & -.096 & .827 \\
LSP & 1.769 & .000 \\
Retailer & -1.564 & .000 \\
\hline
\end{tabular}

\subsubsection{Regression Manufacturer}

In Figure 3.5, the manufacturers' acceptance levels of the five gain sharing methods in each phase are displayed. For the manufacturers, the overall level of acceptance is $55 \%$. Huge differences in the acceptance levels between the methods can be observed. Moreover, an increase in acceptance from the Nucleolus to the ECM 
is noticeable. Therefore, the Nucleolus is the least accepted method with $8.33 \%$, averaged over all three phases. On the contrary, the most accepted method with an acceptance level of $100 \%$ is the ECM. In Table 3.6, the results of the logistic regression are presented. A significant increase in the acceptance levels for all other gain sharing methods compared to the Nucleolus is identified. Furthermore, a significant influence of the information availability on the acceptance levels is observed. Compared to Phase 1, the acceptance levels of Phase 2 and Phase 3 are significantly lower, with the lowest level of acceptance in Phase 2.

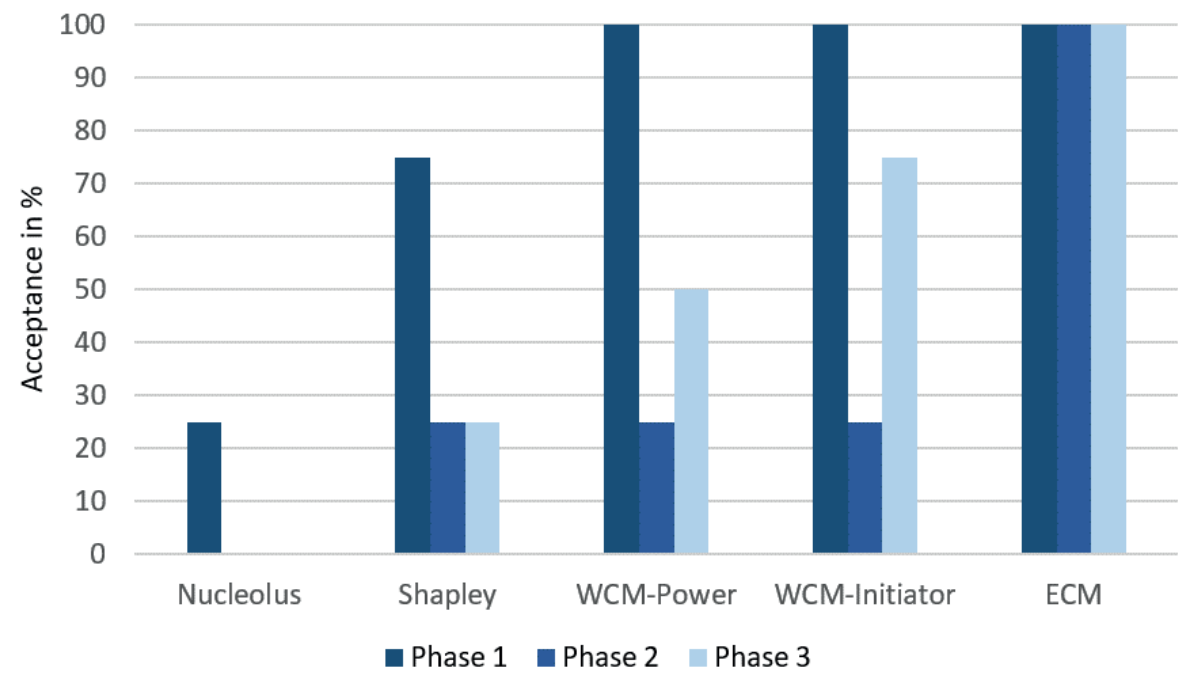

Figure 3.5: Acceptance levels of the five gain sharing methods and the three phases for the manufacturers

Table 3.6: Logistic regression output for the manufacturers

\begin{tabular}{c|cc}
\hline & Coefficient & Significance \\
\hline \hline Intercept & -.944 & .292 \\
Shapley & 2.355 & .037 \\
WCM-Power & 3.239 & .003 \\
WCM-Initiator & 3.665 & .001 \\
ECM & 6.517 & .000 \\
Phase 2 & -3.152 & .000 \\
Phase 3 & -2.171 & .015 \\
\hline
\end{tabular}




\subsubsection{Regression LSP}

In Figure 3.6, the LSPs' acceptance levels of the five gain sharing methods in each phase are displayed. The LSPs show a high overall acceptance level of $88.33 \%$. Moreover, for each allocation method acceptance levels of $75 \%$, averaged over the three phases, and higher are identified. Furthermore, differences in the acceptance levels of the phases are observed. A significant influence of the information availability on the acceptance levels is identified at a $10 \%$ significance level, see Table 3.7.

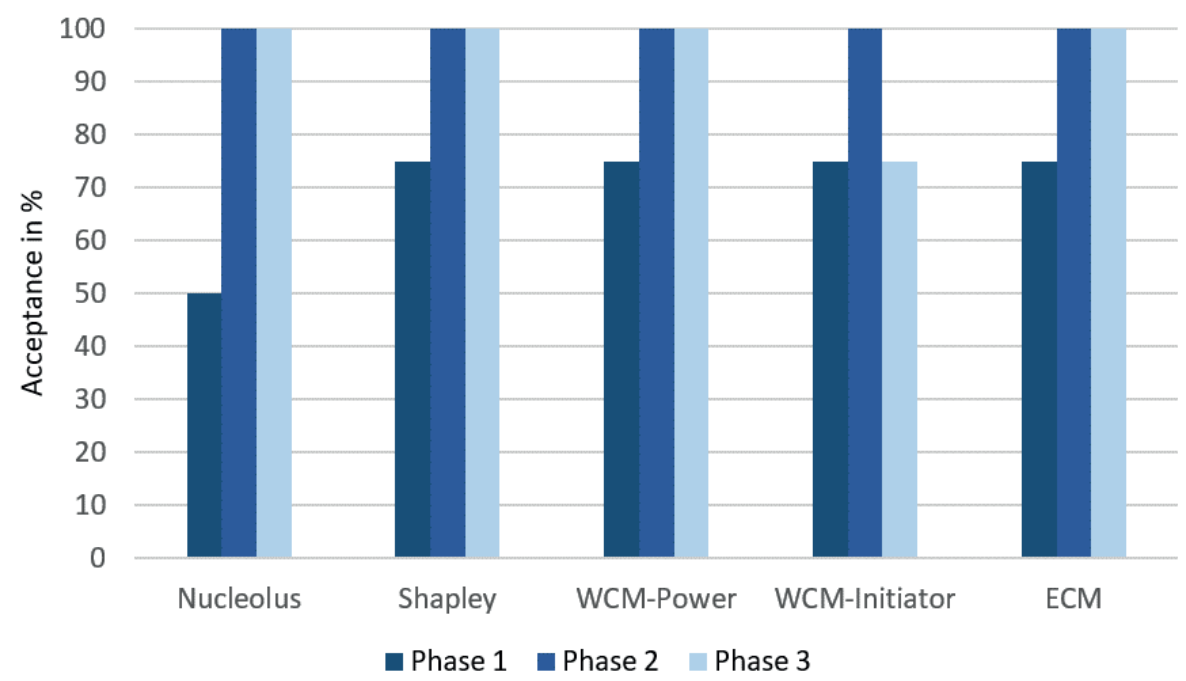

Figure 3.6: Acceptance levels of the five gain sharing methods and the three phases for the LSPs

Table 3.7: Logistic regression output for the LSPs

\begin{tabular}{c|cc}
\hline & Coefficient & Significance \\
\hline \hline Intercept & .296 & .719 \\
Shapley & .710 & .553 \\
WCM-Power & .710 & .553 \\
WCM-Initiator & .000 & 1.000 \\
ECM & .710 & .553 \\
Phase 2 & 2.757 & .010 \\
Phase 3 & 1.644 & .050 \\
\hline
\end{tabular}




\subsubsection{Regression Retailer}

Figure 3.7 displays the retailers' acceptance levels of the five gain sharing methods in each phase. In general the retailers have a low level of acceptance. They show a decrease in the acceptance from the Nucleolus to the ECM, with the lowest level of acceptance for the WCM-Initiator, which is not accepted. The highest acceptance level can be assigned to the Nucleolus with on average of $41.67 \%$ over the three phases. Furthermore, a significantly lower acceptance compared to the Nucleolus is identified for the WCM-Initiator and the ECM at a $10 \%$ significance level. Overall, no significant influence of the phases is observed, see Table 3.8.

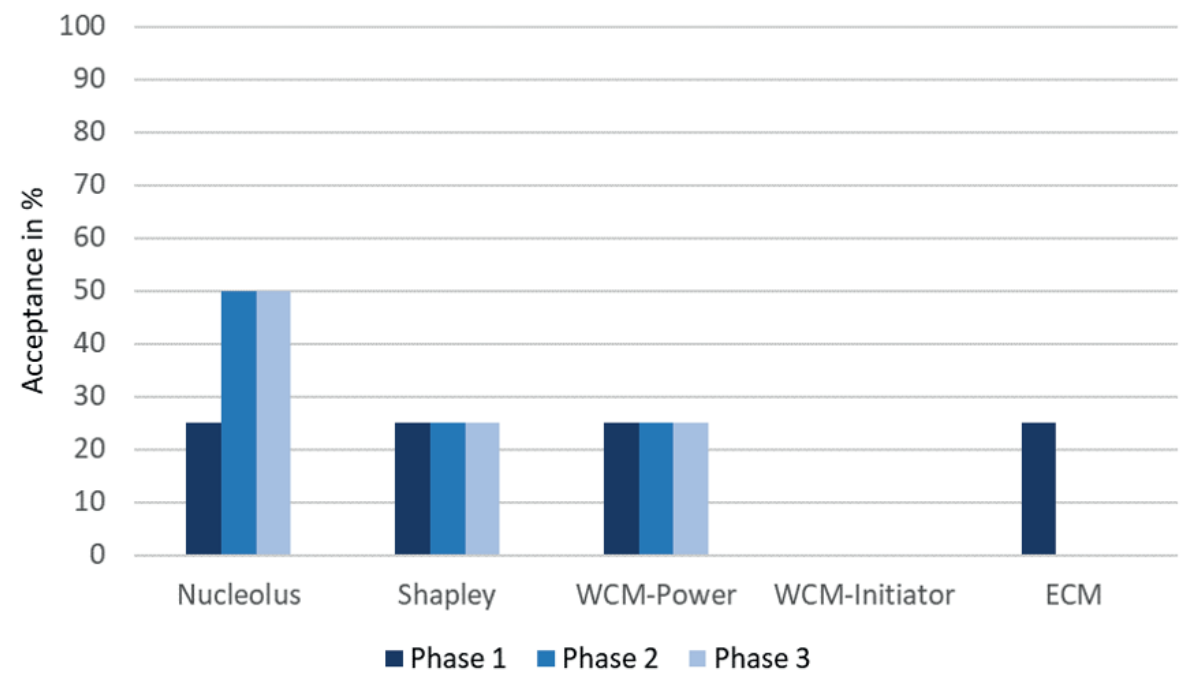

Figure 3.7: Acceptance levels of the five gain sharing methods and the three phases for the retailers 
Table 3.8: Logistic regression output for the retailers

\begin{tabular}{c|cc}
\hline & Coefficient & Significance \\
\hline \hline Intercept & -.296 & .675 \\
Shapley & -.659 & .418 \\
WCM-Power & -.659 & .418 \\
WCM-Initiator & -2.830 & .014 \\
ECM & -1.666 & .075 \\
Phase 2 & .000 & 1.000 \\
Phase 3 & .000 & 1.000 \\
\hline
\end{tabular}

\subsection{Result Discussion}

The case studies reveal two main findings. First, the parties' acceptance of the gain sharing method is influenced by the available information and cognitive biases. Second, each partner is individually influenced by the information availability and different parties reveal various cognitive biases. As a result of the individual influence of information availability and varying cognitive biases, no allocation method is accepted by all collaborative parties. In the following, the influence of available information and cognitive biases on the decision to accept is outlined for each party. In addition, for each party separately and for all participants together, the preferred gain sharing method, which results from the influence of behavioral aspects, is identified.

The results show a significant influence of the information availability for manufacturers and LSPs. However, the manufacturers are negatively influenced by the information availability, whereas the LSPs in the Dutch FMCG industry are positively influenced. For the manufacturers, a significantly negative influence was observed for Phase 2, even at a $1 \%$ significance level. In this phase, the manufacturers received the information that the lowest gain share had been assigned to them, omitting the fact that they are the smallest player in the supply chain. The information was only provided in Phase 3, which increased the acceptance level compared to Phase 2. Nevertheless, the acceptance level of Phase 3 was lower than in Phase 1. Unlike the manufacturers, the LSPs in the Dutch FMCG industry were significantly positively influenced by the available information. On the contrary, no significant influence of information availability could be identified 
for the retailers.

In addition to the influence of an information availability, the influence of cognitive biases could be identified for all parties. With regard to manufacturers' acceptance levels for the Nucleolus, there was only one manufacturer who accepted the gain share assigned by the Nucleolus in Phase 1. The allocation method was rejected by all other business practitioners, even though the assigned gain share of $4,333.33 €$ was greater compared to the manufacturer's contribution of $-5,000 €$. Consequently, an impact of cognitive biases can be assumed for business practitioners. The manufacturers had to invest the highest amount for the launch of the SCC, see Table 3.2. Taking this into account, the manufacturers' reason for rejecting the gain sharing methods might be the small share of the gain, that cannot justify the costs and efforts. The LSPs in the Dutch FMCG industry show the highest acceptance. This can be explained by the influence of a cognitive bias, the so-called choice-supportive bias (Mather and Johnson, 2000). The LSPs are usually the ones initiating the collaborations in the Dutch FMCG industry. Therefore, no matter which gain share is assigned to them, the LSPs always show a high acceptance level. On the contrary, the retailers reveal a very low acceptance level of $20 \%$. The low acceptance level of the retailers can be explained by their high contribution to the coalition gain combined with a generally lower gain in all gain sharing methods, see Tables 3.2 and 3.3. The missing influence of the information availability on the acceptance levels indicates an additional influence of cognitive biases. One explanation might be the powerful position of the retailers in the Dutch FMCG supply chain, identified in Chapter 2. Taking into account the powerful position of the retailers, this party might demand a bigger portion of the gain. Tijs and Driessen (1986) also outline that the choice of the method depends on the parties' feelings of power. The WCM-Power already considered the parties' positions of power; the highest weight was assigned to the retailers. However, also this allocation method revealed a low acceptance. Based on this result, it is assumed that the weight did not fully represent the retailers' power in the Dutch FMCG industry.

The above-mentioned findings clearly show that the different parties are not influenced by the available information in the same way and that different parties show various cognitive biases. Due to the individual influence of information availability and varying cognitive biases, no allocation method is preferred by all collaborative parties. The manufacturers preferred the ECM with an acceptance 
level of $100 \%$. In contrast, the retailers had a clear preference for the Nucleolus with $41.67 \%$ and the LSPs showed equal acceptance for the Shapley value, the WCM-Power and the ECM. In addition, the overall acceptance of all parties combined for the different gain sharing methods revealed only minor differences, and these acceptance levels were far from 100\%. With an acceptance level of $66.67 \%$, the business practitioners showed a slight preference for the ECM. These findings confirm the result from the literature that no gain sharing method is preferred by all collaborative parties (Tijs and Driessen, 1986; Vanovermeire et al., 2014) and demonstrate the challenge of applying a gain sharing method that is accepted by all collaborative parties, which is, however, essential for the implementation and success of an SCC (Cruijssen et al., 2007a; Crujssen and ArgusI, 2012; Leng and Parlar, 2009).

\subsection{Conclusion, Implications and Future Research}

In this chapter, one of the most important SCC challenges, the allocation of the coalition gain, has been investigated. For this purpose, the acceptance levels of selected gain sharing methods have been observed in vertical three-echelon SCCs in the Dutch FMCG industry. In addition, in order to identify the cause of the acceptance or rejection of an assigned gain share, the influence of behavioral decisionmaking aspects, namely information availability and cognitive biases, on the acceptance decision has been examined. The results indicate that the acceptance of a gain sharing method depends on available information and cognitive biases. Furthermore, due to different influences of information availability and varying cognitive biases, no allocation method is accepted by all collaborative parties.

\subsubsection{Theoretical Implications}

This chapter is an extension of the works by Cruijssen et al. (2007a) and Leng and Parlar (2009). In their study, Cruijssen et al. (2007a) identify the need for a fair gain allocation for the implementation and success of horizontal SCCs. In the context of vertical collaborations, Leng and Parlar (2009) confirm the importance of a fair allocation method for parties to stay in the SCC. Although the acceptance of and satisfaction with a gain sharing method is necessary for a sustainable collaboration, until now, the acceptance of these gain sharing methods in practice has not been examined. This chapter tries to fill this gap and thus enriches the SCM 
literature through the investigation of the acceptance levels of selected gain sharing methods in vertical three-echelon SCCs in the Dutch FMCG industry. Another contribution to the SCM literature is the integration of behavioral decision-making research. For a long time, the predominant assumption was that decision-makers are thinking rationally. However, decision-makers are human beings and therefore their decisions are influenced by bounded rationality and cognitive biases (Schenk, 2011; Simon, 1979; Sterman, 1989). To ensure practical validity, it is necessary to incorporate behavioral research in studies (Tokar, 2010). In this chapter, two behavioral aspects, namely information availability and cognitive biases, were taken into account and therefore novel insights in the SCC's allocation challenges are provided.

\subsubsection{Practical Implications}

Results show that providing the same information to all collaborative parties in the Dutch FMCG industry would lead to unsatisfactory gain allocations. As stated by Cruijssen et al. (2007a), a gain sharing method which is perceived as fair and is accepted by all collaborative parties is crucial for the implementation and success of the SCC. As a result, one practical implication to overcome the barrier is to provide all relevant information for each party individually. Furthermore, differences between the different parties indicated the influence of various cognitive biases. Cognitive biases influence our rational behavior resulting in unpredictable decisions (Schenk, 2011). Therefore, in order to increase the predictability of the behavior, one idea based on research by Soll et al. (2014) is to apply so-called debiasing-techniques. One debiasing-technique is to provide all relevant information packaged in an intuitively comprehensible and compelling format. A suggestion is to show a figure as a graph, which provides clear and relevant information about the gain allocation.

\subsubsection{Further Research Directions}

This chapter offers several opportunities for further research. The small sample size represents one limitation of the quantitative case study research. This is balanced by the experience and expertise with regard to the SCC of the respondent base. Furthermore, the results support statements from the literature that there is no gain sharing method which is accepted by all collaborative parties, (e.g Tijs and Driessen, 1986) and that decision-makers are influenced by available information 
and cognitive biases (Sterman, 1989). Therefore, it is assumed that surveys with a greater sample size, in other industries and/or geographical areas will confirm the findings of this study. Moreover, the participants of the online survey were confronted with one specific artificial situation without e.g. monetary incentives. Conducting the online survey in a real-life situation could identify other important behavioral decision-making aspects. Furthermore, additional allocation methods and weights can be considered. In the study, four selected allocation methods have been tested; further research could also include gain sharing methods like the EPM or the WRSM in the survey. In addition, two weights, determined based on interviews, were taken into account in the survey. For the retailer, it has been identified that the chosen weight for the WCM-Power is no adequate representation for its powerful position. Further research should therefore take into account other important aspects of the FMCG industry and/or other industries as well as vary the weights assigned to the parties. Moreover, two behavioral decisionmaking aspects were taken into account. In addition, debiasing-techniques have been proposed. Additional research could also take other aspects into consideration, for instance the availability of time, which is another component of the bounded rationality mentioned by Simon (1979). The lack of available time forces people to use heuristics or cognitive biases (Schenk, 2011; Simon, 1979). Future surveys should also integrate the availability of time by conducting the surveys in a controlled environment where participants only have a predetermined time to make a decision. Furthermore, debiasing-techniques should be tested in practice. Finally, it could be identified that due to the different influences of information availability and varying cognitive biases no gain sharing method is accepted by and satisfies all collaborative parties. Developing allocation methods that focus on the parties' acceptance of and satisfaction with the assigned gain share might be one option to deal with the outlined problem. 


\section{A Preliminary Study}

The preliminary study consisted of 20 companies including seven manufacturers, six LSPs and seven retailers from the Dutch FMCG industry. For the data collection, individual semi-structured interviews were conducted mostly face-to-face with the supply chain managers from the companies. The following questions concerning the gain sharing methods were asked to the interviewees:

- What does "fair gain sharing" mean to you and your company?

- To what extent are you willing to share gains among the entire supply chain? (answer on a 5-point Likert scale)

- Would it be a problem for your company to share gains, that are achieved by your company, but are a result of a collaboration with other collaborative parties? To what extent and why?

- In your experience, how do other parties within your supply chain react to gain sharing?

- Before you launch a collaboration, is the transparency of how much each party needs to invest in collaborations an important issue?

- Before you launch a collaboration, is it crucial information for you to know how parties will benefit? To what extent and why? 


\section{B Online Survey}

In the following an example of the online surveys is presented. The online survey is one for a participant party $\mathrm{A}$, the manufacturer.

\section{Behavioral Study - Party A}

$1 / 23$ $4 \%$

Welcome to this behavioral study.

Please be sure to answer the questions the same way you would act in a real-life situation. The questionnaire will take you through different scenarios. More information will be presented as the questionnaire progresses. Please note that it will not be possible to go back once a question is answered.

Thank you for participating in our survey.

Your role: Party $A$

Figure 3.8: Example of the online survey

\section{Behavioral Study - Party A}

$3 / 23$

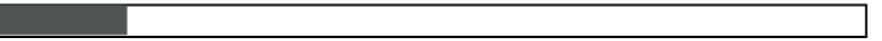

$13 \%$

\section{Collaboration Project X}

Imagine you are party $A$ in a vertical supply chain that involves three different parties (party A, party B and party C). A new opportunity has presented itself in the form of a collaboration project: Project $\mathrm{X}$.

The goal of this project is to take advantage of the extra benefits resulting from the collaboration by getting different parties (party A, party B, party C) involved to work together and optimize supply chain operations. It is expected that the parties involved require different investments and the distribution of gains over the supply chain will not be proportional.

You will be presented with your individual benefits (gains), costs and profits and with the overall supply chain profit once all parties enter the collaboration. Please investigate this information carefully.

Figure 3.9: Example of the online survey

Figure 3.10 to Figure 3.12 show examples for the part of the online survey belonging to Phase 1. In Figure 3.12 an example for the question in Phase 1 is 
shown. In the online survey in total five questions were asked; one for each gain sharing method.

\section{Behavioral Study - Party A}

$4 / 23$

$17 \%$

\section{Collaboration Project X}

\begin{tabular}{|l|c|c|}
\hline & Party A & Party B \\
\hline Benefits (expected gains) & $80,000 €$ & Party C \\
\hline Costs (investments) & $85,000 €$ \\
\hline Direct profit & $-5,000 €$ & \\
\hline Overall collaboration profit & & \\
\hline
\end{tabular}

The benefit (expected gain) and costs (investments) presented are your individual direct benefits and investments for the project. The profit presented would be your direct profit in case no gain sharing methods are applied. The overall collaboration profit is the sum of all individual direct profits within the supply chain.

On the following pages, different gain sharing methods will be applied and a portion of the collaboration profit will be assigned to you. This assigned profit will replace your direct profit in the case of gain sharing.

Figure 3.10: Example of the online survey

\section{Behavioral Study - Party A}

$5 / 23$

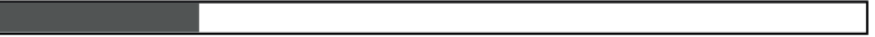

$22 \%$

\section{Collaboration Project X}

Now you will be presented with different allocation scenarios for the supply chain profit resulting from the collaboration project. The allocation is the result of gain sharing methods that have been applied.

Please indicate whether you would accept or reject the presented solution.

Figure 3.11: Example of the online survey 


\section{Behavioral Study - Party A}

$6 / 23$ $26 \%$

\begin{tabular}{|l|c|c|c|}
\hline & Party A & Party B \\
\hline Benefits (expected gains) & $80,000 €$ & Party C \\
\hline Costs (investments) & $85,000 €$ & \\
\hline Direct profit & $-5,000 €$ & \\
\hline Overall collaboration profit & & \\
\hline
\end{tabular}

Would you accept the following portion of the overall collaboration profit (replacing your direct profit)?

$4,333 €$
O Accept
O Reject

Figure 3.12: Example of the online survey

Figure 3.13 and Figure 3.14 show examples for the part of the online survey belonging to Phase 2. In Figure 3.14 an example for the question in Phase 2 is shown. In the online survey in total five questions were asked; one for each gain sharing method.

\section{Behavioral Study - Party A}

$11 / 23$ $45 \%$

\section{Collaboration Project X}

Now you will be presented with the same allocation scenarios for the supply chain profit resulting from the collaboration project; however, you will get a transparent overview of the benefits (gains), costs and profits of the other parties involved. The allocation is the result of the gain sharing methods that have been applied.

Please indicate whether you would accept or reject the presented solution.

Figure 3.13: Example of the online survey 
Behavioral Study - Party A

$$
12 / 23
$$

\begin{tabular}{|l|c|c|c|}
\hline & Party A & Party B & Party C \\
\hline Benefits (expected gains) & $80,000 €$ & $50,000 €$ & $250,000 €$ \\
\hline Costs (investments) & $85,000 €$ & $10,000 €$ & $80,000 €$ \\
\hline Direct profit & $-5,000 €$ & $40,000 €$ & $170,000 €$ \\
\hline Profit allocation & $4,333 €$ & $55,333 €$ & $145,333 €$ \\
\hline Overall collaboration profit & \multicolumn{3}{|c|}{$205,000 €$} \\
\hline
\end{tabular}

Would you accept the following portion of the overall collaboration profit (replacing your direct profit)?

$4,333 €$
O Accept
O Reject

Figure 3.14: Example of the online survey

Figure 3.15 to Figure 3.17 show examples for the part of the online survey belonging to Phase 3. In Figure 3.17 an example for the question in Phase 3 is shown. In the online survey in total five questions were asked; one for each gain sharing method.

\section{Behavioral Study - Party A}

$17 / 23$ $74 \%$

\section{Collaboration Project X}

Now you will be presented with the same allocation scenarios for the supply chain profit resulting from the collaboration project; however, you now not only get a transparent overview of the benefits (gains), costs and profits of the other parties involved, but also an idea of the market conditions. The allocation is the result of gain sharing methods that have been applied.

Please indicate whether you would accept or reject the presented solution.

Figure 3.15: Example of the online survey 


\section{Behavioral Study - Party A}

$$
18 / 23
$$
$78 \%$

\section{Project X Assumptions}

Party $C$ is the biggest player on the market with a market share of $25 \%$ and is a member of a union called "Super Unie". Relationships with this party are important.

Party $\mathrm{B}$ is a medium player on the market with a market share of $10 \%$.

Party $\mathrm{A}$ is based locally (not international) and the goods are relatively easy to substitute. Party $A$ is not a member of any association or union of parties from the same position in the supply chain.

Figure 3.16: Example of the online survey

\section{Behavioral Study - Party A}

$19 / 23$

\begin{tabular}{|l|c|c|c|}
\hline & Party A & Party B & Party C \\
\hline $\begin{array}{l}\text { Benefits (expected } \\
\text { gains) }\end{array}$ & $80,000 €$ & $50,000 €$ & $250,000 €$ \\
\hline $\begin{array}{l}\text { Costs } \\
\text { (investments) }\end{array}$ & $85,000 €$ & $10,000 €$ & $80,000 €$ \\
\hline Direct profit & $-5,000 €$ & $40,000 €$ & $170,000 €$ \\
\hline Profit allocation & $4,333 €$ & $55,333 €$ & $145,333 €$ \\
\hline $\begin{array}{l}\text { Overall } \\
\text { collaboration profit }\end{array}$ & \multicolumn{3}{|c|}{$205,000 €$} \\
\hline
\end{tabular}

Party $C$ is the biggest player on the market with a market share of $25 \%$ and is a member of a union called "Super Unie". Relationships with this party are important.

Party $B$ is a medium player on the market with a market share of $10 \%$.

Party $A$ is based locally and the goods are relatively easy to substitute. Party $A$ is no member of any association or union of parties from the same position in the supply chain.

Would you accept the following portion of the overall collaboration profit (replacing your direct profit)?

\section{$4,333 €$}
O Accept
O Reject

Figure 3.17: Example of the online survey 



\section{Chapter 4}

\section{A Comprehensive Gain}

\section{Sharing System Maximizing}

\section{Satisfaction in Supply Chain} Collaborations $^{1}$

\subsection{Introduction}

This chapter builds upon the research findings of Chapter 3. As outlined in Chapter 3, no gain sharing method is accepted by and satisfies all collaborative parties, which is however essential for the implementation and success of SCCs. In addition, the preliminary study with collaborative parties from the Dutch FMCG industry, which is provided in Appendix 3A, indicated that parties do not see the need for complex gain sharing methods. In practice, simple rules that are easy to understand are preferred (interview with one LSP on 13.05.2015; interview with one manufacturer on 28.05.2015). This is stressed by Cruijssen et al. (2007b) as well as Leng and Parlar (2005), who outline that mathematical simplicity, applicability and transparency constitute key allocation characteristics in practice. Furthermore, the literature overview in Section 3.2.1 demonstrates the presence of a wide range of gain sharing methods. As each method has its specific advantages

\footnotetext{
${ }^{1}$ This chapter is based on the paper Grigoriev et al. (2019b), submitted for publication.
} 
and drawbacks, it remains ambiguous which technique should be applied in an SCC comprised of parties with different objectives.

Since mathematical simplicity, applicability and transparency constitute key allocation characteristics in practice, in this chapter a simple gain sharing method is introduced. To ensure an allocation's simplicity, its intuitive understanding and a fair acceptance, the new gain sharing system focuses on the maximization of the parties' satisfaction. To the best of the author's knowledge, no gain sharing method has focused on all these criteria together. Furthermore, the gain sharing system adds value to the current gain allocation research by providing managers with a complete gain sharing scheme which requires limited input data in order to provide robust output on the gain sharing decision as well as with related useful key performance indicators (KPIs). Moreover, the majority of SCC publications focus on the application of gain sharing methods in horizontal collaborations (Guardiola et al., 2007). In this chapter, the proposed allocation system is applied to a vertical SCC. In addition, except for the paper by Leng and Parlar (2009), all articles discuss gain sharing methods applied to only two-echelon supply chains. This study considers a three-level vertical SCC between one manufacturer, one LSP and one retailer. The essential contribution of this work is twofold. On the one hand, this research is the first to identify a gain sharing approach focusing on the parties' satisfaction. On the other hand, the gain sharing decision is supported by providing practitioners with a complete, simple and robust gain sharing system.

The remainder of this chapter is structured as follows. Section 4.2 introduces the gain sharing system developed in this study, followed by the application of the system to a vertical SCC in the Dutch FMCG industry in Section 4.3. In Section 4.4, the system stability is discussed based on various general fairness properties and a sensitivity analysis is presented. Finally, Section 4.5 concludes the chapter with key findings, theoretical and managerial implications as well as further research directions.

\subsection{Gain Sharing System}

As was stressed in the introduction, practical appreciation requires a gain sharing system that is simple to understand and to use while producing a fair and robust allocation of the coalition gain. In this section, a comprehensive and simple gain sharing system is presented. The goal of the system is to maximize the satisfaction 
of all collaborative parties through a minimax regret approach. In Figure 4.1, the proposed gain sharing system is illustrated in a block diagram. The gain sharing system consists of three parts: input, gain sharing algorithm and output. Furthermore, the dynamic character of an SCC was taken into consideration. The input factors of the gain sharing system may change during the SCC. As a result, the gain sharing (re)allocation should be recomputed when necessary. In the following sections, the three system parts are explained in detail. Section 4.2.1 explains the input factors, followed by a clarification of the gain sharing algorithm in Section 4.2.2. Finally, the output is discussed in Section 4.2.3.

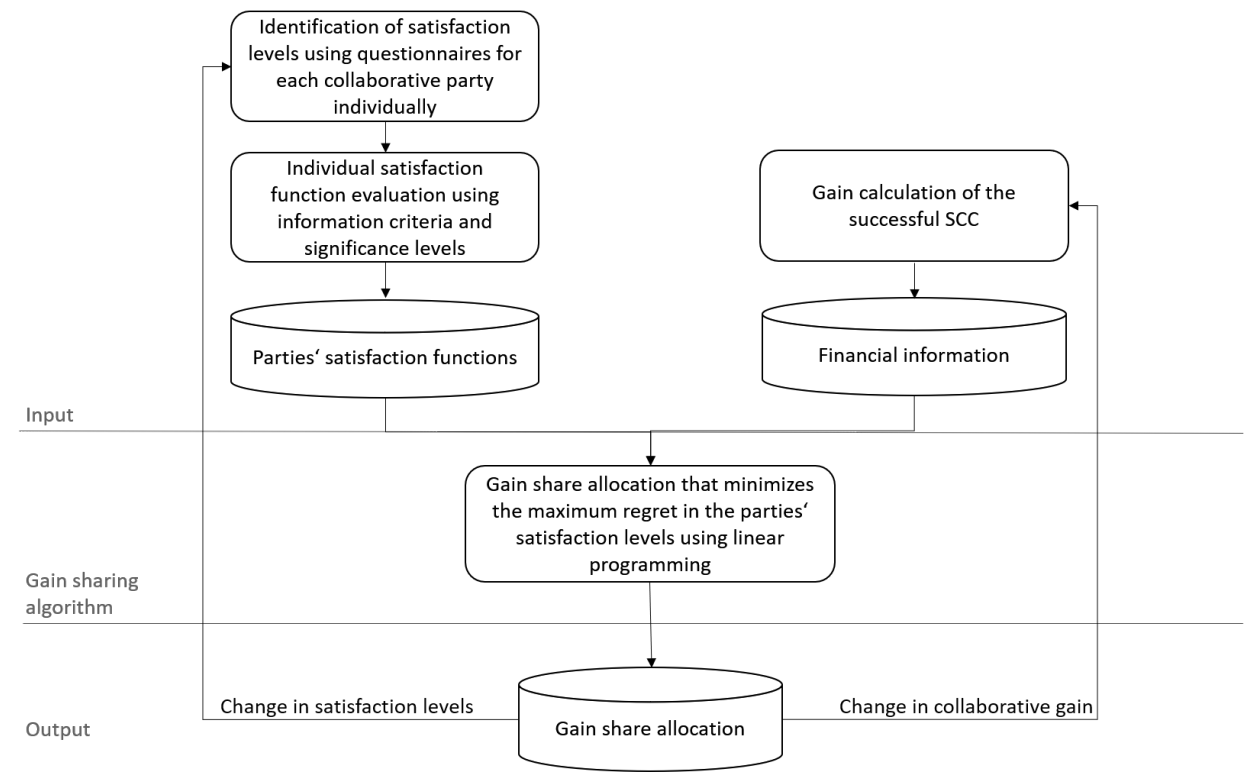

Figure 4.1: Block diagram of the comprehensive gain sharing system

\subsubsection{Input}

The gain sharing algorithm demands two input elements: the parties' satisfaction functions and the financial information of the SCC.

The literature proposes many definitions of the term satisfaction. One definition from Oliver (2014) is that "satisfaction is the customer's fulfillment response. It is a judgment that a product/service feature or the product or service itself provided (or is providing) a pleasurable level of consumption - related fulfillment, including levels of under- and overfulfillment". Lozano et al. (2013) define the 
satisfaction of a (sub)coalition in the context of gain sharing methods as the excess of cost savings of the grand coalition minus the total gain of a (sub)coalition. In this chapter, it is assumed that the party's satisfaction depends on the gain share that is assigned to this party. The author is aware that the gain share is not the only aspect that has an influence on the parties' satisfaction levels and therefore in Section 4.5 additional aspects that could be considered in further research are proposed. In order to derive the parties' satisfaction functions, the parties' satisfaction levels for various possible gain shares need to be identified. For this purpose, questionnaires need to be distributed to the collaborative parties. Using questionnaires as a research instrument is useful since they are usually inexpensive to administer, little training is required to develop them and they are easy and quick to analyze (Wilkinson and Birmingham, 2003). This contributes to the simplicity of the gain sharing system.

In the questionnaire, the parties are asked how satisfied they are with a certain gain share. An example question would be: "How satisfied are you with a gain share of $20 \%$ of the coalition gain?". The party's responses are elicited on a fivepoint Likert scale ranging from 'very dissatisfied' to 'very satisfied'. Using a Likert scale to measure the satisfaction is common practice. Examples are the works by Mueller and McCloskey (1990) as well as Traynor and Wade (1993), which both measure the job satisfaction of employees on a five-point Likert scale. In order to receive valid responses, it is necessary to indicate how many parties are involved in the SCC. After conducting the questionnaires, they are analyzed and the parties' satisfaction functions are derived from the data. To identify the most appropriate satisfaction function for each party individually, several non-linear regressions could be performed. For the purpose of narrowing down the possible functions, it is advisable to plot the data first. In order to evaluate the performance of the functions, not only the investigation of the significance levels of the coefficients are compared, but also the Akaike information criterion (AIC) (Akaike, 1974) and the Schwarz information criterion (SIC) (Schwarz et al., 1978). These two statistical criteria, which are both based on information theory, are often used when selecting the most appropriate model for underlying data (Sin and White, 1996). The function with the smallest AIC and SIC value should be preferred (Ludden et al., 1994). 
The second input factor is the financial information of the collaboration. Important information in this context is the overall coalition gain achieved by the grand coalition as well as the gain for each possible subcoalition.

\subsubsection{Gain Sharing Algorithm}

The gain sharing algorithm aims to increase the satisfaction of the collaborative parties through the minimization of the maximum regret. According to Loulou and Kanudia (1999) as well as Mausser and Laguna (1999), the minimax criterion is a reliable criterion for evaluating and selecting decisions under uncertainty and imperfect information. The minimax regret approach has been used to put more weight on the least satisfied party and thereby to increase the probability that no partner leaves the SCC, which in turn results in an increased probability of having a sustainable collaboration. In this study, the regret represents the difference between the best possible satisfaction level that is achieved when $100 \%$ of the gain is assigned to a party, and the actual satisfaction level of that party.

Let $N$ be the set of collaborative parties. For each partner $i \in N$, let $0 \leq x_{i} \leq 1$ denote the gain share of partner $i$ and let $s_{i}\left(x_{i}\right)$ represent the satisfaction level of partner $i \in N$ when $x_{i}$ share of the gain is allocated to $i$. Furthermore, $v(S)$ and $v(N)$ represent the gain share of a subcoalition $S \subset N, S \neq \emptyset$ and the gain share of the grand coalition, respectively. The following simple and intuitive gain sharing algorithm is proposed:

$$
\begin{aligned}
\min _{0 \leq x \leq 1} & \max _{i \in N}\left\{s_{i}(1)-s_{i}\left(x_{i}\right)\right\} \\
\text { s.t. } & \sum_{i \in N} x_{i}=1 \\
& \sum_{i \in S} x_{i} \geq v(S) / v(N)
\end{aligned}
$$

The objective function is to minimize the maximum regret of the collaborative parties. Constraints 4.2 and 4.3 ensure that the gain allocation is in the core and thus stable. As outlined in Section 3.2.1, being in the core guarantees that no party can increase its share/profit by leaving the grand coalition (Shapley, 1952). These constraints are included to ensure that there is no rational incentive for any party to leave the SCC. 


\subsubsection{Output}

The output of the gain sharing algorithm is the gain allocation, which minimizes the maximum parties' regret or, in other words, distributes the gain to satisfy all parties. In particular, the objective function represents one of the KPIs that will support managers to evaluate the performance of the gain sharing system. Other outputs are the satisfaction levels/functions for each party as well as the corresponding regrets.

\subsection{Implementation}

In this section, the proposed gain sharing system is applied to a vertical SCC between one manufacturer, one LSP and one retailer in the Dutch FMCG industry. The practical example is based on data provided by a Dutch logistics company specializing in efficient and sustainable solutions for supply chains. Note that the gain sharing system has been applied in order to theoretically illustrate the potential and high performance of the system. The practical implementation of the gain sharing system into an SCC is not part of this research. Furthermore, although the gain sharing system is applied to a vertical SCC the gain sharing system is not limited to vertical SCCs, but can easily be applied to horizontal or lateral SCCs as well.

\subsubsection{Satisfaction Functions and Financial Information}

In order to identify the most appropriate satisfaction function for the three parties individually, information about the satisfaction levels of various gain shares is needed for each party individually. For this purpose, results generated in the study introduced in Chapter 3 are used. In Chapter 3, among others the influence of the gain share on the parties' acceptance levels of selected gain sharing methods was examined in a vertical three-echelon SCC in the Dutch FMCG industry. Assuming that the acceptance levels are equal to the parties' satisfaction levels, the results of the study introduced in Chapter 3 were taken as a basis for the relationship between the assigned gain share and the satisfaction levels of the three parties. Based on this information, the satisfaction levels for the different gain shares were determined. For this purpose, a simulation tool was used. The satisfaction levels were simulated with 100 runs and 50 trials per run. Examples of the simulations are provided in Table 4.1. For various gain shares, the corresponding satisfaction 
levels for the three parties were displayed on a five-point Likert scale.

Table 4.1: Satisfaction levels of the three parties for varying gain shares

\begin{tabular}{c|ccc}
\hline $\begin{array}{c}\text { Gain Share } \\
\text { in } \%\end{array}$ & \multicolumn{3}{|c}{ Satisfaction level on Likert scale (1-5) } \\
Manufacturer & LSP & Retailer \\
\hline \hline 10 & 2 & 4 & 1 \\
20 & 3 & 4 & 1 \\
30 & 4 & 5 & 1 \\
40 & 5 & 5 & 2 \\
50 & 5 & 5 & 3 \\
60 & 5 & 5 & 3 \\
70 & 5 & 5 & 3 \\
80 & 5 & 5 & 4 \\
90 & 5 & 5 & 4 \\
100 & 5 & 5 & 5 \\
\hline
\end{tabular}

Plotting the data shown in Table 4.1 revealed the characteristic S-shape curve, also known as the sigmoid curve. In order to identify the most appropriate sigmoid function to represent the parties' satisfaction levels for the different gain shares, several non-linear regressions were performed using the software EViews 9 SV. EViews uses the Gauss-Newton algorithm as modified by Levenberg (1944) and Marquardt (1963). The straightforward logit model, also known as logit or logistics regression, turned out to be the best fit to represent the satisfaction of all collaborative parties (based on the significance levels, the AIC and the SIC):

$$
s_{i}\left(x_{i}\right)=\frac{a_{i}}{b_{i}+e^{c_{i} x_{i}}}
$$

where $x_{i}$ represents the gain share assigned to each partner $i=m, l, r$. Here, the index $m$ refers to the manufacturer, $l$ refers to the LSP and $r$ to the retailer. Coefficients $a, b$ and $c$ are determined by the non-linear regression. The corresponding outputs of the non-linear regressions for the three parties are depicted in Table 4.2. For all parties, the coefficients (reported in columns "Coef.") are highly significant at a $1 \%$ significance level, see columns "Prob.". 
Table 4.2: Output of the non-linear regression

\begin{tabular}{c|cc|cc|cc}
\hline & \multicolumn{2}{|c|}{ Manufacturer } & \multicolumn{2}{c|}{ LSP } & \multicolumn{2}{c}{ Retailer } \\
& Coef. & Prob. & Coef. & Prob. & Coef. & Prob. \\
\hline \hline$a_{i}$ & 1.23 & .000 & 8.48 & .000 & 0.72 & .000 \\
$b_{i}$ & 0.24 & .000 & 1.69 & .000 & 0.13 & .000 \\
$c_{i}$ & -9 & .000 & -9 & .000 & -3.5 & .000 \\
\hline
\end{tabular}

Figure 4.2 shows the plotted satisfaction functions. The LSP is the party which is most easily satisfied. As outlined in Chapter 3, the LSP is influenced by a cognitive bias; the so-called choice-supportive bias. Here, people tend to think positively about a decision they made, even if the decision has a flaw (Mather and Johnson, 2000). In the Dutch FMCG industry, the LSP often acts as the initiator for the launch of an SCCs. As a result, no matter what gain share is assigned to the LSP, this party will always be satisfied. In contrast, retailers have typically very low acceptance/satisfaction levels. Even if the largest portion of the gain is assigned to the retailers, this party is not satisfied, which might be a result of the powerful position of the retailer in the Dutch FMCG industry as outlined in Chapter 2. Regarding the manufacturer, the satisfaction function shows a steep increase from the beginning until a gain share of around $50 \%$ is received. Above that amount, the manufacturer is generally satisfied.

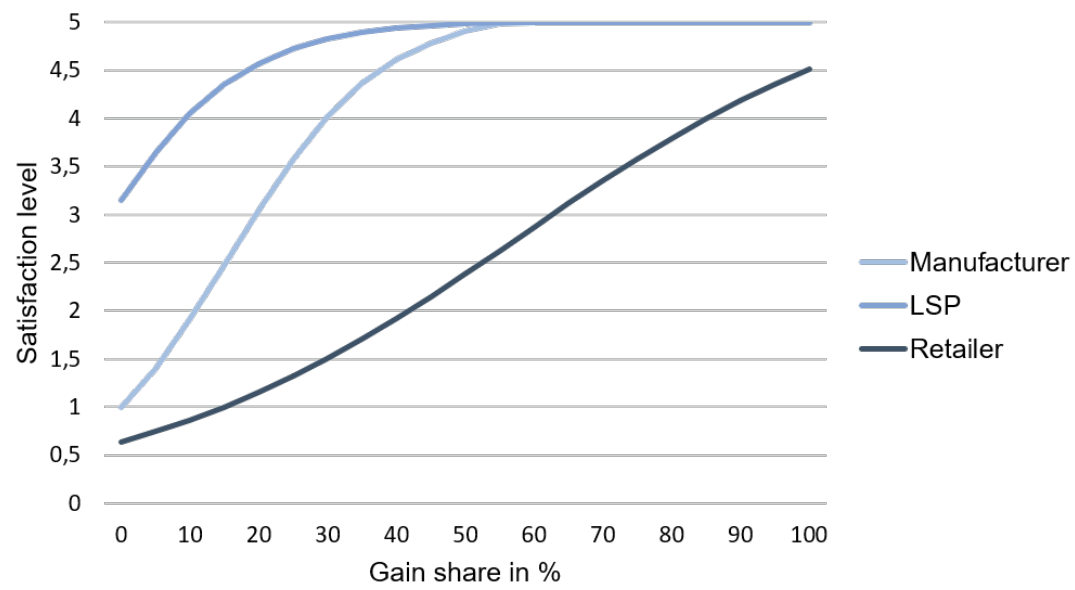

Figure 4.2: Graphical illustration of the satisfaction functions 
In order to perform the gain sharing algorithm, financial information on the SCC is required. Table 4.3 provides an overview of the expected benefits, costs and the resulting profits of the vertical SCC, as already introduced in Chapter 3 (Table 3.2). The resulting total profit achieved by the grand coalition is $205,000 €$.

Table 4.3: Expected benefits, costs and resulting profits of the vertical SCC

\begin{tabular}{c|ccc|c}
\hline & Manufacturer & LSP & Retailer & Overall \\
\hline \hline Benefits & $80,000 €$ & $50,000 €$ & $250,000 €$ & $380,000 €$ \\
Costs & $85,000 €$ & $10,000 €$ & $80,000 €$ & $175,000 €$ \\
Profits & $-5,000 €$ & $40,000 €$ & $170,000 €$ & $205,000 €$ \\
\hline
\end{tabular}

As already outlined in Sections 4.2.1 and 4.2.2, not only knowledge on the grand coalition gain, but also on the subcoalition gains is needed. The gain for a coalition between the manufacturer and the LSP is $9,000 €$, whereas a collaboration between the manufacturer and the retailer results in a coalition gain of 99,000€. A gain of $150,000 €$ can be achieved by a coalition between the LSP and the retailer. If the parties are not collaborating with each other, no gain can be achieved by any party.

Based on the input data described above, the gain sharing algorithm (4.1)-(4.3) for the vertical SCC between one manufacturer, one LSP and one retailer from the Dutch FMCG industry can be specified as follows:

$$
\begin{aligned}
& \min _{0 \leq x_{m}, x_{l}, x_{r} \leq 1} \max \\
& \left\{5.12-\frac{1.23}{0.24+e^{-9 x_{m}}} ; 5.02-\frac{8.48}{1.69+e^{-9 x_{l}}} ; 4.49-\frac{0.72}{0.13+e^{-3.5 x_{r}}}\right\} \\
& \text { s.t. } x_{m}+x_{l}+x_{r}=1 \\
& x_{m}+x_{l} \geq 0.04 \\
& x_{m}+x_{r} \geq 0.48 \\
& x_{l}+x_{r} \geq 0.73
\end{aligned}
$$

\subsubsection{Output Discussion}

In Figure 4.3, the gain allocation for the vertical SCC in the Dutch FMCG industry is depicted. With $68.33 \%$, the retailer receives the largest portion of the gain, followed by the manufacturer with $26.83 \%$. The remaining part of $4.84 \%$ is 
assigned to the LSP, which represents only a small portion of the coalition gain.

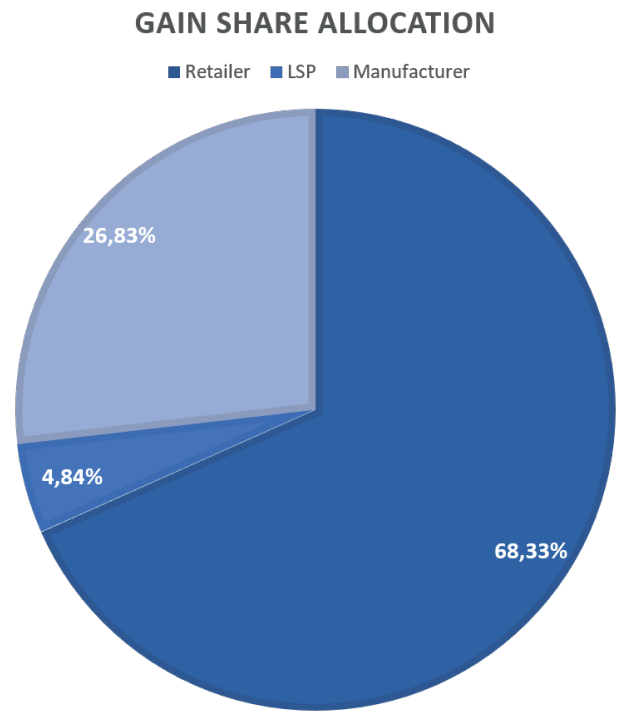

Figure 4.3: Gain share allocation

Table 4.4 presents the satisfaction levels corresponding to the assigned gain shares and regrets. The latter one is calculated by subtracting the actual satisfaction level of the assigned gain share from the maximum possible satisfaction level. The manufacturer's satisfaction level for the assigned gain share of $26.83 \%$ is the largest one with 3.73. The retailer possesses the lowest satisfaction level with 3.25 for the largest gain share. Nevertheless, the retailer has the lowest regret with 1.24. When looking at the regret of the manufacturer and the LSP, it can be seen that the regret is the same with 1.39. When comparing this regret to the retailer's regret, no big difference can be observed, which leads to the conclusion that the optimum is reached.

Table 4.4: Satisfaction levels and regrets of all parties

\begin{tabular}{c|ccc}
\hline & Gain share in \% & Satisfaction level & Regret \\
\hline \hline Manufacturer & 26.83 & 3.73 & 1.39 \\
LSP & 4.84 & 3.63 & 1.39 \\
Retailer & 68.33 & 3.25 & 1.24 \\
\hline
\end{tabular}


The retailer has the most power in the Dutch FMCG supply chain and in addition in this setting the retailer has the highest financial contribution to the coalition gain, which results in low satisfaction levels for all gain shares. In turn, this results in the allocation of the largest portion of the coalition gain to the retailer. As already mentioned, the LSP is influenced by the choice-supportive bias. The influence of this bias results in a high acceptance/satisfaction level for all possible gain shares. Obviously, this leads to the smallest gain share.

In order to proof the advantage of the proposed gain sharing system, it is compared to the two most referred to (and preferred) game theoretic allocation methods, the Shapley value and the Nucleolus (Moulin, 1991). Table 4.5 shows the satisfaction levels and the regrets for these two methods. Here, the manufacturer receives the smallest portion of the gain and, compared to the gain sharing system, the satisfaction level is lower resulting in a higher regret. The LSP receives a larger portion of the gain resulting in a very high satisfaction level and in a small regret. The retailer receives a larger portion of the coalition gain according to the Nucleolus and a lower portion according to the Shapley value. As a whole, the maximum regrets in the two game theoretic methods are much higher than in the proposed gain sharing system. This might result in a decreased probability of a sustainable SCC, which is, however, essential for every party in any supply chain (Jap, 2001).

Table 4.5: Satisfaction levels and regrets for the Shapley value and Nucleolus

\begin{tabular}{c|ccc|ccc}
\hline & \multicolumn{3}{|c|}{ Shapley Value } & \multicolumn{3}{c}{ Nucleolus } \\
& Gain Share & Satisfaction & Re- & Gain Share & Satisfaction \\
in $\%$ & Level & gret & in $\%$ & Re- \\
& 17.72 & 2.78 & 2.34 & 2.11 & 1.15 & 3.97 \\
\hline \hline MA & 30.16 & 4.83 & 0.19 & 26.99 & 4.77 & 0.25 \\
LSP & 51.63 & 2.45 & 2.04 & 70.89 & 3.37 & 1.12 \\
RE & & & & & & \\
\hline
\end{tabular}

\subsection{System Stability}

In this section, the gain sharing system stability is investigated. First, basic fairness properties of allocation methods, which represent interesting KPIs, are introduced and the satisfaction of these properties for the developed gain sharing 
system is investigated. This is followed by a sensitivity analysis of the uncertain parameter of the system, the satisfaction functions.

\subsubsection{Fairness Properties}

Considering the characteristics of an SCC, it is essential that any proposed sharing mechanism is desirable on a collaborative and individual level. In addition, it is important to ensure that the applied sharing technique is perceived by the cooperating parties as reasonable and easy to understand. Accounting for these challenges, a general definition of a fair sharing mechanism is difficult to develop. As such, Table 4.6 provides an overview of the basic fairness properties desirable in the SCC context (Guardiola et al., 2007; Leng and Parlar, 2009; Liu et al., 2010; Verdonck, 2017).

Table 4.6: Allocation properties of gain sharing methods

\begin{tabular}{|c|c|}
\hline Property & Definition \\
\hline Efficiency & $\begin{array}{l}\text { The total coalition gain is shared as the grand coalition } \\
\text { forms: } \sum_{i \in N} x_{i}=v(N)\end{array}$ \\
\hline $\begin{array}{l}\text { Individual } \\
\text { rationality }\end{array}$ & No partner gains less than their stand-alone gain: $x_{i} \geq v(\{i\})$ \\
\hline $\begin{array}{l}\text { Subgroup } \\
\text { rationality }\end{array}$ & $\begin{array}{l}\text { Parties are never better off forming a subgroup by excluding } \\
\text { other parties: } \sum_{i \in S} x_{i} \geq v(S)\end{array}$ \\
\hline Stability & $\begin{array}{l}\text { No single participant or (sub)coalition of participants } \\
\text { of the collaboration would benefit from leaving the } \\
\text { grand coalition: } \sum_{i \in N} x_{i}=v(N) \text { and } \sum_{i \in S} x_{i} \geq v(S)\end{array}$ \\
\hline Additivity & $\begin{array}{l}\text { The profit allocation of a combination of several separate } \\
\text { coalitions is equal to the sum of the separate allocation } \\
\text { values of these coalitions: } x(i \cup j)=x(\{i\})+x(\{j\})\end{array}$ \\
\hline
\end{tabular}

Since the fairness properties of the developed allocation system may have a significant influence on the sustainability of the SCC, the satisfaction of these properties for the proposed gain sharing system were tested by means of an illustrative numerical example. The example relates to the already outlined vertical SCC between the manufacturer (M), LSP (L) and retailer (R) from the Dutch FMCG industry. The third column of Table 4.7 lists the collaborative profits for all possible (sub)coalitions. The second column lists the profits allocated by the 
developed gain sharing algorithm when the grand coalition is formed.

Table 4.7: Collaborative profit and allocated profit for all (sub)coalitions

\begin{tabular}{c|cc}
\hline (Sub)coalition & Allocated Profit & Collaborative Profit \\
\hline \hline M & $54,999.45$ & 0.00 \\
L & $9,930.20$ & 0.00 \\
R & $140,070.35$ & 0.00 \\
ML & $64,929.65$ & $9,000.00$ \\
MR & $195,069.80$ & $99,000.00$ \\
LR & $150,000.55$ & $150,000.00$ \\
MLR & $205,000.00$ & $205,000.00$ \\
\hline
\end{tabular}

Analyzing this example, it can be stated that the proposed gain sharing system is efficient. The total coalition gain is shared as the grand coalition forms $(205,000$ $=205,000)$. Moreover, constraint 4.2 of the gain sharing system satisfies the efficiency property. The proposed gain sharing system also satisfies the individual rationality property. The stand-alone gain for each party is 0 , while the allocated gain for each individual party is larger than 0 . In addition, the subgroup rationality property is satisfied. No subcoalition has the incentive to leave the grand coalition and be better-off when acting alone. This is because the collaborative profit of subcoalitions is smaller than its allocated profit in a grand coalition. Constraints 4.2 and 4.3 guarantee the stability of the allocation defined by the proposed system. Finally, the additivity property is satisfied. The profit allocation of any (sub)coalition is equal to the sum of the separate allocation values of the (sub)coalition members, e.g., for M and L, 64, $929.65=54,999.45+9,930.20$. The analysis indicates the fulfillment of all considered fairness properties. As a result, it can be stated that the proposed gain sharing system can be perceived as fair and will likely result in sustainable SCCs.

\subsubsection{Sensitivity Analysis}

A sensitivity analysis was performed to examine the effect of the uncertain satisfaction functions on the parties' satisfaction levels. Depending on the satisfaction function, the assigned gain to the party will get smaller or larger and the remaining part needs to be split among the other two parties. For example, if the gain share assigned to the manufacturer changes from the current $26.83 \%$ to $20 \%$ of 
the coalition gain, $6.83 \%$ of the collaborative profit will need to be split among the LSP and the retailer. In order to assign the remaining gain share to the LSP and the retailer in a fair way, the gain sharing system was run for an SCC between these two parties. Depending on the outcome of the gain sharing system for $x_{l}$ and $x_{r}$, the remaining part will be split among the two parties. The same holds for the reverse case, namely if the assigned gain share to the manufacturer increases. The results of the gain sharing system for the two-level collaborations are shown in Table 4.8. A collaboration between the manufacturer and the LSP results in an allocated share of $60.82 \%$ to the manufacturer and the rest is assigned to the LSP. The gain share assigned to the manufacturer is halved (29.63\%) if the manufacturer and the retailer are collaborating. The highest gain share is assigned to the retailer (83.32\%) if this party is engaged in a two-level collaboration with the LSP.

Table 4.8: Gain share allocation for two-level collaborations

\begin{tabular}{c|ccc}
\hline Coalition & $x_{m}$ & $x_{l}$ & $x_{r}$ \\
\hline \hline ML & 0.6082 & 0.3918 & \\
MR & 0.2963 & & 0.7037 \\
LR & & 0.1668 & 0.8332 \\
\hline
\end{tabular}

Figures 4.4, 4.5 and 4.6 show the results of the sensitivity analysis for a change in the manufacturer's, the LSP's and the retailer's satisfaction function, respectively. The parties' satisfaction levels vary in relation to changes in the satisfaction functions. A change in the LSP's satisfaction function has the highest impact on the satisfaction levels of the manufacturer and the retailer, see Figure 4.5. If the assigned gain share to the LSP increases, the satisfaction levels of the manufacturer and the retailer decrease rapidly. Both satisfaction functions follow an S-shape and do not cross each other, thus indicating that, in comparison to the manufacturer, the retailer is always less satisfied.

The LSP is the party that is always satisfied no matter what gain share it is assigned. As a result, the satisfaction levels of the LSP are not highly influenced by changes in the retailer's and manufacturer's satisfaction function, as demonstrated in Figures 4.4 and 4.6. 


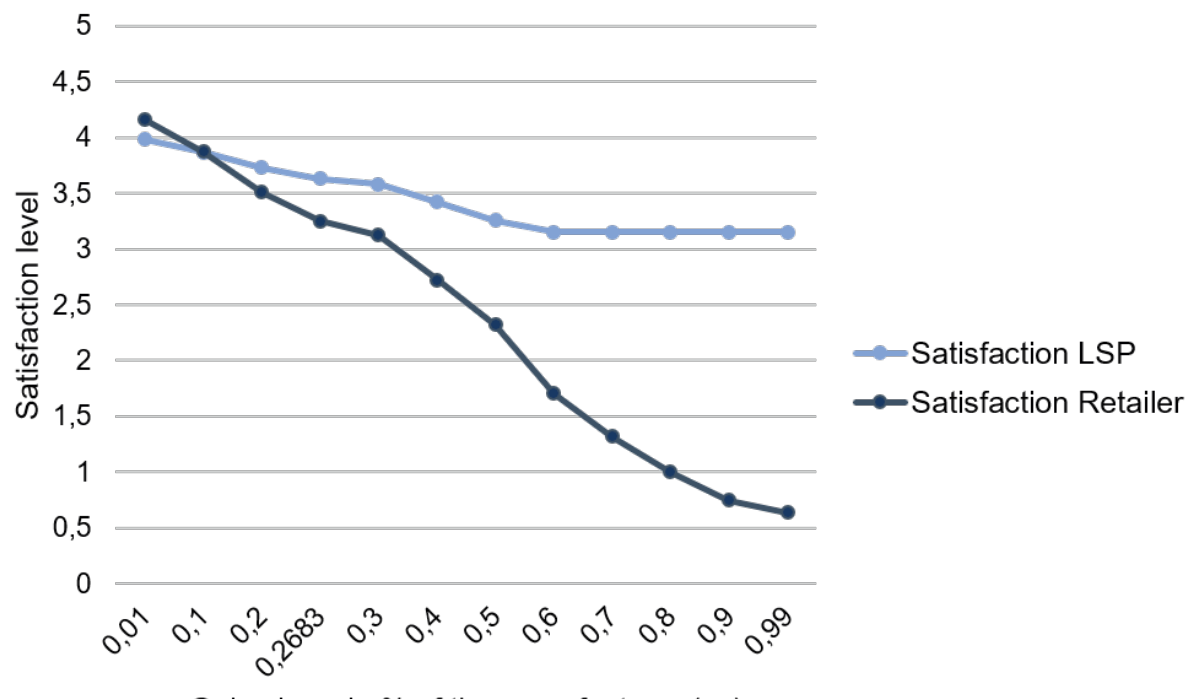

Gain share in $\%$ of the manufacturer $\left(x_{m}\right)$

Figure 4.4: Sensitivity analysis for the manufacturer's satisfaction function

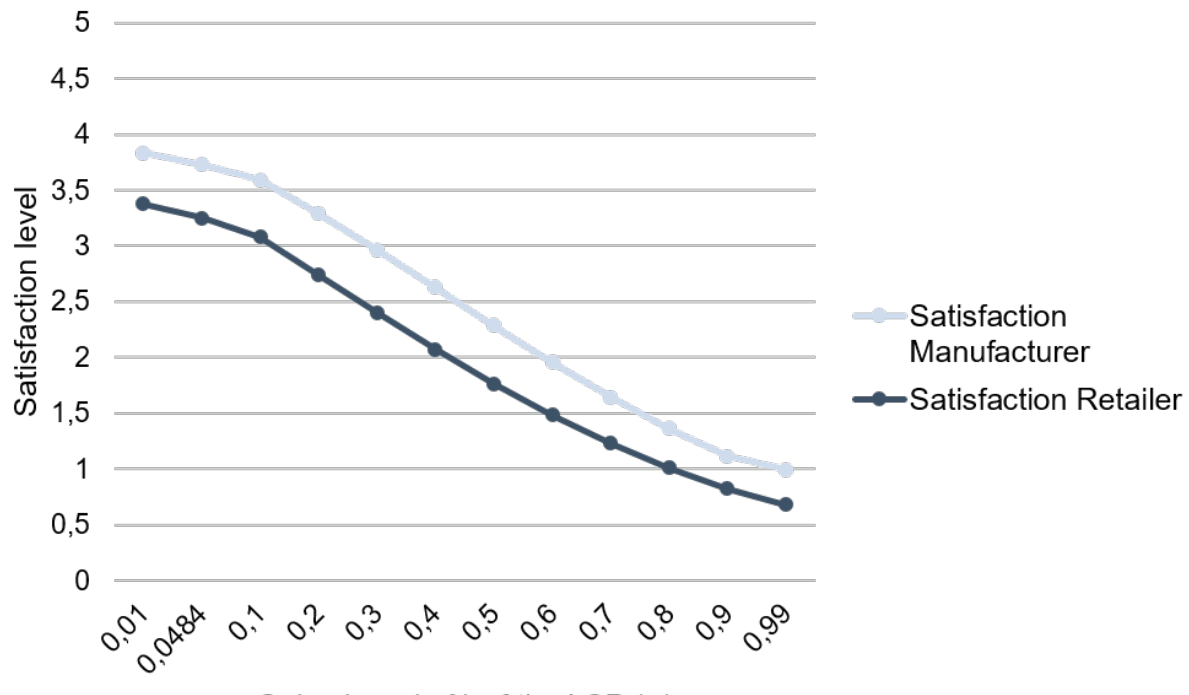

Gain share in \% of the LSP $\left(x_{1}\right)$

Figure 4.5: Sensitivity analysis for the LSP's satisfaction function 


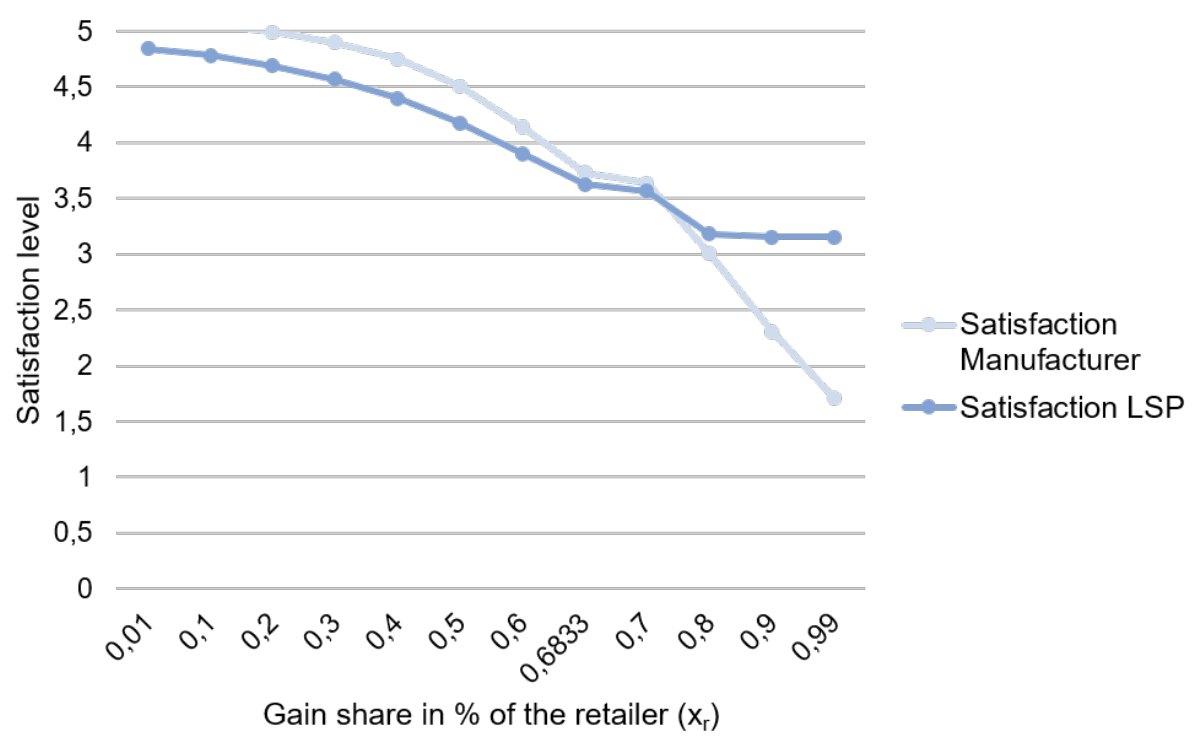

Figure 4.6: Sensitivity analysis for the retailer's satisfaction function

Concluding this section, especially the retailer's and the manufacturer's satisfaction levels are highly influenced by a change in the satisfaction functions. Therefore, in order to assure a stable SCC, a precise determination of the satisfaction functions is important. One essential aspect achieving a precise determination of the satisfaction functions are honest answers in the questionnaires. However, if the survey questions demand responses which are too revealing, participants may refuse to answer or even lie (Clark and Desharnais, 1998; Warner, 1965). Anonymity is one option which might increase the probability of receiving honest answers (Mühlenfeld, 2005). In the survey in Chapter 3, anonymity was guaranteed. Through the use of an online survey, no personal interaction between the interviewer and the interviewee took place. Furthermore, except for one question on the nature of the respondent's supply chain positions, no personal questions were asked. Through the use of self-administered questionnaires and therefore the absence of an interviewer, the probability of getting truthful answers can also be increased (Nederhof, 1985). In addition, according to Mühlenfeld (2005), instructing the participants to answer truthfully before and during the survey might be another way to increase honesty. 


\subsection{Conclusion, Implications and Future Research}

In this chapter, a comprehensive, simple and robust gain sharing system has been introduced. In order to ensure the acceptance and satisfaction of all collaborative parties and to increase the probability of a sustainable SCC, the system focuses on the maximization of the parties' satisfaction by using a minimax regret approach. The proposed gain sharing system has been applied to a vertical SCC in the Dutch FMCG industry to theoretically illustrate the potential and high performance of the system. Results show the maximization of the parties' satisfaction and the decrease of the regrets. In addition, a system stability analysis proved the fairness of the gain allocation and revealed the importance of an accurate determination of the satisfaction functions.

\subsubsection{Theoretical Implications}

The contribution of this chapter to the existing literature is twofold. First, while existing gain sharing methods are not accepted by or satisfactory for the collaborative parties, the scheme developed in this chapter focuses on the maximization of the parties' satisfaction. Second, known game theoretic allocation methods are perceived as too hard to understand and too complex to implement, while the presented method is intuitive and simple.

\subsubsection{Practical Implications}

The new gain sharing system requires only limited input data to provide robust output for the gain sharing decision. In addition, the proposed gain sharing system provides all key characteristics which are appreciated in practice: mathematical simplicity, applicability and transparency. Furthermore, important KPIs such as the optimal gain share allocation and the fairness properties have been introduced in order to support managers to evaluate the performance of the proposed gain sharing system. Moreover, the sensitivity analysis revealed the importance of the precision of the satisfaction functions. In order to achieve this, honest questionnaire replies are essential. 


\subsubsection{Further Research Directions}

This chapter offers several opportunities for further research. In the study, it has been assumed that the only influencing aspect on the parties' satisfaction is the gain share. Further research should include additional influencing aspects such as the amount and the quality of information for parties to share. Furthermore, the proposed gain sharing scheme has been tested in an artificial vertical SCC in the Dutch FMCG industry. Applying the gain sharing system also to horizontal and/or lateral collaborations as well as to a real-life situation may result in stronger support. In addition, the acceptance of the gain sharing system could be observed in practice. The implementation of the new gain sharing system into practice requires a precise determination of the satisfaction functions for each collaborative party individually. Thus, it is of high importance to implement the techniques that ensure and improve honesty in questionnaire replies. 


\section{Chapter 5}

\section{Urban Area Tessellations:}

\section{Are Regular Tessellations}

\section{Good for a Non-uniformly}

\section{Distributed Demand? 1}

\subsection{Introduction}

In the course of the last decades, the population shifted from rural to urban areas; a trend known as urbanization (Cohen, 2006). This is a result of migration due to income growth, but also linked to demographic factors such as a rapid internal urban population growth (Jedwab et al., 2015). In 2018, 55\% of the total population lived in urban regions. The most urbanized continent with $82 \%$ of the population living in urban areas is Northern America, whereas Africa is with $43 \%$ the continent with the lowest amount of people living in urban areas. In Europe, $74 \%$ of the total population is living in metropolises (Statista, 2018). The urban population is expected to grow even further in the next years. In Organisation for Economic Cooperation and Development (OECD) countries such as Austria, Spain and the United States, $83 \%$ of the population is anticipated to dwell in urban regions by 2020 (OECD, 2003) and $54 \%$ of the African population is expected

\footnotetext{
${ }^{1}$ This chapter is based on the paper Grigoriev et al. (2019a), submitted for publication.
} 

Non-Uniformly Distributed Demand?

to live in cities by 2030 (Hay et al., 2005).

Urbanization has a great impact on cities since they are growing both in geographical area and in population, which brings along many societal and logistical challenges (Antrop, 2004; Brinkman, 2016; Cohen, 2006; OECD, 2003). Figure 5.1 shows the shapes of several metropolises all over the world, such as Amsterdam, Beijing, London, Melbourne, Moscow, New York, Paris and Rome. Concentric growth around a historic center is evident in most European or "Old World" cities, like Amsterdam, London, Moscow and Rome. Figure 5.1 shows that these cities are formed around rings and radial lines (depicted on the maps). Typically, demand density for commodities and services is high in the center and decreases towards the suburbs (e.g. Antrop, 2004). This property is very roughly described and there are significant deviations. For instance, Paris can be viewed as a collection of non-concentric rings with many distinct radial lines, see Figure 5.1f. Another example is Beijing, where the map is concentric but instead of rings there are increasing nested rectangles with joints instead of radial lines, see Figure 5.1i.

Following the famous Commissioners' Plan of 1811, see Morris et al. (1811), the shape of Manhattan is a regular rectangular grid, see Figure 5.1g. This pattern was specifically designed and implemented for better accessibility to public services and goods. Many cities in the "New World" took over this practice such as Melbourne depicted in Figure 5.1h. In the grids, streets/avenues are placed horizontally and vertically at an angle of approximately 90 degrees. In grid-shaped cities, the demand density is supposed to be almost uniform: the demand is meant to concentrate along streets/avenues and to decrease towards the block centers, while all blocks are meant to be very similar to each other. However, even in the "New World" cities, the population and the demand are not uniformly distributed. 


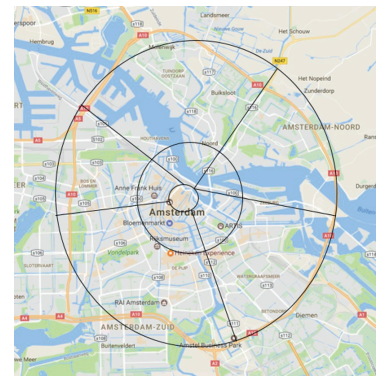

(a) Shape of Amsterdam

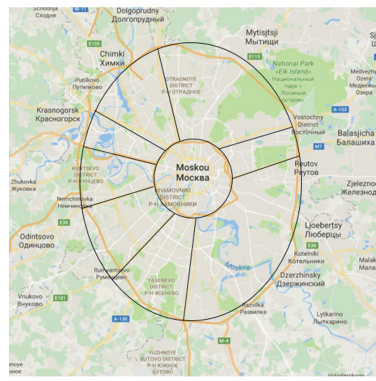

(d) Shape of Moscow

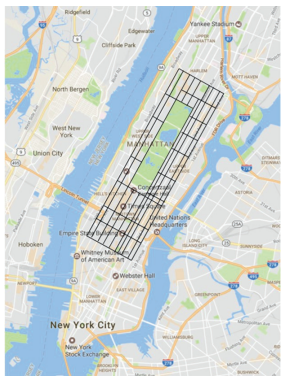

(g) Shape of New York City

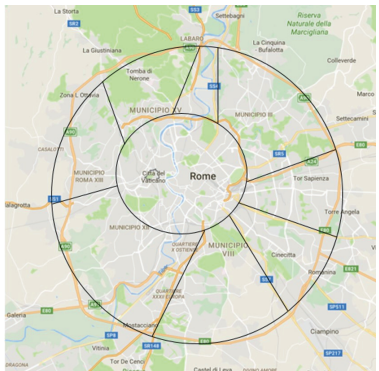

(b) Shape of Rome

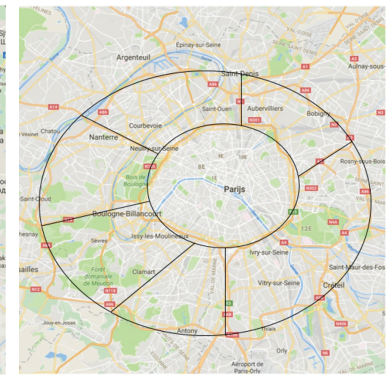

(e) Shape of Paris

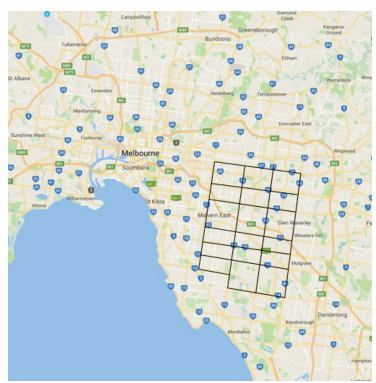

(h) Shape of Melbourne

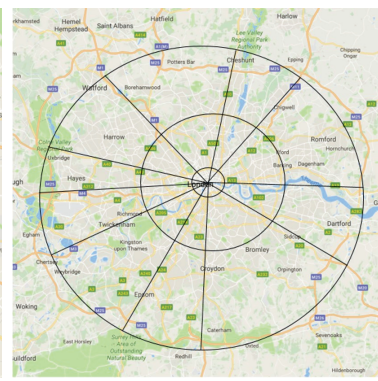

(c) Shape of London

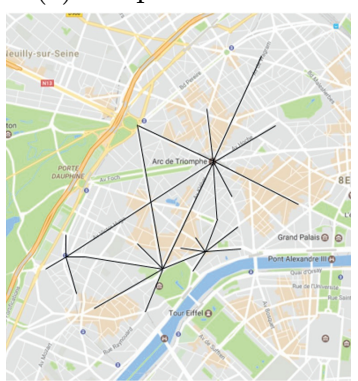

(f) Shape of Paris, close up

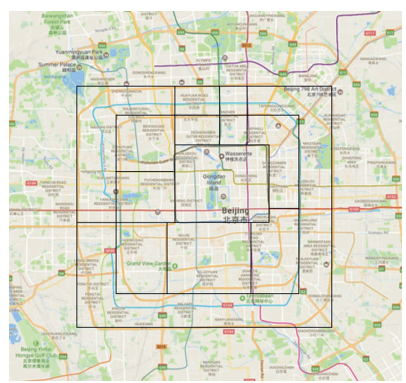

(i) Shape of Beijing

Figure 5.1: Shapes of selected metropolises

In geometry, tessellations (also known as tilings) are used to cover a plane without gaps and overlaps using regular polygons. Applied to urban areas, a single regular polygon forms a district with a facility or a commodity distribution center in the center of the polygon. Nowadays, it is quite common to use regular tessellation to subdivide urban areas into commodity/service distribution districts under the assumption of a uniform demand distribution. However, the population and urban activities are not uniformly distributed (Narula and Ogbu, 1979). To the best of the author's knowledge, until now no research has focused on the perfor- 
mance of regular tessellations under a non-uniform demand distribution. Hence, the central idea of the present chapter is to identify the performance of regular tessellations under irregularly distributed demand. Next to the regular tessellation, the performance of semi- as well as demi-regular tessellations is evaluated. In addition, it will be investigated if there is one (semi-, demi-) regular tessellation which performs best under a given city layout and commodity demand distribution.

As the key performance measure of a tessellation, the objective value of the well-known facility location problem is used since facility location problems are omnipresent in location theory, see e.g. Current et al. (1990). The goal of the facility location problem is to find locations for facilities (commodity distribution centers) that minimize the total costs, including transport as well as facility fixed costs. In this chapter, the objective values for different facility location patterns resulting from the different investigated tessellations are compared in terms of the total costs under several non-uniform demand distributions that are common in practice. This is explained in detail in Section 5.3.2.

The remainder of the chapter is organized as follows. In Section 5.2, basic terminology and contemporary literature on tessellations is briefly outlined. Next, the research methodology is depicted in Section 5.3. In Section 5.4, the performance of (semi-, demi-) regular tessellations as solutions to the facility location problem is studied under given city layouts and demand distributions. In addition, two new tessellations are introduced and analyzed. Finally, concluding remarks will be given in Section 5.5.

\subsection{Tessellations}

A tessellation is a technique to cover a plane with shapes without gaps or overlaps. Whenever a tessellation is made up of congruent regular polygons, it is referred to as a regular tessellation (Grunbaum and Shephard, 1977). There are only three regular polygons that can cover an entire plane: triangle, square and hexagon. Figure 5.2 shows examples for planes tessellated with these regular polygons (Mallozzi et al., 2017). 

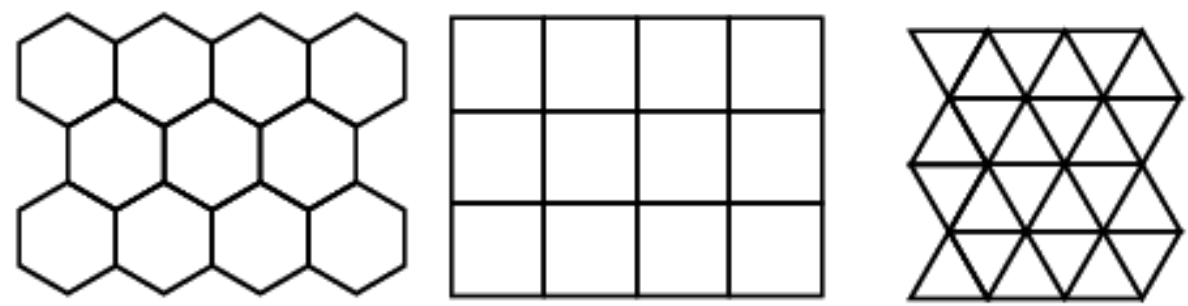

Figure 5.2: Regular tessellations

Whenever a variety of regular polygons are used to cover an area, it is referred to as a semi-regular tessellation. These tessellations have an important property: the arrangement of polygons at every vertex is identical (Watson, 1973). Unlike the regular and semi-regular tessellations, demi-regular tessellations allow for two or more types of regular polygon arrangements at a vertex (Yan et al., 2017). In total, there are eight semi-regular tessellations (Hann, 2013) and 14 demi-regular tessellations (Critchlow and Critchlow, 1969). In Figures 5.3a and 5.3b, examples for the semi-regular and demi-regular tessellations are given, respectively.

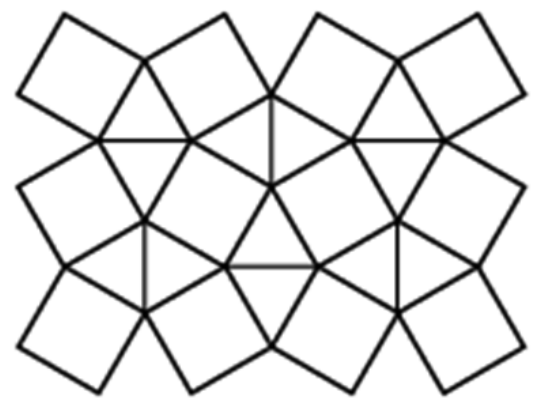

(a) Semi-regular

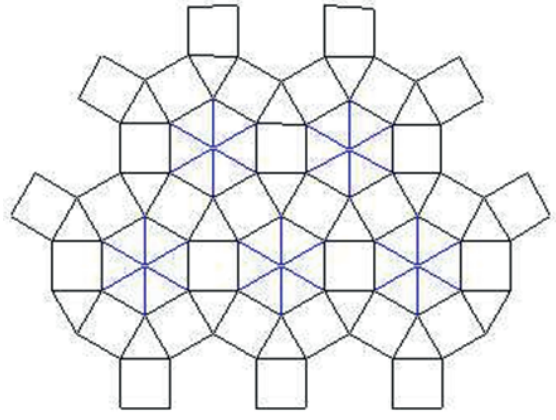

(b) Demi-regular

Figure 5.3: Semi- and demi-regular tessellations

From all introduced tessellations, the hexagonal tessellation is assumed to be most fitting for the description of a uniformly distributed population. The mathematical reason for the preference of the regular hexagon for practical applications is that this shape can cover the plane with the smallest number of tiles (Mallozzi et al., 2017; Schultz, 1970), which is the most efficient economic solution under regularly distributed demand. Hexagonal tessellations have been studied in various 
settings and implemented in many practical applications under the assumption of a regular demand distribution, e.g., in wireless sensor networks (Liu et al., 2007; Patel and Joshi, 2009), and even in the areas of science quite distant from location theory, like image processing and parallel computing (Stojmenovic, 1997). As was mentioned in the introduction, under a non-uniformly distributed demand regular tessellations have hardly been studied so far. In the present literature, only irregular tessellations such as Voronoi diagrams, also known as Dirichlet tessellations, were applied to problems under the assumption of a non-uniform demand distribution (e.g. Didandeh et al., 2013; Gastner and Newman, 2006; Mendes and Themido, 2004). Irregular tessellations, though economically optimal, are quite impractical as they require detailed prior knowledge of the (future) demand distribution, which is barely possible in urban planning (Davis, 1965). Given the impracticality of irregular tessellations, in this chapter the focus is on regular tilings, which are much easier to implement and to use under a non-uniformly distributed demand.

\subsection{Methodology}

\subsubsection{City Layouts and Demand Distribution Models}

In this section two typical city layouts, London representing a concentric and New York City representing a grid structured urban area are outlined. For completeness, the triangular layout, which is quite rare in practice (see Figure 5.1) but methodologically interesting as it represents yet another demand distribution pattern that is close to the uniform demand distribution, is also considered.

Triangular layout In Figure 5.4, the triangular customer spread is shown. Here, a customer is located in every vertex (indicated by a blue cross). Please note that for simplicity, the customers in this study are solely located in the vertexes and not on the edges. As mentioned above, the triangular demand distribution is not common in practice, but it is chosen in order to compare the tessellation performance under an almost uniformly distributed demand with the one under a non-uniformly distributed demand. In this research, two customers are referred to as neighbors if they share one basic tile/triangle. This layout represents a regular 
customer distribution as all distances between neighbors are equal. In this sense, the triangular customer spread is the closest one to a uniform demand distribution. In contrast, consider a grid customer spread: Customers are located in the corner points of square blocks. For each basic tile/square, there are four neighbors but the distances between the neighbors are different: the distance between the diagonal neighbors is factor $\sqrt{2}$ higher than the distances between the neighbors on rectilinear lines. The triangular layout is easy to implement since the customers' allocation on a plane is completely determined by a single parameter, the inter-neighbor distance. In this study, the inter-neighbor distance is chosen to be 1. The customers are located in a square area of $10 \times 10$.

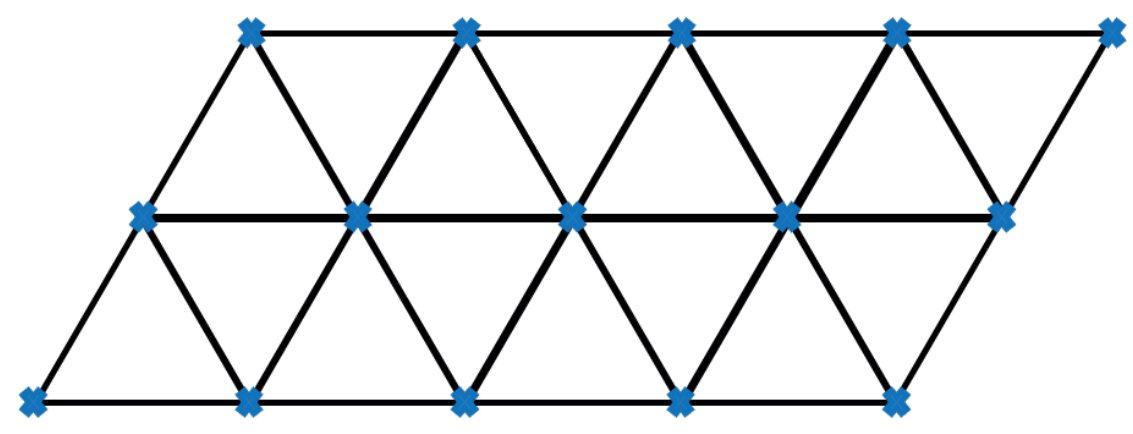

Figure 5.4: Triangular layout

New York City As illustrated in Figures 5.1g and 5.1h, for some urban areas a grid network of roads can be clearly identified. These roads serve as lines of concentrated communication, supply and, therefore, demand distribution. The typical practical setting, and therefore the setting in the present computational study, is that the customers are allocated on the rectilinear grid defining lines equidistant from each other. This layout is also easy to implement. The customers' allocation is determined, again, by a single parameter: the number of customers on a side of a basic tile/square or simply the distance between the nearest neighbors. In the New York City layout, the $10 \times 10$ square grid with a tile length of 1 represents the city map. Figure 5.5 shows an example of the New York City layout with a population of five customers per edge, indicated by the crosses. 
5 Urban Area Tessellations: Are Regular Tessellations Good for a Non-Uniformly Distributed Demand?

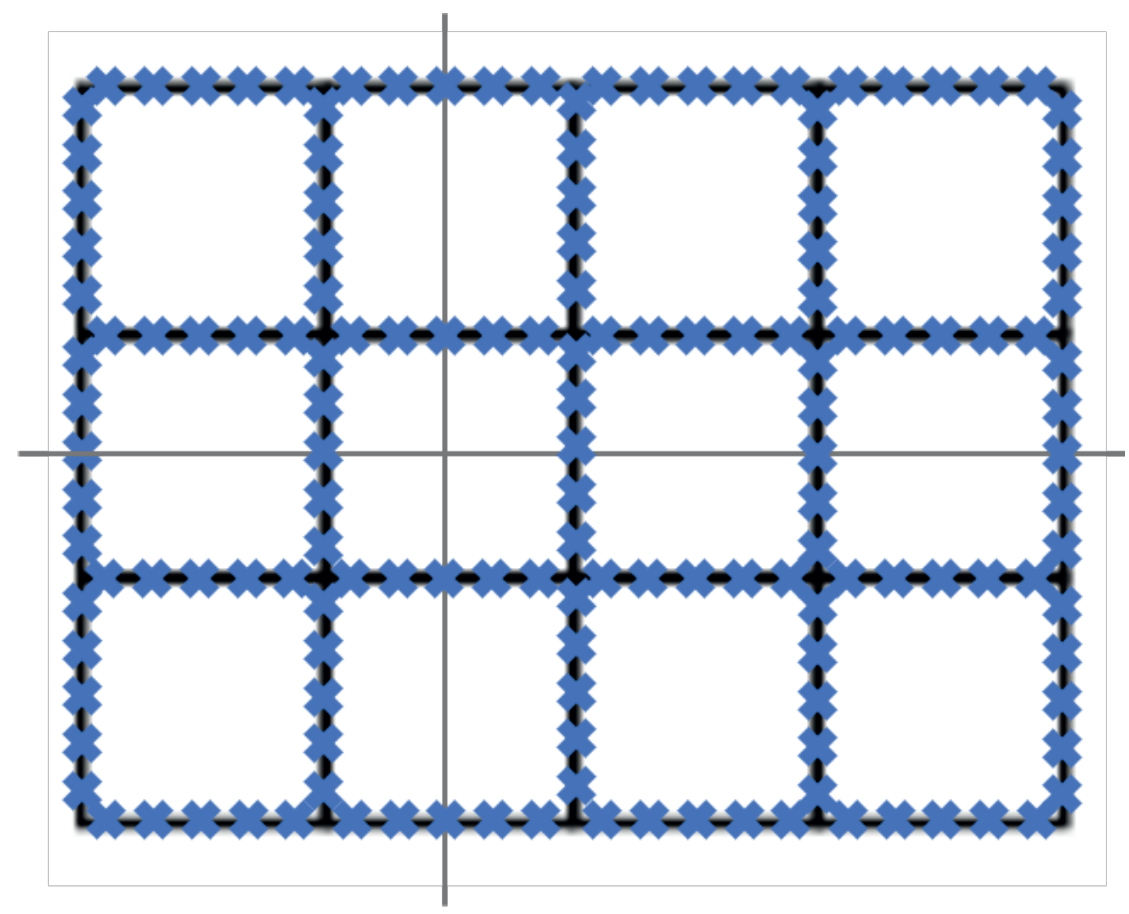

Figure 5.5: New York City layout

London Apart from the grid-shaped cities, especially cities in the "Old World" exhibit a shape of circles with radii-lines, see Figures 5.1a to 5.1d. Since this leads to a completely different demand distribution, another customer spread is introduced. Figure 5.6 shows an example of this customer spread called London. Around the center, there are two (or more) circles of different radii. On each circle and on each radius-line, the customers are evenly distributed. In Figure 5.6, each circle contains 36 customers, yielding that customers are located at a degree of 0 , $10,20,30, \ldots, 350$. Furthermore, on each radius-line at $0,30,60,90, \ldots, 330$ degrees, there are four additional customers: two between the origin and the inner circle and two between the inner and the outer circle. Therefore, on each radius-line there are 6 customers. Note that such a setting represents a city layout where the customers' density around the origin, the city center, is higher than the customer density in the suburbans. 


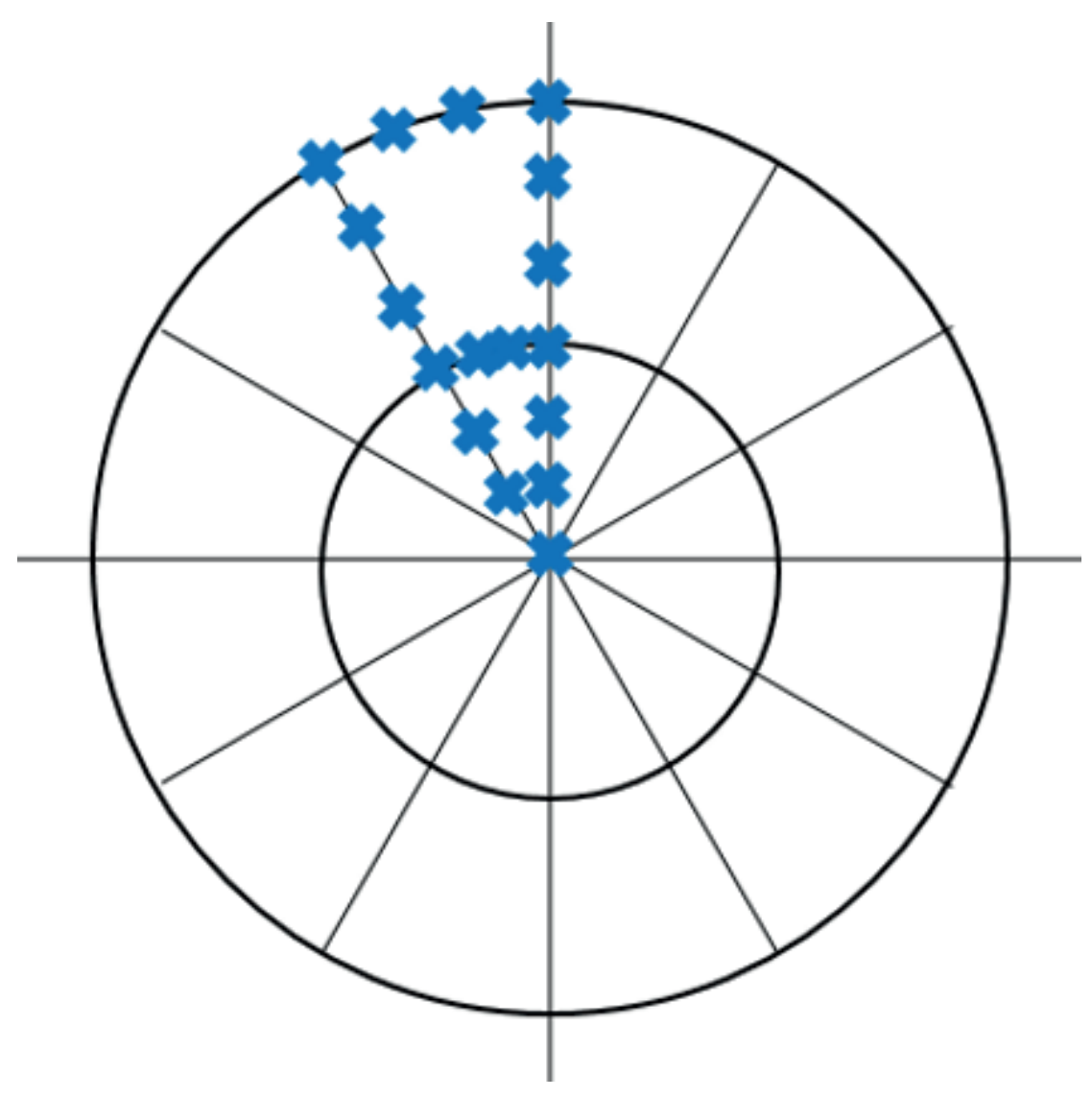

Figure 5.6: London layout

\subsubsection{Suitable Tessellations}

A computational study was conducted to test several tessellations and measure their performance on different layouts. Among all known regular, semi-regular and demi-regular tessellations, the ones depicted in Figure 5.7 were chosen. For clarification, the dots in the polygons in Figure 5.7 represent locations of facilities. The dots are exactly in the center of every tile. The possible location of the facilities depends therefore on the chosen tessellation. Note that for every (semi-, demi-) regular tessellation, the side length of any tile is the same. Let this parameter - the side length of a tile - be denoted by $l$. Given $l$, every (semi-, demi-) regular tessellation is completely determined. The larger/smaller the value of $l$ the fewer/more tiles are needed to cover the area, respectively. Since each tile 
contains a single facility in the center, the amount of facilities decreases/increases in $l$, respectively.

The choice for a (semi-, demi-) regular tessellation can be explained as follows. The choice was based on the range of the tile areas and the regularity of the facility distribution. Note that even for the same side length $l$, the areas of different tiles are the same (Winkler et al., 2015). Tessellations with a high discrepancy of tile areas are seen as impractical as they lead to significant differences in facility capacities. In addition, some semi- and demi-regular tessellations exhibit irregular patterns of the facilities distribution: there are points/areas/stripes of high or low concentration of facility. This asymmetry is not appreciated in practice.

Wolfram (2017) displays all possible semi- and demi-regular tessellations. A reader can verify that the five selected tessellations are, indeed, the most balanced ones with respect to the two criteria outlined above.

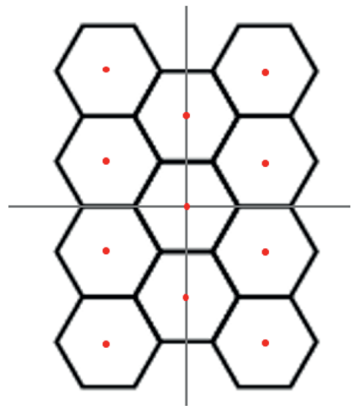

(a) Hexagon tessellation

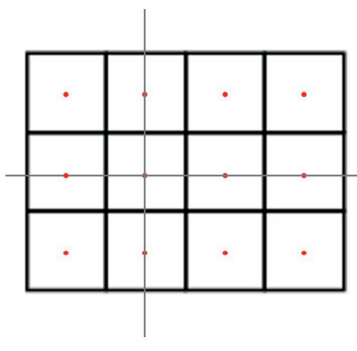

(b) Square tessellation

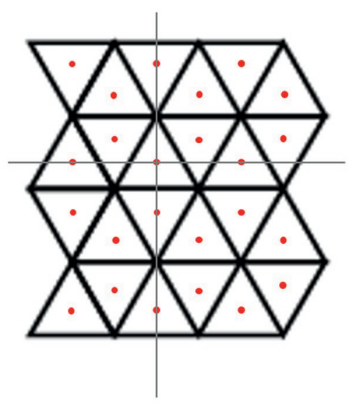

(c) Triangle tessellation
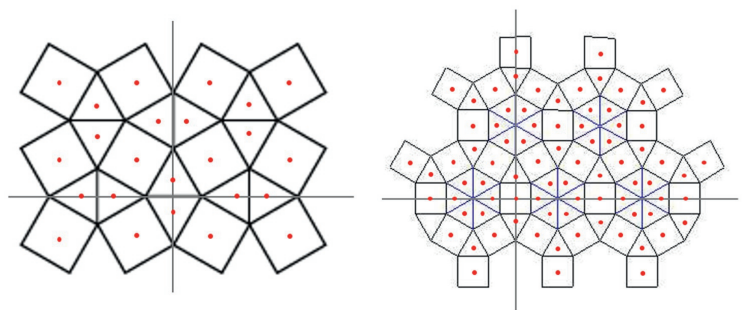

(d) Semi-regular tessellation

(e) Demi-regular tessellation

Figure 5.7: Tessellations used in research 


\subsubsection{Performance of Tessellations on Different Layouts}

To analyze the tessellation performance on the three demand distributions, the tessellation-determined layer of facilities and the layout-determined layer of customers are put on top of each other. The performance of the tessellations is compared in terms of costs. As mentioned in the introduction of this chapter, the facility location objective is considered for this purpose. The mathematical programming formulation for the facility location problem can be described as follows. Let $C$ be the sets of customers, as described in Section 5.3, and $F$ be the sets of potential facilities, as described in Section 5.3.1. Furthermore, let $d_{i j} \in C, j \in F$, denote the distance between customer $i$ and facility $j$ and let $c_{j}, j \in F$, be the fixed cost to open/operate facility $j$. The facility location problem has two binary variables. Let $y_{j}, j \in F$ take value 1 if facility $j$ is open and the value 0 otherwise. Let $x_{i j}, i \in C, j \in F$ take the value 1 if customer $i$ is served at facility $j$ and the value 0 otherwise. Then, the integer linear program modeling the facility location problem reads:

$$
\min _{x, y} \sum_{i \in C} \sum_{j \in F} d_{i j} x_{i j}+\sum_{j \in F} c_{j} y_{j}
$$

subject to

$$
\begin{aligned}
\sum_{j \in F} x_{i j}=1, & \forall i \in C ; \\
x_{i j} \leq y_{j}, & \forall i \in C, j \in F ; \\
x_{i j}, y_{j} \in\{0,1\}, & \forall i \in C, j \in F .
\end{aligned}
$$

For the computational experiments, it is assumed that (a) the distances between the facilities and the customers are measured using the Euclidean distance omitting obstacles; (b) all facilities are identical with respect to fixed costs; (c) for all customers, per-unit-distance transport costs are the same. The author is aware that these are simplistic assumptions deviating from reality, where fixed and transport cost structures are quiet diverse. However, such simplicity provides a deeper insight and intuition than any study based on very diverse urban network cases. It is further implicitly assumed that all facilities are homogeneous, i.e. all facilities provide identical services, have identical prices, opening hours and the 
same quality of goods/services. In addition, unlimited supply of goods/services at any facility and an unlimited unit demand of customers are assumed. As a result of these implicit assumptions, it may be claimed that each customer receives services at the closest facility.

In the experiments, the tessellation performance is studied under varying ratios between facility fixed costs and per-unit-distance transport costs. Four different cost structures are used: 1:1, 1:10, 1:50, 1:100. Here, in contrast to the literature on a classic facility location problem, more weight is put on the transport costs than on the fixed costs. This is a valid assumption for transport and telecommunication industries as well as for the water supply. For some other industries, e.g. financial services and retail including food supply, the fixed costs should receive greater weight than the transport costs.

The setup for the computational experiments is as follows. For each of the three city layouts, for each of the five selected tessellations, and for each of the four cost structures, the optimal length $l$ of the tile side is determined using an approximate solution to the facility location problem, in which all facilities are open. Note that the tile side also influences the number of facilities. In addition to the optimal tile length $l$, the transport costs (Transp) - defined as $\sum_{i \in C} \sum_{j \in F} d_{i j} x_{i j}$-, the fixed costs (Fixed) - defined as $\sum_{j \in F} c_{j} y_{j}$ - and the total costs (Total) - defined as $\sum_{i \in C} \sum_{i \in F} d_{i j} x_{i j}+\sum_{j \in F} c_{j} y_{j}$ - are reported as the solution to the facility location problem. The value for the tile length is found by means of an exhaustive search in a range of $l \in[0.1,3]$, discretized to one decimal. Solutions to the facility location problem are obtained by computing the nearest tessellation-determined facility for each customer and calculating the corresponding transport costs.

\subsection{Tessellation Comparison through Computa- tional Experiments}

\subsubsection{Triangular City Layout}

As already outlined, the triangular city layout is mapped on an area of $10 \times 10$. The same area is used for the tessellations. In Tables 5.1 and 5.2, optimal values for the side length of each polygon $l$ and the costs in optimal solutions are reported for each of the selected tessellations and cost structures (ratios). 
In case of a 1:1 ratio of opening vs. transport costs, the square tessellation yields the lowest total cost of 101.97 at a tile side length of $l=2$ and 25 open facilities. In contrast, the tessellation which exhibits the weakest performance is the triangular tessellation. It uses 49 open facilities. Although this high number of facilities reduces the transport costs, the gain in transport does not compensate for the high opening cost. This is a first striking result indicating there is no perfect match between the tessellation shape and the demand distribution shape.

Even though the square tessellation was the tessellation exhibiting the lowest cost for a 1:1 cost ratio, for all remaining cost structures this tessellation is sub-optimal. The dominant tessellation for the remaining cost structures is the triangular tessellation. This result indicates that for a demand distribution that is approximating a uniform distribution, the triangular tessellation would be the best choice on average. Note that this is not in line with the suggestions from literature, which propose that the best tessellation is hexagonal in case of a uniformly distributed demand. However, the author is aware that a triangular tessellation is merely approximating a uniformly distributed population and that customers are not randomly distributed across the plane. This needs to be taken into consideration when comparing the computational experiments with the present literature. In addition, the weighted distribution between transport and opening costs should be taken into account. For instance, for the majority of the literature focusing on location addresses cases of medical facility locations. The opening/fixed/operating costs of such a facility are so high that only a few facilities will serve a city. In this case the area coverage argument fits perfectly, which makes the hexagonal tessellation the best choice. However, when the transport costs start dominating the fixed costs it is rather beneficial to open several extra facilities to decrease the transport expenses. Thus, generally, the coverage argument should not be the only driver in decision-making, and the hexagonal tessellation is not necessarily the best layout for a commodity distribution even under (almost) uniform demand. This is a second interesting finding of these very simple computational experiments. 
5 Urban Area Tessellations: Are Regular Tessellations Good for a Non-Uniformly Distributed Demand?

\begin{tabular}{l|cccc|cccc}
\hline & \multicolumn{5}{|c|}{$1: 1$ Cost Ratio } & \multicolumn{4}{c}{$1: 10$ Cost Ratio } \\
& $l$ & Transp & Fixed & Total & $l$ & Transp & Fixed & Total \\
\hline \hline Triangular & 2.2 & 61.79 & 49 & 110.79 & 1 & 173.21 & 241 & $\mathbf{4 1 4 . 2 1}$ \\
Square & 2 & 76.97 & 25 & $\mathbf{1 0 1 . 9 7}$ & 1.2 & 487.85 & 81 & 568.85 \\
Hexagonal & 1.2 & 79.98 & 23 & 102.98 & 0.6 & 381.41 & 105 & 486.41 \\
Semi-regular & 2.6 & 85.29 & 22 & 107.29 & 1 & 319.97 & 162 & 481.97 \\
Demi-regular & 2.2 & 76.08 & 31 & 107.08 & 1.4 & 389.42 & 95 & 484.42 \\
\hline
\end{tabular}

Table 5.1: Results for the triangular city layout

\begin{tabular}{l|cccc|cccc}
\hline & \multicolumn{4}{|c|}{$1: 50$ Cost Ratio } & \multicolumn{4}{c}{$1: 100$ Cost Ratio } \\
& $l$ & Transp & Fixed & Total & $l$ & Transp & Fixed & Total \\
\hline \hline Triangular & 1 & 866.03 & 241 & $\mathbf{1 , 1 0 7 . 0 3}$ & 1 & $1,732.05$ & 241 & $\mathbf{1 , 9 7 3 . 0 5}$ \\
Square & 0.4 & 830.27 & 625 & $1,455.27$ & 0.4 & $1,660.54$ & 625 & $2,285.54$ \\
Hexagonal & 0.2 & 660 & 941 & 1,601 & 0.2 & 1,320 & 941 & 2,261 \\
Semi- & 0.6 & 954.60 & 456 & $1,410.60$ & 0.4 & $1,329.01$ & 1,008 & $2,337.01$ \\
regular & & & & & & & & \\
$\begin{array}{l}\text { Demi- } \\
\text { regular }\end{array}$ & 0.6 & $1,036.61$ & 459 & $1,495.61$ & 0.4 & $1,327.38$ & 997 & $2,324.38$ \\
\hline
\end{tabular}

Table 5.2: Results for the triangular city layout

\section{New York City}

The city map is again situated on a $10 \times 10$ grid. In Tables 5.3 and 5.4, the results for the New York City layout are displayed. For this demand distribution under the 1:1 cost ratio, the hexagonal tessellation reveals the lowest total costs of 748.85 at a tile side length of $l=0.4$. The hexagonal tessellation is also the best for the 1:10 and 1:50 cost ratios. Interestingly, the triangular tessellation, which was one of the best in triangular layout, turns out to be one of the worst in the New York City layout. More precisely, this tessellation is performing worst for the 1:1 and 1:10 ratios. Surprisingly, the triangular tessellation is the best performing tessellation for a 1:100 cost structure. This leads to the conclusion that there are no strong dominants amongst the tessellations and every specific case requires its own analysis and decision making. 


\begin{tabular}{l|cccc|cccc}
\hline & \multicolumn{4}{|c|}{$1: 1$ Cost Ratio } & \multicolumn{4}{c}{$1: 10$ Cost Ratio } \\
& $l$ & Transp & Fixed & Total & $l$ & Transp & Fixed & Total \\
\hline \hline Triangular & 1 & 571.49 & 241 & 812.49 & 0.4 & $2,260.35$ & 1,505 & $3,765.36$ \\
Square & 0.6 & 509.24 & 289 & 798.24 & 0.4 & $3,076.97$ & 625 & $3,701.97$ \\
Hexagonal & 0.4 & 501.85 & 247 & $\mathbf{7 4 8 . 8 5}$ & 0.2 & $2,464.18$ & 941 & $\mathbf{3 , 4 0 5 . 1 8}$ \\
Semi-regular & 0.8 & 505.45 & 262 & 767.45 & 0.4 & $2,538.1$ & 1,008 & $3,546.1$ \\
Demi- & 0.8 & 534.99 & 245 & 779.99 & 0.4 & $2,718.31$ & 997 & $3,715.31$ \\
regular & & & & & & & & \\
\hline
\end{tabular}

Table 5.3: Results for New York City

\begin{tabular}{l|cccc|cccc}
\hline & \multicolumn{4}{|c|}{$1: 50$ Cost Ratio } & \multicolumn{4}{c}{$1: 100$ Cost Ratio } \\
& $l$ & Transp & Fixed & Total & $l$ & Transp & Fixed & Total \\
\hline \hline Triangular & 0.2 & $4,753.11$ & 5,693 & $10,446.11$ & 0.2 & $9,506.21$ & 5,693 & $\mathbf{1 5 , 1 9 9 . 2 1}$ \\
Square & 0.3 & $10,648.6$ & 1,089 & $11,737.6$ & 0.3 & $21,297.2$ & 1,089 & $22,386.2$ \\
Hexagonal & 0.1 & $6,101.09$ & 3,853 & $\mathbf{9 , 9 5 4 . 0 9}$ & 0.1 & $12,202.20$ & 3,853 & $16,055.2$ \\
Semi- & 0.2 & 6,153 & 4,034 & 10,187 & 0.2 & 12,306 & 4,034 & 16,340 \\
regular & & & & & & & & \\
Demi- & 0.2 & $6,303.61$ & 3,907 & $10,210.61$ & 0.2 & $12,607.2$ & 2,907 & $16,514.2$ \\
regular & & & & & & & & \\
\hline
\end{tabular}

Table 5.4: Results for New York City

\section{London}

The results for the London layout are outlined in Tables 5.5 and 5.6. Considering a 1:1 cost ratio, the triangular tessellation exhibits the lowest costs of 102.63 at the tile side length of $l=2.6$. At the tile side length of $l=1$, the demi-regular tessellation reveals overall costs of 519.87 at a 1:10 cost ratio, representing the lowest value. The square tessellation is the tessellation which reveals the lowest costs at a 1:50 as well as a 1:100 cost structure. Therefore, it can be concluded that also for this customer spread no superior tessellation can be determined. 
5 Urban Area Tessellations: Are Regular Tessellations Good for a Non-Uniformly Distributed Demand?

\begin{tabular}{l|cccc|cccc}
\hline & \multicolumn{4}{|c|}{$1: 1$ Cost Ratio } & \multicolumn{4}{c}{$1: 10$ Cost Ratio } \\
& $l$ & Transp & Fixed & Total & $l$ & Transp & Fixed & Total \\
\hline \hline Triangular & 2.6 & 71.63 & 31 & $\mathbf{1 0 2 . 6 3}$ & 1.2 & 370.82 & 161 & 531.82 \\
Square & 1.8 & 82.23 & 25 & 107.23 & 0.9 & 411.9 & 121 & 532.9 \\
Hexagonal & 1.2 & 91.59 & 23 & 114.59 & 0.4 & 276.45 & 247 & 523.45 \\
Semi-regular & 2 & 97.2 & 40 & 137.2 & 1.1 & 507.06 & 134 & 641.06 \\
Demi-regular & 2.2 & 79.28 & 31 & 110.28 & 1 & 362.87 & 157 & $\mathbf{5 1 9 . 8 7}$ \\
\hline
\end{tabular}

Table 5.5: Results for the London layout

\begin{tabular}{l|cccc|cccc}
\hline & \multicolumn{4}{|c|}{$1: 50$ Cost Ratio } & \multicolumn{4}{c}{$1: 100$ Cost Ratio } \\
& $l$ & Transp & Fixed & Total & $l$ & Transp & Fixed & Total \\
\hline \hline Triangular & 0.8 & $1,211.42$ & 361 & $1,572.42$ & 0.5 & $1,566.63$ & 963 & $2,529.63$ \\
Square & 0.4 & 795.41 & 625 & $\mathbf{1 , 4 2 0 . 4 1}$ & 0.4 & $1,590.82$ & 625 & $\mathbf{2 , 2 1 5 . 8 2}$ \\
Hexagonal & 0.2 & 705.21 & 941 & $1,646.21$ & 0.2 & $1,410.41$ & 941 & $2,351.41$ \\
$\begin{array}{l}\text { Semi- } \\
\text { regular }\end{array}$ & 0.6 & $1,387.34$ & 456 & $1,843.34$ & 0.4 & $1,884.42$ & 1,008 & $2,892.42$ \\
$\begin{array}{l}\text { Demi- } \\
\text { regular }\end{array}$ & 0.5 & 952.66 & 633 & $1,585.66$ & 0.4 & $1,539.37$ & 997 & $2,536.37$ \\
\hline
\end{tabular}

Table 5.6: Results for the London layout

It is not surprising that (semi-, demi-) regular tessellations do not perform well on the concentric demand map. A high demand in the center combined with low demand on the periphery creates a cost disbalance across the map. This demand distribution is different from both the New York City or the triangular one with a grid structure and an almost uniformly distributed demand. For this concentric demand distribution, more facilities in the center and fewer facilities on the periphery would solve the problem. However, at least a certain extend of logic and regularity in the tessellation is maintained. In the following, two new hexagonbased tessellations are introduced. These tessellations are referred to as expanding hexagonal tessellations.

In Figure 5.8, two expanding hexagonal tessellation structures are displayed. Note that none of the triangles or rhombuses in these tessellations contain a facility, e.g., all black areas are facility-free. The idea behind the expanding hexagons is that the size of the tiles increases with the distance from the center. As stated 
in Section 5.3.1, these tessellations would reasonably match a typical concentric demand distribution of "Old World" cities. The two expanding hexagonal tessellations are also in line with the literature: according to Gastner and Newman (2006), an increase of the population density should be associated with an increase in facility density.

The common structure of expanding hexagons can be described as follows. In the expanding hexagon structure 1, the tile side length of the outer (second level) hexagons is three times larger than the tile length of the inner hexagon, see Figure 5.8a. If an additional layer of hexagons was added, the hexagons in the new circle would have a tile length of nine times the length of the most inner hexagons and so on. Each new layer is multiplied by three. For the expanding hexagon structure 2, see Figure 5.8b, the hexagon growth factor is two, i.e, the tile side length of the outer hexagons is twice the side length of the inner hexagons and if another layer of hexagons was added, it would be four times the side length of the inner hexagons and so on.

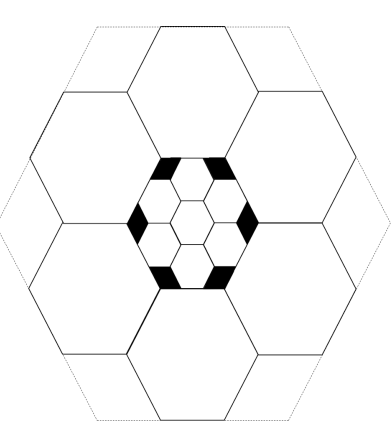

(a) Structure 1: Growth factor 3

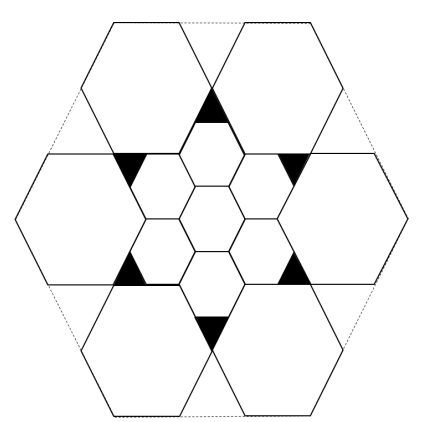

(b) Structure 2: Growth factor 2

Figure 5.8: Expanding hexagons

In the following, the performance of the two expanding hexagonal tessellations is investigated for a concentric customer spread. For the two expanding hexagonal tessellation structures, first a range for the polygon length $l$ is defined in such a way that an additional surrounding layer of even larger hexagons is unnecessary to cover the customer area. This restriction is made in order to match the shape of several "Old World" cities that consist of two circles. Within this range of $l$, the polygon length contributing to the lowest costs is determined. In the case of 
the expanding hexagonal tessellations, the transport costs are the leading costs for determining the best polygon length since due to the restriction to two circles the number of facilities is fixed. Using computational experiments, the performance of the expanding hexagonal tessellations for a 1:1 cost ratio is investigated. Due to the fact that the number of polygons as well as the customer distribution is fixed, changing the cost ratio would not yield any conclusions as to which tessellation is better suited.

The results in Table 5.7 show that for the London layout the expanding hexagonal tessellation with the growth factor 2 reveals the lowest costs. Not surprisingly, in comparison to the (semi-, demi-) regular tessellations, the costs achieved by the expanding hexagonal tessellations are significantly lower. The cost decrease through the use of the expanding hexagonal tessellation structure 2 can be attributed to a reduction of the fixed costs by $58 \%$. This is the result of the length difference of the polygons. Consequently, in peripheral regions facilities can cover a larger area due to the lower population density in these areas.

\begin{tabular}{l|cccc}
\hline & $l$ & Transp & Fixed & Total \\
\hline \hline Structure 1: Growth factor 3 & 0.46 & 70.01 & 13 & 83.01 \\
Structure 2: Growth factor 2 & 0.62 & 62.61 & 13 & 75.61 \\
\hline
\end{tabular}

Table 5.7: New tessellations for London under cost ratio 1:1

In summary, it can be concluded that among regular, semi-regular, and demiregular tessellations there is no clear winner for all cost ratios even in the case of a uniform demand distribution. The message of these computational experiments is that every specific case should be treated individually as the results are relatively difficult to foresee. Furthermore, it can be concluded that in the case of a concentric customer spread, the expanding hexagonal tessellation structure 2 is the recommended tessellation.

\subsection{Conclusion, Implications and Future Research}

In this chapter the performance of (semi-, demi-) regular tessellations under the assumption of a non-uniformly distributed demand has been investigated. Apart from five different "regular" tessellations and three city layouts, four different cost 
structures were considered. The computational study revealed several insights in the performance of (semi, demi-) regular tessellation under an irregular demand distribution.

First, due to the imperfect match between the tessellation and demand distribution shapes, no superior tessellation could be identified. As a result, every specific case should be treated individually. Second, when solving the commodity distribution problem using tessellations, not only the area coverage argument, but also the fixed-to-transport cost ratio is a crucial factor for determining the optimal tiling. When the transport costs dominate the facility fixed costs, the triangular tiling outperforms the hexagonal tessellation even under a uniform demand distribution. Third, under the assumption of a concentric demand distribution, as occurring in "Old World" cities, the concentrically expanding hexagonal tessellations reveal very promising results and outperform all "regular" tessellations. In comparison to the irregular tessellations, which are often introduced for a non-uniformly distributed demand, the expanding hexagonal tessellations do not require detailed (prior) knowledge about the demand distribution and are therefore more practical.

\subsubsection{Theoretical Implications}

This chapter contributes to research by investigating whether (semi-, demi-) regular tessellations can be used for a facility location problem if customers are nonuniformly distributed. Contemporary literature that considered regular tessellation always assumes a uniform demand distribution. Solely irregular tessellations have been applied to problems with a non-uniform demand distribution. Furthermore, two new expanding hexagonal tessellation structures have been developed that outperform (semi-, demi-) regular tessellations for a concentric demand distribution and that are easy to use in practice with limited prior knowledge on demand distribution.

\subsubsection{Practical Implications}

The results of the computational experiments revealed that there is no superior tessellation which can be applied to every demand structure. As a result, each case needs to be treated individually. Fortunately, such experiments are straightforward and easily implementable, even for non-uniform demand distribution. Fixing an 
origin and computing the costs for a range of a single varying parameter $l$ on different tessellations completely determines the choice of the tessellation. Even on a very modest personal computer, this routine does not take much time to compute the best tessellation for the facility location. In addition, a new tessellation has been introduced which can be recommended to be used in case of a concentric demand distribution. The introduced tessellation is very easy to use and to implement in practice due to the fact that limited knowledge on demand distribution is needed in advance.

\subsubsection{Further Research Directions}

This chapter provides several directions for further research. First, it is interesting to know what precisely influences the transport-to-fixed cost ratio in the choice of the tessellation. Therefore, in further research special attention should be paid to the threshold of that cost ratio to determine at which point the triangular tessellation starts outperforming the hexagonal tessellation. Second, in this paper the growth factor two as well as the growth factor three has been taken into consideration for expanding hexagonal tessellations. Further research should investigate the performance of hexagonal tessellations that allow for other growth factors. Third, in this chapter the optimization of costs for a specific tessellation was done by choosing an optimal value of a single parameter, the tile side length $l$. In further research, other parameters, e.g. the selection of an origin and/or rotation of the tiling should be taken into account in order to determine their influence on the tessellation performance. 


\section{Chapter 6}

\section{Final Conclusions and Further Research Directions}

The purpose of this thesis was to study three SCC challenges and to present approaches to overcome these three obstacles to a successful SCC. First, the difficulty of identifying and understanding relevant drivers and resistors for launching a collaboration has been investigated in Chapter 2. Second, the gain sharing challenge has been discussed in-depth in Chapters 3 and 4. Third and last, in Chapter 5 an approach to handle the challenge of finding the right coalition partner based on geographical dimensions has been outlined. This final chapter provides an overall conclusion (Section 6.1) and outlines directions for further research (Section 6.2).

\subsection{Final Conclusions}

SCC has become an important research area ever since increased competition amongst organizations, scarce resources and higher customer expectations forced supply chain partners to engage in collaborations. SCCs offer parties the opportunity to achieve significant competitive advantages, such as cost reduction, time reduction and increased customer satisfaction. However, in practice SCCs often break down due to challenges, such as the identification and understanding of all relevant drivers and resistors for launching the SCC, fair gain sharing and the selection of the right coalition partners. It is thus essential to approach SCCs from a business perspective to find ways to tackle these obstacles. 
The existing literature discussing drivers and resistors for launching an SCC is lacks several important aspects. First, although a significant amount of literature already investigated potential drivers and resistors for launching an SCC, until now a study including all drivers and resistors for launching a collaboration is missing. Existing studies provide insights into a selection of drivers and resistors as well as, in some cases, a selection of perspectives, SCC structures and/or industries. A comprehensive overview of all potential drivers and resistors for all SCC structures, perspectives and/or industries is not provided yet in academic literature. Second, in the literature two types of ambiguity can be identified. The first one is the lack of unique terms for each driver and resistor category. So far, the same term is used for different driver and resistor categories. The second type of ambiguity is that especially drivers are assigned to more than one category. Third, most current work is prone to incompleteness, namely that: drivers or resistors identified in previous research are not presented in more recent literature. Fourth and last, literature identifying drivers and resistors for the FMCG industry is non-existent. The existing literature mainly focuses on forces and barriers. In order to fill these research gaps, a comprehensive, structured and consistent framework including all drivers and resistors for launching SCCs for all collaborative structures, perspectives and industries mentioned in the literature was developed in Chapter 2. The framework consists of four categories: benefits, forces, enablers/barriers and risks. The driver categories, benefits and forces, both include motivating factors for parties to launch a collaboration. Whereas, the benefits represent the intrinsic motivation of a party to launch an SCC, the forces represent the extrinsic motivation. The resistor category, risks, is future-oriented and includes events that might occur in the future but are uncertain yet. The final category, enablers/barriers, combines the driver category, enablers, and the resistor category, barriers. Enablers represents factors which enable someone to collaborate, whereas barriers include factors which keep parties from launching an SCC. Based on an extensive literature review, a connection between these two categories was identified, since for every enabler a corresponding barrier could be observed. To show this connection, the factors of these two categories were combined. When evaluating SCCs, factors will be identified either as an enabler, presence of..., or as a barrier, lack of.... In addition, a case study approach was used to identify relevant drivers and resistors for the Dutch FMCG industry in order to validate the completeness of the conceptual framework. The case study revealed one missing factor in the literature. 
In the Dutch FMCG industry, discussions about gains in the first SCC meeting lead to rough negotiations, which, in some cases, result in a breakdown of the SCC initiative. After adding an additional general factor, which is called presence of neglecting money in the first meeting if it is an enabler and lack of neglecting money in the first meeting if it is a barrier, the framework is complete for all investigated industries, relationships and perspectives. On the practical side, the introduced framework enables parties to more holistically evaluate considered SCCs and thus helps with identifying collaborations with a high failure potential. The evaluation of an SCC is enhanced through an improved understanding and identification of the drivers and resistors for launching SCCs. The understanding is enhanced through a clear distinction between the four driver and resistor categories, consistent definitions and explanations for the influence of these categories on an SCC. Furthermore, the identification is improved through an extensive checklist provided by the framework of all potential drivers and resistors for launching collaborations that need to be taken into account and evaluated on their importance for every potential SCC. Only if the factors that have a positive influence on the collaboration, outweigh those with a negative influence, it is advisable to launch the SCC.

Although other researchers have already outlined the importance of the parties' acceptance of and satisfaction with the assigned gain share for a sustainable SCC, the actual acceptance levels of existing gain sharing methods have not been investigated. Chapter 3 tries to close this research gap through the investigation of the acceptance of selected gain sharing methods (Nucleolus, Shapley value, WCM (power, initiator) and ECM) in vertical SCCs in the Dutch FMCG industry. Furthermore, by investigating the impact of behavioral aspects, namely the availability of information and cognitive biases, on the acceptance decision, Chapter 3 further contributes to the SCM literature by integrating behavioral decision-making research. Up to now, limited research has been published in the logistics and SCM literature dealing with the influence of human behavior, judgment and decisionmaking. However, to ensure the practical validity, the integration of behavioral decision-making is essential since human beings do not think rationally. Rather their decisions are influenced by e.g. limitations in available information as well as cognitive biases. The study in Chapter 3 revealed that in general no gain sharing method is accepted by all collaborative parties. This is due to the fact that the availability of information as well as the cognitive biases have a different influence on each party. Based on these results, one managerial implication is to not 
provide all parties with the same amount of information since this leads to no preferred gain sharing method. For this purpose, it is necessary to observe which and how much information one collaborative party should receive in order to show the highest acceptance level. This information can be obtained by e.g. conducting the survey introduced in Chapter 3. Furthermore, the influence of cognitive biases makes the party's decision unpredictable, since decisions are not made in a rational and consistent way. In order to reduce the occurrence of cognitive biases and simultaneously increase the predictability of the decisions, debiasing-techniques should be applied. One example of a debiasing-technique is to provide all relevant information in an intuitively comprehensive and compelling format. A suggestion is to show a figure which provides information about the gain allocation.

Building upon the research findings of Chapter 3, a comprehensive, simple and robust gain sharing system that is desirable on an individual and collaborative level was proposed in Chapter 4. The developed gain sharing system focuses on the maximization of the parties' satisfaction by using a minimax regret approach in order to overcome the lack of the parties' acceptance of the gain sharing methods. In this way, the newly introduced gain sharing method contributes to an increased probability of sustainable collaborations. Another contribution of the gain sharing system is that it provides managers with a complete gain sharing system and many related useful KPIs to support managers when evaluating the performance of a proposed gain sharing system. In order to theoretically illustrate the potential and performance of the system, it was applied to a vertical SCC between one manufacturer, one LSP and one retailer in the Dutch FMCG industry. The results show a maximization of the parties' satisfaction and a decrease in regrets. In addition, the gain sharing system stability was investigated. The system stability proved the fairness of the gain allocation and revealed the importance of an accurate determination of the satisfaction functions. The proposed gain sharing system can be easily applied in practice since it provides all key characteristics which are appreciated in real-life: mathematical simplicity, applicability and transparency. The system only requires limited input data, namely the parties' satisfaction functions and the financial information of the SCC, to provide a robust output for the gain sharing system. In order to determine the parties' satisfaction functions, the parties' satisfaction levels can be derived through e.g. questionnaires. In this context, it is important to ensure that the questionnaires are answered honestly in order to accurately determine the satisfaction functions. 
There are several techniques to increase the honesty of participants when answering the questionnaire, such as the use of self-administered questionnaires and/or the incorporation of instructions at the beginning and during the surveys asking the respondents to answer the questions truthfully.

Especially for LSPs, geographical coverage is essential but cannot be achieved by one partner alone. However, a horizontal collaboration could provide companies with the opportunity to expand their geographical coverage. In this context, finding the right coalition partner is essential. The research introduced in Chapter 5 focuses on this SCC challenge. For the first time, several known regular, semi-regular and demi-regular tessellations as well as one newly introduced expanding hexagonal tessellation approach were investigated for their performance in locating facilities in a cost-efficient way under the assumption of an irregular demand distribution. The results show that there is no tessellation exhibiting a superior performance for every demand distribution and cost structure. As a result, each specific case needs to be treated individually. However, the research revealed that using tessellations is a good approach to locate facilities and to assign demand to the facilities in a fair and cost efficient manner. In the context of horizontal logistics collaborations, the tessellation approach supports companies in their partner selection process by suggesting where a coalition partner's facilities should be preferably located in order to achieve a significant geographical coverage. Simultaneously, the approach might reduce transport costs in a horizontal collaboration by assigning the demand to the facilities/collaborative parties in a cost efficient manner. In addition, since tessellations cover a plane without overlaps, the competitive pressure that can arise through overlaps in the customer base is avoided.

\subsection{Further Research Directions}

SCC constitutes a broad research domain. Although, in the course of the last years a great amount of literature has been published in this field, several opportunities for further research directions may be identified.

First, this thesis focuses on three challenges for the implementation and success of SCCs. However, as indicated in Section 1.2 there are several more SCC challenges that should be further investigated. Examples are the lack of trust and 
the power imbalance, which are both factors that have a negative impact on the success and sustainability of SCCs.

Second, the majority of research findings are based on data from the Dutch FMCG industry. Further research chould also include other industries and/or geographical areas. Testing the completeness of the framework proposed in Chapter 2 in other geographical areas and/or industries as well as with a greater sample size may result in stronger support. The survey performed in Chapter 3 as well as the gain sharing system proposed in Chapter 4 should also be applied to other geographical areas and/or industries as well as to horizontal and lateral SCCs.

Third, the practical validation of the research work is relevant. The developed framework of Chapter 2 should be applied to real-life cases in order to discover whether the framework actually leads to an increased SCC success. For this purpose, parties that have already participated in a failed collaboration could be interviewed in order to investigate whether the SCC failure would have been prevented if all collaborative parties had taken into account the proposed framework. In addition, the survey outlined in Chapter 3 was conducted in an artificial situation and no incentives, e.g. monetary incentives in order to increase effort and task performance, were provided. Conducting the survey in a real-life situation could identify other important behavioral decision-making aspects. Further research could also apply the gain sharing system proposed in Chapter 4 to real-life SCCs in order to investigate whether it increases the parties' acceptance of and satisfaction with the assigned gain share. The expanding hexagonal tessellation proposed in Chapter 5 could also be applied to a real-life horizontal SCC. In this way, it can be identified whether the partner selection process with regard to the geographical coverage is supported and whether the costs can be reduced.

Finally, the surveys, frameworks, methods and approaches introduced in this thesis focus on certain choices and assumptions. Further research could also take additional aspects into account in order to support the research findings. The focus of the comprehensive framework of all drivers and resistors for launching an SCC is on the relevance of the drivers and resistors for the collaborative parties. However, the importance of the drivers and resistors has not been quantified for a specific partner and collaboration. Quantifying the factors for each partner and collaboration can be beneficial and could also be considered in further research. In 
Chapter 3, the parties' acceptance was investigated for a selection of gain sharing methods. The investigation of the parties' acceptance levels might be extended by observing other gain sharing methods, such as the EPM or WRSM. Moreover, additional weights for the WCM could be investigated. In addition, in the survey the influence of two behavioral aspects, availability of information and cognitive biases, was investigated. Further research could also take into account other aspects. One example would be the availability of time, which is another component of bounded rationality. The lack of available time forces people to use heuristics and cognitive biases. Therefore, it might be interesting to also include the time availability in future surveys. The gain sharing system proposed in Chapter 4 exclusively considers the gain share as an aspect that has an influence on the parties' satisfaction. The research could be extended by including additional influencing aspects such as the amount and quality of information that has to be shared by a party. Furthermore, the tessellation approach introduced in Chapter 5 could consider practical characteristics such as the transport network, different transport modes, heterogeneous facilities as well as geographical barriers for facility locations. 



\section{Bibliography}

Adolfsson, M. and D. Solarz (2005). Power Shift and Retailer Value in the Swedish FMCG Industry. Ekonomiska institutionen.

Ahmad, S. and A. Ullah (2013). Driving forces of collaboration in supply chain: A review. Interdisciplinary Journal of Contemporary Research in Business $\underline{5}(7)$, 39-69.

Akaike, H. (1974). A new look at the statistical model identification. IEEE Transactions on Automatic Control 19(6), 716-723.

Akintoye, A., G. McIntosh, and E. Fitzgerald (2000). A survey of supply chain collaboration and management in the uk construction industry. European Journal of Purchasing \& Supply Management $\underline{6}(3 / 4), 159-168$.

Allison, P. D. (2008). Convergence failures in logistic regression. In SAS Global Forum, Volume 360, pp. 1-11.

Anbanandam, R., D. Banwet, and R. Shankar (2011). Evaluation of supply chain collaboration: a case of apparel retail industry in india. International Journal of Productivity and Performance Management 60(2), 82-98.

Antrop, M. (2004). Landscape change and the urbanization process in europe. Landscape and urban planning $\underline{67}(1), 9-26$.

Attaran, M. and S. Attaran (2007). Collaborative supply chain management: the most promising practice for building efficient and sustainable supply chains.

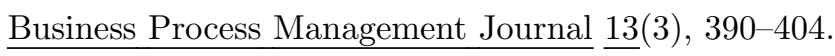


Autry, C. (2011). Collaboration in supply chain: Getting things done beyond the four walls. http://www.scmr.com/article/collaboration_in_the_supply_ chain_getting_things_done_beyond_the_four_walls/. Accessed: 2016-0620.

Barratt, M. (2004). Understanding the meaning of collaboration in the supply chain. Supply Chain Management: An International Journal 9

Baumeister, R. F., E. Bratslavsky, C. Finkenauer, and K. D. Vohs (2001). Bad is stronger than good. Review of general psychology $\underline{5}$ (4), 323-370.

Beach, R., M. Webster, and K. M. Campbell (2005). An evaluation of partnership development in the construction industry. International Journal of Project Management 23(8), 611-621.

Bititci, U., T. Turner, D. Mackay, D. Kearney, J. Parung, and D. Walters (2007). Managing synergy in collaborative enterprises. Production Planning and Control 18 16$), 454-465$.

Bleeke, J. and D. Ernst (1995). Is your strategic alliance really a sale? Harvard Business Review $\underline{73}(1), 97-105$.

Boddy, D., D. Macbeth, and B. Wagner (2000). Implementing collaboration between organizations: an empirical study of supply chain partnering. Journal of Management Studies 37(7), 1003-1018.

Bretherton, P. and P. Carswell (2002). Trust me — i'm a marketing academic. In A cross-disciplinary look at trust, Presentation at the Academy of Marketing Annual Conference, Nottingham University Business School, Nottingham, England.

Brinkman, J. C. (2016). Congestion, agglomeration, and the structure of cities. Journal of Urban Economics 94, 13-31.

Brouthers, K. D., L. E. Brouthers, and T. J. Wilkinson (1995). Strategic alliances: Choose your partners. Long Range Planning 28(3), 18-25.

Bucklin, L. P. and S. Sengupta (1993). Organizing successful co-marketing alliances. The Journal of Marketing $\underline{57}(2), 32-46$. 
Cao, M. and Q. Zhang (2011). Supply chain collaboration: Impact on collaborative advantage and firm performance. Journal of Operations Management $29(3)$, 163-180.

Clark, S. J. and R. A. Desharnais (1998). Honest answers to embarrassing questions: Detecting cheating in the randomized response model. $\underline{\text { Psychological }}$ Methods $\underline{3}(2), 160-168$.

Cohen, B. (2006). Urbanization in developing countries: Current trends, future projections, and key challenges for sustainability. Technology in Society $28(1-2)$, 63-80.

Commission, E. (2012). "horizontal cooperation agreements". http://ec. europa.eu/competition/antitrust/legislation/horizontal.html. Accessed: 2017-03-02.

Cooper, M. C., D. M. Lambert, and J. D. Pagh (1997). Supply chain management: more than a new name for logistics. The International Journal of Logistics $\underline{\text { Management }} \underline{8}(1), 1-14$.

Critchlow, K. and K. Critchlow (1969). Order in space: a design source book. Thames \& Hudson,.

Cruijssen, F. (2006). Horizontal cooperation in transport and logistics. CentER, Tilburg University.

Cruijssen, F. (2012). Collaboration concepts for co-modality' $\left(\mathrm{co}^{3}\right)$.

Cruijssen, F., M. Cools, and W. Dullaert (2007a). Horizontal cooperation in logistics: opportunities and impediments. Transportation Research Part E: Logistics and Transportation Review $43(2), 129-142$.

Cruijssen, F., W. Dullaert, and H. Fleuren (2007b). Horizontal cooperation in transport and logistics: a literature review. Transportation Journal 46(3), 22 39.

Current, J., H. Min, and D. Schilling (1990). Multiobjective analysis of facility location decisions. European Journal of Operational Research 49(3), 295-307.

Daugherty, P. J., R. G. Richey, A. S. Roath, S. Min, H. Chen, A. D. Arndt, and S. E. Genchev (2006). Is collaboration paying off for firms? Business Horizons $\underline{49}(1), 61-70$. 
Davis, K. (1965). The urbanization of the human population. Scientific American 213(3), 40-53.

de Kok, T., J. Dalen, and J. Hillegersberg (2015). Cross-chain collaboration in the fast moving consumer goods supply chain. University of Eindhoven.

de Leeuw, S. and J. Fransoo (2009). Drivers of close supply chain collaboration: one size fits all? International Journal of Operations \& Production Management 29(7), 720-739.

De Martino, B., D. Kumaran, B. Seymour, and R. J. Dolan (2006). Frames, biases, and rational decision-making in the human brain. Science 313(5787), 684-687.

Didandeh, A., B. S. Bigham, M. Khosravian, and F. B. Moghaddam (2013). Using voronoi diagrams to solve a hybrid facility location problem with attentive facilities. Information Sciences 234, 203-216.

Eisenhardt, K. M. (1989). Building theories from case study research. Academy of Management Review $\underline{14}(4), 532-550$.

Ellram, L. M. and M. C. Cooper (1990). Supply chain management, partnership, and the shipper-third party relationship. The International Journal of Logistics Management $\underline{1}(2), 1-10$.

Evans, J. (2012). Managing assumptions, risks and impediments in strategic planning. http://blog.vistage.com/business-strategy-and-management/ managing-assumptions-risks-and-impediments-in-strategic-planning/. Accessed: 2016-01-10.

Evans, J. R. and A. Mathur (2005). The value of online surveys. Internet $\underline{\text { Research }} \underline{15}(2), 195-219$.

Fang, S.-R., Y.-S. Chang, and Y.-C. Peng (2011). Dark side of relationships: A tensions-based view. Industrial Marketing Management 40(5), 774-784.

Fawcett, S. E., S. L. Jones, and A. M. Fawcett (2012). Supply chain trust: The catalyst for collaborative innovation. Business Horizons 55(2), 163-178.

Fawcett, S. E. and G. M. Magnan (2002). The rhetoric and reality of supply chain integration. International Journal of Physical Distribution \& Logistics Management $32(5), 339-361$. 
Fawcett, S. E., G. M. Magnan, and M. W. McCarter (2008a). Benefits, barriers, and bridges to effective supply chain management. Supply Chain Management: $\underline{\text { An International Journal }} \underline{13}$ (1), 35-48.

Fawcett, S. E., G. M. Magnan, and M. W. McCarter (2008b). A three-stage implementation model for supply chain collaboration. Journal of Business Logistics 29(1), 93-112.

Fawcett, S. E., M. W. McCarter, A. M. Fawcett, G. S. Webb, and G. M. Magnan (2015). Why supply chain collaboration fails: the socio-structural view of resistance to relational strategies. Supply Chain Management: An International Journal 20(6), 648-663.

Firth, D. (1993). Bias reduction of maximum likelihood estimates. Biometrika $\underline{\text { 80 }}$ (1), 27-38.

Frisk, M., M. Göthe-Lundgren, K. Jörnsten, and M. Rönnqvist (2010). Cost allocation in collaborative forest transportation. European Journal of Operational Research 205(2), 448-458.

Ganesan, S. (1994). Determinants of long-term orientation in buyer-seller relationships. The Journal of Marketing $\underline{58}(2), 1-19$.

Gastner, M. T. and M. Newman (2006). Optimal design of spatial distribution networks. Physical Review E 74(1), 016117.

Gibson, B. J., S. M. Rutner, and S. B. Keller (2002). Shipper-carrier partnership issues, rankings and satisfaction. International Journal of Physical Distribution $\underline{\text { \& Logistics Management }} \underline{32}$ (8), 669-681.

Goffin, K., F. Lemke, and M. Szwejczewski (2006). An exploratory study of 'close' supplier-manufacturer relationships. Journal of operations management $24(2)$, 189-209.

Greene, W. H. (2003). Econometric analysis. Pearson Education India.

Grigoriev, A., V. Jung, and D. Munten (2019a). Urban area tessellations: Are regular tessellations good for non-uniformly distributed demand? submitted to Journal of Urban Economics. 
Grigoriev, A., V. Jung, and L. Verdonck (2019b). Comprehensive gain sharing maximizing satisfaction in supply chain collaborations. submitted to $\underline{\text { International Journal of Shipping and Transport Logistics. }}$

Grunbaum, B. and G. C. Shephard (1977). Tilings by regular polygons. Mathematics Magazine 50(5), 227-247.

Guardiola, L. A., A. Meca, and J. Timmer (2007). Cooperation and profit allocation in distribution chains. Decision Support Systems 44(1), 17-27.

Hagedoorn, J. and B. Sadowski (1999). The transition from strategic technology alliances to mergers and acquisitions: an exploratory study. Journal of Management Studies $36(1), 87-107$.

Hallowell, M. R. and J. A. Gambatese (2009). Qualitative research: Application of the delphi method to cem research. Journal of Construction Engineering and Management $136(1), 99-107$.

Hann, M. (2013). Structure and Form in Design: Critical Ideas for Creative Practice. A\&C Black.

Hay, S. I., C. A. Guerra, A. J. Tatem, P. M. Atkinson, and R. W. Snow (2005). Urbanization, malaria transmission and disease burden in africa. Nature reviews. $\underline{\text { Microbiology }} \underline{3}(1), 81$.

Heikkilä, J. (2002). From supply to demand chain management: efficiency and customer satisfaction. Journal of Operations Management 20(6), 747-767.

Heinze, G. and M. Schemper (2002). A solution to the problem of separation in logistic regression. Statistics in Medicine 21(16), 2409-2419.

Horvath, L. (2001). Collaboration: the key to value creation in supply chain management. Supply Chain Management: An International Journal $\underline{6}(5), 205$ 207.

Hosmer Jr, D. W., S. Lemeshow, and R. X. Sturdivant (2013). Applied logistic regression. John Wiley \& Sons.

Jap, S. D. (2001). Pie sharing in complex collaboration contexts. Journal of Marketing Research $38(1), 86-99$.

Jedwab, R., L. Christiaensen, and M. Gindelsky (2015). Demography, urbanization and development: Rural push, urban pull and... urban push? The World Bank. 
Jharkharia, S. and R. Shankar (2005). It-enablement of supply chains: understanding the barriers. Journal of Enterprise Information Management $18(1)$, 11-27.

Johnson, R. B. (1997). Examining the validity structure of qualitative research. $\underline{\text { Education }} \underline{118}(2), 282$.

Jung, V., M. Peeters, and T. Vredeveld (2017). A framework for better evaluations of supply chain collaborations: Evidence from the dutch fast moving consumer goods industry. GSBE Research Memorandum (014).

Jung, V., M. Peeters, and T. Vredeveld (2018a). Disagreement on the gain sharing method in supply chain collaborations. Russian Management Journal 16(4), 537-562.

Jung, V., M. Peeters, and T. Vredeveld (2018b). Drivers and resistors for supply chain collaboration. In Operations Research Proceedings 2016, pp. 623-628. Springer.

Kalwani, M. U. and N. Narayandas (1995). Long-term manufacturer-supplier relationships: do they pay off for supplier firms? The Journal of Marketing $\underline{59}(1)$, 1-16.

Kampstra, R., J. Ashayeri, and J. Gattorna (2006). Realities of supply chain collaboration. The International Journal of Logistics Management $17(3), 312$ 330 .

Lambert, D. M., M. A. Emmelhainz, and J. T. Gardner (1996). Developing and implementing supply chain partnerships. The International Journal of Logistics Management $\underline{7}(2), 1-18$.

Lambert, D. M., M. A. Emmelhainz, and J. T. Gardner (1999). Building successful logistics partnerships. Journal of Business Logistics 20(1), 165-181.

Landeros, R., R. Reck, and R. E. Plank (1995). Maintaining buyer-supplier partnerships. Journal of Supply Chain Management 31(2), 2-12.

Leng, M. and M. Parlar (2005). Game theoretic applications in supply chain management: a review. INFOR: Information Systems and Operational $\underline{\text { Research }}$ 43(3), 187-220. 
Leng, M. and M. Parlar (2009). Allocation of cost savings in a three-level supply chain with demand information sharing: A cooperative-game approach. Operations Research 57(1), 200-213.

Levenberg, K. (1944). A method for the solution of certain non-linear problems in least squares. Quarterly of Applied Mathematics 2(2), 164-168.

Liu, P., Y. Wu, and N. Xu (2010). Allocating collaborative profit in less-thantruckload carrier alliance. Journal of Service Science and Management 3 (1), 143-149.

Liu, R. P., G. Rogers, S. Zhou, and J. Zic (2007). Topology control with hexagonal tessellation. International Journal of Sensor Networks 2(1-2), 91-98.

Loulou, R. and A. Kanudia (1999). Minimax regret strategies for greenhouse gas abatement: methodology and application. Operations Research Letters $25(5)$, 219-230.

Lozano, S., P. Moreno, B. Adenso-Díaz, and E. Algaba (2013). Cooperative game theory approach to allocating benefits of horizontal cooperation. European $\underline{\text { Journal of Operational Research } 229}$ (2), 444-452.

Ludden, T. M., S. L. Beal, and L. B. Sheiner (1994). Comparison of the akaike information criterion, the schwarz criterion and the $\mathrm{f}$ test as guides to model selection. Journal of Pharmacokinetics and Biopharmaceutics 22(5), 431-445.

Mallozzi, L., E. D'Amato, and P. M. Pardalos (2017). Spatial Interaction Models: Facility Location Using Game Theory, Volume 118. Springer.

Mantel, S. P., M. V. Tatikonda, and Y. Liao (2006). A behavioral study of supply manager decision-making: Factors influencing make versus buy evaluation. Journal of Operations Management $24(6), 822-838$.

Marquardt, D. W. (1963). An algorithm for least-squares estimation of nonlinear parameters. Journal of the Society for Industrial and Applied Mathematics 11(2), 431-441.

Mason, R., C. Lalwani, and R. Boughton (2007). Combining vertical and horizontal collaboration for transport optimisation. Supply Chain Management: An International Journal 12(3), 187-199. 
Mather, M. and M. K. Johnson (2000). Choice-supportive source monitoring: Do our decisions seem better to us as we age? Psychology and Aging 15(4), 596-606.

Matopoulos, A., M. Vlachopoulou, V. Manthou, and B. Manos (2007). A conceptual framework for supply chain collaboration: empirical evidence from the agri-food industry. Supply Chain Management: An International Journal 12(3), $177-186$.

Mausser, H. E. and M. Laguna (1999). A heuristic to minimax absolute regret for linear programs with interval objective function coefficients. European Journal of Operational Research $\underline{117}(1), 157-174$.

Mendes, A. B. and I. H. Themido (2004). Multi-outlet retail site location assessment. International Transactions in Operational Research 11(1), 1-18.

Min, H. and G. Zhou (2002). Supply chain modeling: past, present and future. Computers \& Industrial Engineering 43(1/2), 231-249.

Min, S., A. S. Roath, P. J. Daugherty, S. E. Genchev, H. Chen, A. D. Arndt, and R. Glenn Richey (2005). Supply chain collaboration: what's happening? The International Journal of Logistics Management 16(2), 237-256.

Mohr, J. and R. Spekman (1994). Characteristics of partnership success: partnership attributes, communication behavior, and conflict resolution techniques. $\underline{\text { Strategic Management Journal 15 }}$ 12), 135-152.

Morris, G., D. Clinton, and S. Rutherford (1811). Remarks of the commissioners for laying out streets and roads in the city of new york, under the act of april 3, 1807. Online at http://www. library. cornell. edu/Reps/DOCS/nyc1811. htm.

Moulin, H. (1991). Axioms of cooperative decision making. Number 15. Cambridge university press.

Mueller, C. W. and J. C. McCloskey (1990). Nurses' job satisfaction: a proposed measure. Nursing Research 39(2), 113-117.

Mühlenfeld, H.-U. (2005). Differences between 'talking about' and 'admitting' sensitive behaviour in anonymous and non-anonymous web-based interviews. Computers in Human Behavior 21(6), 993-1003.

Nanda, A. and P. J. Williamson (1995). Corporate restructuring: How joint ventures can help ease the pain. INSEAD Paris. 
Narula, S. C. and U. I. Ogbu (1979). An hierarchal location-allocation problem. Omega $\underline{7}(2), 137-143$.

Nederhof, A. J. (1985). Methods of coping with social desirability bias: A review. European Journal of Social Psychology 15(3), 263-280.

Niederkofler, M. (1991). The evolution of strategic alliances: Opportunities for managerial influence. Journal of Business Venturing $\underline{6}$ (4), 237-257.

Noble, H. and J. Smith (2015). Issues of validity and reliability in qualitative research. Evidence-Based Nursing 18(2), 34-35.

OECD (2003). Delivering the goods - 21st century challenges to urban goods transport. https://www.itf-oecd.org/sites/default/files/docs/ 03deliveringgoods.pdf. Accessed:2017-11-30.

Oliver, R. L. (2014). Satisfaction: A behavioral perspective on the consumer. Routledge.

Özener, O. Ö. and Ö. Ergun (2008). Allocating costs in a collaborative transportation procurement network. Transportation Science 42(2), 146-165.

Patel, A. and R. Joshi (2009). Energy conservation for wireless mobile ad hoc networks using hexagonal gaf protocol. In Proceedings of the 12th International Conference on networking, VLSI and signal processing, pp. 29-34.

Perry, M. and A. S. Sohal (2001). Effective quick response practices in a supply chain partnership-an australian case study. International Journal of Operations $\underline{\text { \& Production Management } 21(5 / 6), 840-854 .}$

Qu, S. Q. and J. Dumay (2011). The qualitative research interview. Qualitative $\underline{\text { Research in Accounting \& Management }}$ 8(3), 238-264.

Reniers, G., W. Dullaert, and L. Visser (2010). Empirically based development of a framework for advancing and stimulating collaboration in the chemical industry (asc): creating sustainable chemical industrial parks. Journal of Cleaner $\underline{\text { Production }}$ 18$(16 / 17), 1587-1597$.

Richey Jr, G. R., H. Chen, R. Upreti, S. E. Fawcett, and F. G. Adams (2009). The moderating role of barriers on the relationship between drivers to supply chain integration and firm performance. International Journal of Physical Distribution \& Logistics Management $\underline{39}$ (10), 826-840. 
Richey Jr, R. G., A. S. Roath, J. M. Whipple, and S. E. Fawcett (2010). Exploring a governance theory of supply chain management: barriers and facilitators to integration. Journal of Business Logistics 31(1), 237-256.

Riggin, L. J., P. G. Grasso, and M. L. Westcott (1992). A framework for evaluating housing and community development partnership projects. $\underline{\text { Public }}$

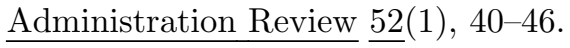

Ryu, I., S. So, and C. Koo (2009). The role of partnership in supply chain performance. Industrial Management \& Data Systems $\underline{109}$ (4), 496-514.

Sabath, R. E. and J. Fontanella (2002). The unfulfilled promise of supply chain collaboration. Supply Chain Management Review 6(4), 24-29.

Samaha, S. A., R. W. Palmatier, and R. P. Dant (2011). Poisoning relationships: Perceived unfairness in channels of distribution. Journal of Marketing $75(3)$, 99-117.

Schenk, D. H. (2011). Exploiting the salience bias in designing taxes. Yale Journal on Regulation 28 2 (2), 253-312.

Schmeidler, D. (1969). The nucleolus of a characteristic function game. SIAM Journal on Applied Mathematics $17(6), 1163-1170$.

Schmoltzi, C. and C. M. Wallenburg (2011). Horizontal cooperations between logistics service providers: motives, structure, performance. International Journal of Physical Distribution \& Logistics Management $\underline{41}$ (6), 552-575.

Schultz, G. P. (1970). The logic of health care facility planning. Socio-Economic Planning Sciences $\underline{4}(3), 383-393$.

Schwarz, G. et al. (1978). Estimating the dimension of a model. The Annals of $\underline{\text { Statistics }} \underline{6}(2), 461-464$.

Shapley, L. S. (1952). Notes on the n-person game, III: Some variants of the von Neumann-Morgenstern definition of solution. Rand Corporation.

Shapley, L. S. (1953). A value for n-person games. Contributions to the Theory of Games 2 (28), 307-317.

Shaw, K., R. Shankar, S. S. Yadav, and L. S. Thakur (2012). Supplier selection using fuzzy ahp and fuzzy multi-objective linear programming for developing low carbon supply chain. Expert Systems with Applications 39(9), 8182-8192. 
Sherman, S. (1992). Are strategic alliances working? http://archive.fortune . com/magazines/fortune/fortune_archive/1992/09/21/76881/index.htm. Accessed: 2017-11-30.

Simatupang, T. M. and R. Sridharan (2002). The collaborative supply chain. The $\underline{\text { International Journal of Logistics Management }}$ 13(1), 15-30.

Simatupang, T. M. and R. Sridharan (2004). Benchmarking supply chain collaboration: an empirical study. Benchmarking: An International Journal 11(5), 484-503.

Simon, H. A. (1979). Rational decision making in business organizations. The American Economic Review 69(4), 493-513.

Sin, C.-Y. and H. White (1996). Information criteria for selecting possibly misspecified parametric models. Journal of Econometrics 71(12), 207-225.

Soll, J. B., K. L. Milkman, and J. W. Payne (2014). A user's guide to debiasing. Wiley Blackwell Handbook of Judgment and Decision Making.

Stank, T. P., S. B. Keller, and P. J. Daugherty (2001). Supply chain collaboration and logistical service performance. Journal of Business logistics 22(1), 29-48.

Statista, T. S. P. (2018). https://www.statista.com/statistics/270860/ urbanization-by-continent/. Accessed: 2018-11-09.

Steinfield, C., M. L. Markus, and R. T. Wigand (2011). Through a glass clearly: standards, architecture, and process transparency in global supply chains. Journal of Management Information Systems 28(2), 75-108.

Sterman, J. D. (1989). Modeling managerial behavior: Misperceptions of feedback in a dynamic decision making experiment. Management Science 35(3), 321-339.

Stojmenovic, I. (1997). Honeycomb networks: Topological properties and communication algorithms. IEEE Transactions on parallel and distributed systems $\underline{8}(10), 1036-1042$.

Tijs, S. H. and T. S. Driessen (1986). Game theory and cost allocation problems. Management Science 32(8), 1015-1028.

Tokar, T. (2010). Behavioural research in logistics and supply chain management. The International Journal of Logistics Management 21(1), 89-103. 
Traynor, M. and B. Wade (1993). The development of a measure of job satisfaction for use in monitoring the morale of community nurses in four trusts. Journal of Advanced Nursing 18(1), 127-136.

Vanovermeire, C., K. Sörensen, A. Van Breedam, B. Vannieuwenhuyse, and S. Verstrepen (2014). Horizontal logistics collaboration: decreasing costs through flexibility and an adequate cost allocation strategy. International Journal of Logistics Research and Applications 17(4), 339-355.

Verdonck, L. (2017). Collaborative logistics from the perspective of freight transport companies. Ph. D. thesis.

Verdonck, L., P. Beullens, A. Caris, K. Ramaekers, and G. K. Janssens (2016). Analysis of collaborative savings and cost allocation techniques for the cooperative carrier facility location problem. Journal of the Operational Research Society $\underline{67}(6), 853-871$.

Walker, H., L. Di Sisto, and D. McBain (2008). Drivers and barriers to environmental supply chain management practices: Lessons from the public and private sectors. Journal of Purchasing and Supply Management 14(1), 69-85.

Warner, S. L. (1965). Randomized response: A survey technique for eliminating evasive answer bias. Journal of the American Statistical Association 60(309), 63-69.

Watson, R. (1973). Semi-regular tessellations. The Mathematical Gazette $57(401)$, $186-188$.

Whipple, J. M. and R. Frankel (2000). Strategic alliance success factors. Journal of Supply Chain Management 36(2), 21-28.

Wilkinson, D. and P. Birmingham (2003). Using research instruments: A guide for researchers. Psychology Press.

Winkler, H., D. Jodin, and M. Toferer (2015). Biomimetics applied to factory layout planning: Honeycombs as bio-inspiration to reduce internal transport costs in factories. Conference: XXI Triennial International conference on Material handling, constructions and logistics, At Vienna.

Wolfram, M. (2017). Tessellation. http://mathworld.wolfram.com/ Tessellation.html. Accessed:2017-11-28. 
Yan, L., G. Kuang, Q. Zhang, X. S. Shang, P. N. Liu, and N. Lin (2017). Selfassembly of a binodal metal-organic framework exhibiting a demi-regular lattice. Faraday Discussions.

Yin, R. K. (2013). Case study research: Design and methods. Sage publications.

Zineldin, M. and T. Bredenlöw (2003). Strategic alliance: synergies and challenges: A case of strategic outsourcing relationship "sour". International Journal of Physical Distribution \& Logistics Management 33(5), 449-464.

Zineldin, M., H. Fujimoto, Y. Li, H. Kassean, V. Vasicheva, and W. F. Yu (2015). Why do both marriages and strategic alliances have over $50 \%$ failure rate? a study of relationship quality of strategic alliances in china, japan and mauritius. $\underline{\text { International Journal of Strategic Business Alliances }} \underline{4}(1), 1-23$. 


\section{Valorization}

Knowledge valorization refers to the process of translating scientific knowledge into social benefits. In this addendum, I outline the knowledge valorization of the results obtained during my $\mathrm{PhD}$.

The research presented in this thesis deals with selected supply chain collaboration (SCC) challenges: the identification and understanding of relevant drivers and resistors for launching an SCC, gain sharing and the selection of a suitable coalition partner. In addition, ways to overcome these SCC challenges are proposed. In this thesis, the majority of the research findings are based on data from the Dutch fast moving consumer goods (FMCG) industry. As a result, the knowledge valorization for companies in a supply chain as a target group has already been described in each chapter itself. In the following, for each chapter the knowledge valorization is again briefly outlined. This is followed by a short overview about other possible target groups of this research.

Chapter 2 discusses the challenge of identifying and understanding relevant drivers and resistors for launching SCCs. To eliminate a barrier before it leads to the breakdown of the SCC, it is essential for the collaborative party to be aware of this barrier. In Chapter 2, a comprehensive, structured and consistent framework including all potential drivers and resistors for launching an SCC for a broad range of SCC types, perspectives and industries has been developed. This framework supports parties in a supply chain to more holistically evaluate the considered SCC and thus helps to identify collaborations with high failure potential. As a result, the probability of sustainable SCCs, which are nowadays very important for every 
company in a supply chain to be competitive on the dynamic market, is increased. The introduced framework provides to all collaborative parties an extensive checklist of drivers and resistors, which should be evaluated on their importance for the considered collaboration before launching the SCC. The framework further supports supply chain parties in their decision whether to launch a specific SCC or not. Only if the factors, which have a positive influence on SCC outweigh those with a negative influence, the considered SCC is advisable to launch.

Chapters 3 and 4 investigate the challenge of fairly sharing the coalition gain between the collaborative parties. If one party is not satisfied with its received gain share or if the party has the feeling that it is treated unfairly, future SCC are less likely to occur. As a result, in order to increase the probability of sustainable SCCs, the fair allocation of the coalition gain, which is also perceived as fair by all collaborative parties, is necessary.

Chapter 3 investigates the parties' acceptance of selected gain sharing methods. In order to be able to explain the cause of the acceptance or rejection of a gain sharing method, the influence of behavioral aspects, namely the availability of information and cognitive biases, on the party's acceptance of a gain sharing method has been observed. The incorporation of behavioral decision-making aspects ensure the practical validity of the results, since it is taken into account that human beings do not think rational. For a long time, the predominant assumption in economics was that human beings are rational thinking agents, which implies that decisions are made in a rational and consistent way. However, human beings are bounded due to limitations in available time, information and cognitive capabilities. We tend to rely on heuristics or cognitive biases to deal with complex problems. The main result of this chapter is that due to a different influence of information availability and diverse cognitive biases, no gain sharing method is accepted by all parties involved. As a result of the different influence of available information, one practical implication is to not provide the same amount of information to all collaborative parties, since this would lead to no preferred gain sharing method. Therefore, to each party separately an individual amount of information should be provided. Moreover, an influence of diverse cognitive biases could be identified. Cognitive biases make decisions more unpredictable, since decisions are not made in a rational and consistent way. In order to make the decisions more predictable, debiasing-techniques should be applied in practice. One example for a debiasing-technique is to provide all relevant information packaged 
in an intuitively comprehensible and compelling format. A suggestion in regard to gain sharing would be to show a figure which provides information about the allocation of the coalition gain.

Chapter 4 proposes a comprehensive, fair and simple gain sharing system, which focuses on the maximization of the parties' satisfaction. By focusing on the maximization of the parties' satisfaction, the satisfaction of all collaborative parties with the coalition gain should be guaranteed and as a result the probability of having sustainable SCCs should be achieved. The proposed gain sharing system can be easily applied into practice, since it provides all key characteristics, which are appreciated in practice: mathematical simplicity, applicability and transparency. Furthermore, the gain sharing system only requires limited input data in order to provide robust output for the gain sharing decision. In addition, the gain sharing system includes the evaluation of important key performance indicators such as the optimal gain share allocation and the fairness properties, which supports managers to evaluate the performance of the gain sharing system.

In Chapter 5 an approach which supports parties in the partner selection process is proposed. Special attention is paid to the geographical coverage, which is an important aspects for companies especially in the transport and logistics industry. However, geographical coverage cannot be achieved alone, but collaborations between parties from the same supply chain level are needed. The proposed approach supports parties in their partner selection process by suggesting where the coalition partner's facilities should be preferably located in order to achieve a significant geographical coverage. Simultaneously, the approach might reduce the transport costs in the horizontal collaboration, which represents another huge challenge in the transport industry, by assigning the demand to the facilities/collaborative parties in a cost efficient manner. In addition, the approach ensures, that the geographical markets of the parties do not overlap, which avoids the competitive pressure resulting out of overlaps in the customer base.

In this thesis, the focus has been on collaborations between parties in a supply chain. However, collaborations do not just appear between parties in a supply chain, but also all around us in e.g. our everyday relationships or at work. As a result, the proposed approaches can also be applied, in an adjusted version, to other collaborations. In the following, some examples are provided in which collaborations are essential. 
One example is the collaboration between researchers. The majority of researchers collaborate at a certain point in time to do research and/or to publish a paper together. Besides researchers, also students have to collaborate with each other at a certain point in time during their education. If students have to sit down together to exchange their ideas to solve a problem, the students are collaborating with each other. Recently, so-called real-life escape rooms have become popular. In these real-life escape rooms a group of people is locked in a room, where they have to solve puzzles in order to escape the room in a specific time frame. In order to successfully escape the room, the collaboration between the players is essential.

As a whole, I can summarize, that the research I performed during my $\mathrm{PhD}$ has the focus on collaborations between parties in a supply chain. However, since collaborations happen every day to multiple persons, the research findings are not limited to one area. 


\section{Nederlandse Samenvatting}

In de loop van de laatste decennia zijn bedrijven de voordelen beginnen inzien van het opzetten van een Supply Chain-samenwerking. Verschillende uitdagingen - zoals schaarse middelen, toegenomen concurrentie tussen de organisaties en hogere verwachtingen van de consument - hebben de bedrijven gedwongen om buiten hun organisatiegrenzen te zoeken naar partijen met wie ze kunnen samenwerken. Verschillende onderzoekers hebben de duurzame concurrentievoordelen geschetst die kunnen worden bereikt door samenwerking in de toeleveringsketen; zoals kostenbesparingen, cyclustijdverkorting en een verbeterde dienstverlening.

Ondanks dat Supply Chain-samenwerkingsverbanden aanzienlijke voordelen hebben, brengt deze samenwerking ook uitdagingen met zich mee. In dit proefschrift worden drie samenwerkings-uitdagingen, alsook benaderingen om deze uitgagingen aan te pakken, kritisch onderzocht. Ten eerste is onder de loep genomen hoe moeilijk het is om de drijfveren en de belemmeringen van het opstarten van een dergelijke samenwerking, te identificeren en te begrijpen. Ten tweede wordt de uitdaging van het verdelen van de winst besproken. Ten derde wordt een aanpak geschetst hoe de juiste coalitiepartner te vinden op basis van geografische karakteristieken.

Hoofdstuk 2 van dit proefschrift onderzoekt hoe moeilijk het is om alle potentiële drivers en hindernissen voor het opstarten van een Supply Chain-samenwerking te identificeren en te begrijpen. Om een belemmering te elimineren voordat deze leidt tot het uiteenvallen van de samenwerking, moet men zich immers bewust zijn van dit obstakel. In dit hoofdstuk wordt een uitgebreid kader voorgesteld 
dat alle drijfveren en drempels omvat voor het opstarten van een samenwerking voor een breed scala van samenwerkingsstructuren, perspectieven en industrieën in de Supply Chain. Het concept is versterkt door een duidelijk onderscheid tussen de vier categorieën drijfveren en hindernissen, en consistente definities en verklaringen van de invloed van deze categorieën op een samenwerking in de Supply Chain. Dit verbetert sterk de evaluatie door de samenwerkende partijen van een mogelijke collaboratie in de toeleveringsketen.

Hoofdstukken 3 en 4 bespreken de uitdagingen van het verdelen van de winst van de coalitie over de samenwerkende partijen. Voor een duurzame samenwerking in de toeleveringsketen is het noodzakelijk dat alle samenwerkende partijen tevreden zijn met hun toegewezen aandeel en dat ze het gevoel hebben dat ze een eerlijk deel van de coalitiewinst ontvangen.

In hoofdstuk 3 wordt gekeken hoe, in een verticale samenwerking met drie echelon-toeleveringsketens binnen de Nederlandse Fast Moving Consumer Goods industrie, de geselecteerde winstdeel-methodes worden aanvaard. Er wordt ook een verklaring gezocht waarom een winstdeel-methode al dan niet wordt aanvaard. Hierbij wordt de invloed van twee gedragsaspecten van beslissingen beschouwd, namelijk de beschikbaarheid van informatie enerzijds en cognitieve vooroordelen anderzijds. Dit onderzoek heeft twee belangrijke bevindingen aan het licht gebracht. Ten eerste blijken de beschikbare informatie en cognitieve vooroordelen, de aanvaarding van de winstdeel-methode door de verschillende partijen te beïnvloeden. Ten tweede wordt elke partner individueel beïnvloed door de beschikbaarheid aan informatie en door de verschillende cognitieve vooroordelen. Als gevolg van de individuele invloed van de beschikbaarheid van informatie en de verschillende cognitieve vooroordelen, wordt geen enkele toewijzingsmethode geaccepteerd door alle samenwerkende partijen.

Hoofdstuk 4 bouwt verder op de resultaten van Hoofdstuk 3. Er wordt een uitgebreid, robuust en eenvoudig winstdeelsysteem geïntroduceerd dat de aanvaarding en de tevredenheid van alle samenwerkende partijen garandeert. Door te focussen op het maximaliseren van de tevredenheid van de partijen wordt de kans op een duurzame Supply Chain-samenwerking vergroot. Het voorgestelde winstdeelsysteem vertoont alle belangrijke kenmerken die in de praktijk worden gewaardeerd, zoals wiskundige eenvoud, toepasbaarheid en transparantie. Bovendien is vast- 
gesteld dat het winstdelingssysteem een eerlijke verdeling van winsten oplevert, wat kan leiden tot duurzame samenwerkingsverbanden in de Supply Chain.

Hoofdstuk 5 onderzoekt de uitdaging om een geschikte coalitiepartner te vinden voor een horizontale samenwerking in de Supply Chain en dit specifiek, in de logistieke sector. Een grote uitdaging in deze sector is de geografische dekking. De gewenste geografische dekking kan niet door één partij alleen bereikt worden, maar enkel door samenwerking met Supply Chain partners van hetzelfde niveau. Daarom ligt de focus van het partnerselectieproces op deze geografische dekking. In hoofdstuk 5 wordt een aanpak voorgesteld die de partijen ondersteunt in hun partnerselectie-proces door te suggereren waar de faciliteit van de partner bij voorkeur moet worden geplaatst om een significante geografische dekking te bereiken. Tegelijkertijd vermindert deze aanpak eveneens de transportkosten een andere grote uitdaging in de logistieke sector. Tot slot wordt in deze strategie - aangezien de tesselaties een vlak zonder overlappingen bedekken - de concurrentiedruk die door zulke overlappingen in het klantenbestand kan ontstaan, vermeden. 



\section{Curriculum Vitae}

Verena Jung was born on March 26, 1990 in Aachen, Germany. She attended high school between 2000 and 2009 at St. Ursula Gymnasium in Aachen, where she received her Gymnasium diploma. Subsequently, Verena studied Business Administration at RWTH Aachen University, where she received her M.Sc. degree in January, 2015.

After graduation, Verena started a joint PhD program at the Department of Quantitative Economics at Maastricht University, under the supervision of Prof. dr. Alexander Grigoriev and Dr. Tjark Vredeveld, and at the Logistics Group at UHasselt, under the supervision of Prof. dr. Katrien Ramakers and Dr. Lotte Verdonck. The results of her research are presented in this thesis. During her PhD, Verena presented her work at various international conferences. Chapters of this book have been published or are currently under revision in international refereed academic journals. In 2018 Verena started working as a supply chain analyst at Medtronic in Heerlen. 




\section{ISBN 978-94-6380-825-5}

